\title{
WestVirginiaUniversity
}

THE RESEARCH REPOSITORY @ WVU

Graduate Theses, Dissertations, and Problem Reports

2005

\section{Investigation of relative humidity and induced-vortex effects on aircraft icing}

Egemen O. Ogretim

West Virginia University

Follow this and additional works at: https://researchrepository.wvu.edu/etd

\section{Recommended Citation}

Ogretim, Egemen O., "Investigation of relative humidity and induced-vortex effects on aircraft icing" (2005). Graduate Theses, Dissertations, and Problem Reports. 2704.

https://researchrepository.wvu.edu/etd/2704

This Dissertation is protected by copyright and/or related rights. It has been brought to you by the The Research Repository @ WVU with permission from the rights-holder(s). You are free to use this Dissertation in any way that is permitted by the copyright and related rights legislation that applies to your use. For other uses you must obtain permission from the rights-holder(s) directly, unless additional rights are indicated by a Creative Commons license in the record and/ or on the work itself. This Dissertation has been accepted for inclusion in WVU Graduate Theses, Dissertations, and Problem Reports collection by an authorized administrator of The Research Repository @ WVU.

For more information, please contact researchrepository@mail.wvu.edu. 


\title{
INVESTIGATION OF RELATIVE HUMIDITY AND INDUCED-VORTEX EFFECTS ON AIRCRAFT ICING
}

EGEMEN O. OGRETIM

\author{
Dissertation submitted to the \\ College of Engineering and Mineral Resources \\ at West Virginia University \\ in partial fulfillment of the requirements \\ for the degree of
}

Doctor of Philosophy

In

Aerospace Engineering

Wade W. Huebsch, Ph.D., Chair

David C. Lewellen, Ph.D.

Gary J. Morris, Ph.D.

John M. Kuhlman, Ph.D.

Rakesh K. Gupta, Ph.D.

Department of Mechanical and Aerospace Engineering

Morgantown, West Virginia

2006

Keywords: Aircraft Icing, Relative Humidity, Vortex Dynamics

Copyright 2006 Egemen O. Ogretim 


\section{ABSTRACT \\ INVESTIGATION OF RELATIVE HUMIDITY AND INDUCED-VORTEX EFFECTS ON AIRCRAFT ICING \\ EGEMEN O. OGRETIM}

Aircraft icing is an area of research that has drawn attention since the early times of powered flight at high altitudes. Since World War II, aircraft icing research has gained a great deal of momentum, and several branches of research have developed as a result. These branches include the experimental, analytical and computational methods. With the advent of high-speed computers, the computational methods are becoming the leading icing research area due to their low cost requirements. However, a significant hindrance is the lack of a complete understanding of the icing phenomena, which leads to discrepancies between the predictions and the experiments. In recent years, there have been efforts to improve this situation by accounting for several mechanisms within the computational models. These mechanisms include the droplet splash and re-impingement, water film dynamics, and different heat transfer mechanisms. In support of enhancing the understanding of the aircraft icing process, this Ph.D. study focuses on the relative humidity effects and the interaction of the induced vortices with the droplets and the surface water.

Currently the relative humidity effects are neglected in the icing prediction codes with the assumption that it can at best be a second-order effect. This $\mathrm{Ph}$. D. study looks at the conditions in which the relative humidity effects can pose significant impact on the accreted ice shape. It was seen that the flow around the airfoil suction surfaces and the vortices, which have low-pressure cores, shed from the existing ice shape are highly supersaturated. Therefore, the suction surfaces and the aft regions of the main ice shape are exposed to condensation/deposition due to relative humidity effects. The time scales involved in the relative humidity effects were also investigated by using a numerical droplet growth experiment. In the particular case considered in this study, the required time to re-establish equilibrium, i.e. recover saturation conditions, varied from 12 milliseconds for droplets with 1 micron diameter to 5 seconds for droplets with 20 micron diameter. In an actual flight scenario, the direct impingement region mostly overlaps with the stagnation region, where the local flow is subsaturated. However, the water mass carried by the water droplets is much higher compared to changes that can be triggered by subsaturation. Therefore, it can be concluded that the collection efficiency in the direct impingement region will have negligible change despite the evaporation due to subsaturation. So, the relative humidity can at most be a secondary effect in the direct impingement region. However, exposure to supersaturated air for long periods can lead to localized frost growth beyond the direct impingement region. For a particular case considered in this study, it was found that there is a potential for the growth of extra ice roughness up to $0.4 \mathrm{~mm}$ over a 30 minute period.

As far as the induced vortex phenomena, no past work has been done, because the ice accretion prediction codes are based on potential flow formulation, which ignores the viscous effects. Therefore, 
this Ph.D. study is a first approach to show the existence of these mechanisms as potential contributors to the ice growth over the airframe. A Lagrangian particle tracking was performed to investigate the interaction of the droplets with the unsteady vortex shedding from the ice surface. It was seen that the vortices shed from ice surface can alter the trajectory paths of the droplets and change the ice growth process. The altered paths result in impingement on areas that are beyond the direct impingement region. For the particular case considered in this study, the percentage of the impingements in the aft region of the main ice shape varied up to $6.5 \%$ of the number of impingements in the frontal area of the main ice shape. For another case, the resultant water layer thickness due to the extra impingements over a 7 minute period was calculated as $0.24 \mathrm{~mm}$. It was also found in this study that the strength of the shed vortices increases with the flow Reynolds number, and therefore they become strong enough to affect the paths of larger droplets. 


\section{ACKNOWLEDGMENTS}

Address to the orphan:

The summer of 2002 was a fresh start for me in many respects: graduate study for $\mathrm{PhD}$ in aerospace, marriage, and deep scientific-philosophic and religious quests in my personal world. Now, I am in 2006. I am almost done with my PhD, have a baby boy, and hoping to close in on some conclusions in my scientific-philosophic and religious quests. All these state the end of a period in my life, and a start for the next one.

Everybody passes through these stages with some variations in depth and width. If I were to be asked at what depth and width I was, I would say I am an inch deep but worldwide. But, what good is that, if there is no fruit yielding from the tree?

It is only the recent months of my life that the tree of my personality yielded some fruits related to my inner world. I have articles that appeared in a quarterly magazine that is published worldwide, and have two more waiting to be approved for publishing; still working on another one. All stemming from the quests that I went through in the past years of my life during my $\mathrm{PhD}$. I consider these articles as the addresses that I made myself, which I will listen to in the future. By referring to the future, I mean and hope the near future, when I need advice from a close person that knows the turbulence inside.

Address of the orphan:

This $\mathrm{PhD}$ work has been done mainly under high pressure due to the extremely complicated character of the pressure Poisson equation and its unusual interaction with its boundary conditions. I would like to thank Dr. Huebsch for his patience and help during those times of hardship. With his help, I not only developed my skills of research but also grew in my teaching skills with the teaching opportunities he opened. I would like to thank him in advance for allowing me some time to be a good graduate student and a good family man at the same time.

I would like to recognize my committee members for the sacrifice they made from their valuable time to help me complete my Ph.D. research. Special thanks go to Dr. Lewellen for his availability and guidance during my studies.

My officemates who had to listen to the same movies, A Beautiful Mind and October Sky, over and over, also deserve recognition. I would like to thank them for being nice people to accommodate me in their warm atmosphere. Ibrahim, Zeynep, Cem, Jun Li, Raju, Christian, Andrew, Craig, Han Zhou, and 
Aaron: thank you. At this point, I would like to double-thank Aaron for his collaboration in the neural network research and for being a good comrade on the search for ultimate formulation for the fluid flow that would incorporate relativity and quantum theories.

Pressure solver, neural net, relativity: the three phrases that my life friend had to memorize due to my obsessive depressive relation with them. Thankfully, she was patient enough to bear with me. I would like to thank her for being my wife and being a good mother to our son as she is doing her $\mathrm{PhD}$ at the same time.

And my mother, who is my first teacher, deserves recognition for her continued support in the endeavors in my life and for her help by staying with us to take care of our baby as we, the parents, go to the university.

Et, la damme qui m'a invité au monde. Sache que ce n'est pas facile du tout à entrer la vie ici. Mais, le ciel n'a pas de sens sans le monde, non plus. Tu as une position remarkable dans ma vie.

And You, the One who guided me when I was not aware of anything, who inspired me with love and new ideas, the One who never gave up even if I gave up, who never turned away even if I turned away; thank You. 


\section{TABLE OF CONTENTS}

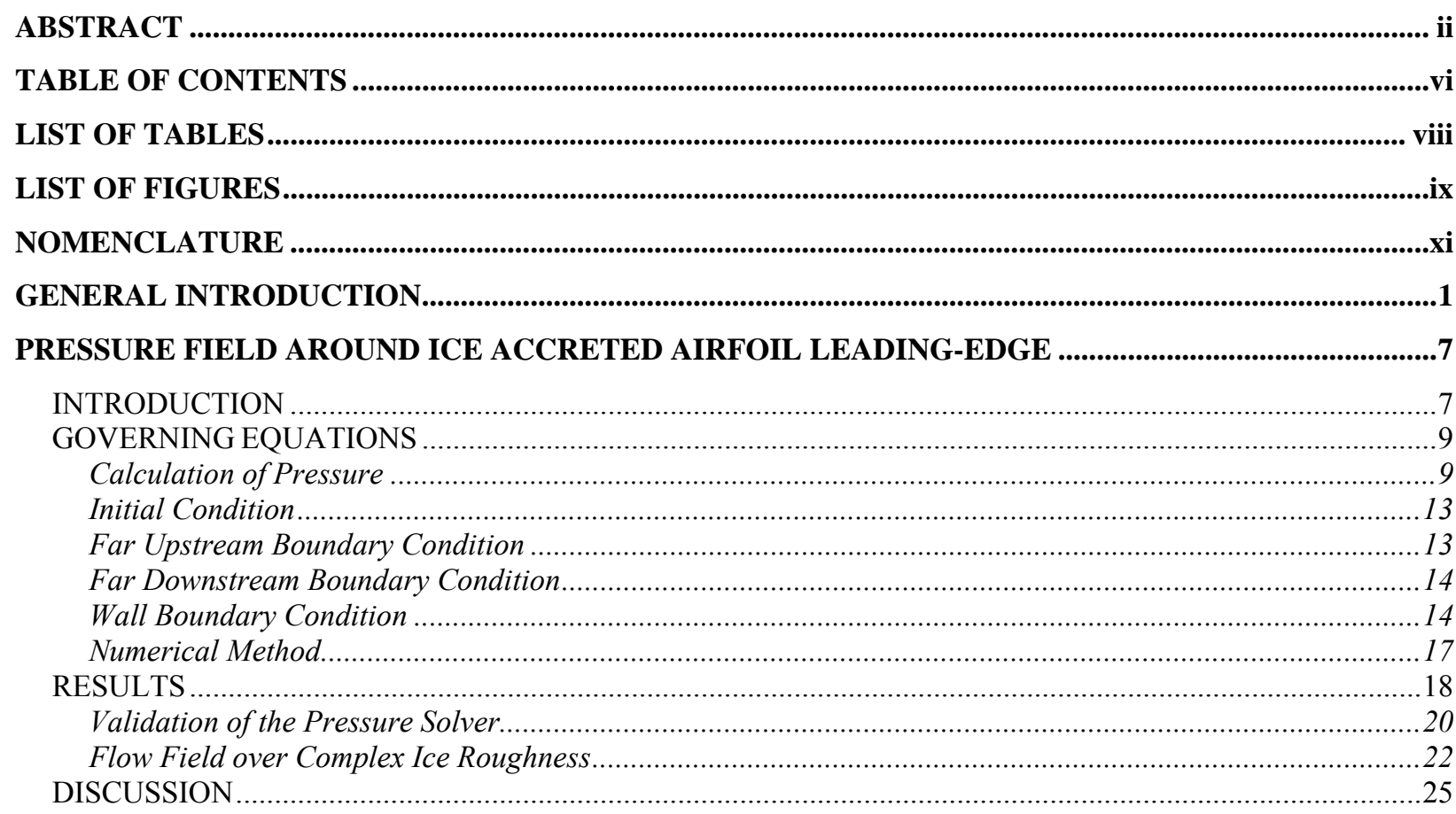

POTENTIAL OF LOCAL RELATIVE HUMIDITY TO AFFECT AIRCRAFT ICING...................................26

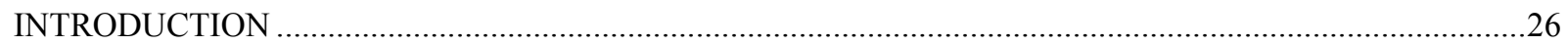

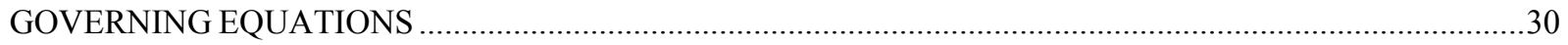

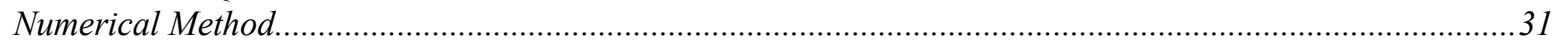

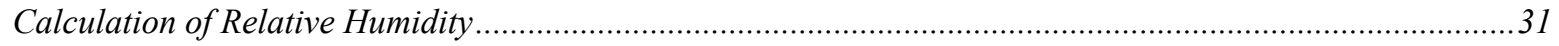

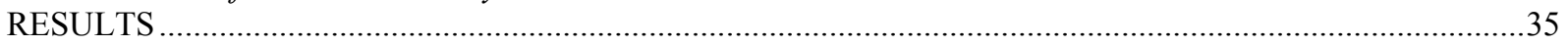

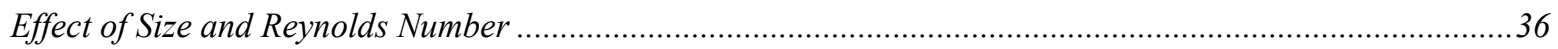

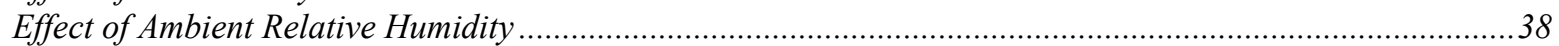

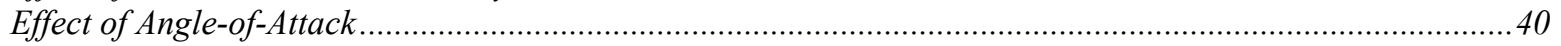

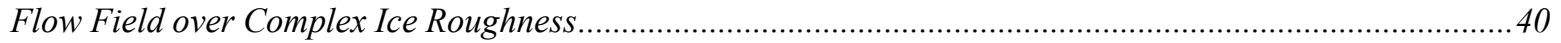

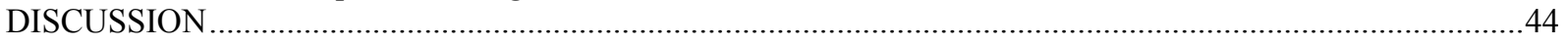

MECHANISMS FOR DOWNSTREAM ICE GROWTH ……...........................................................................46

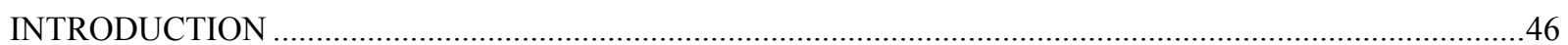

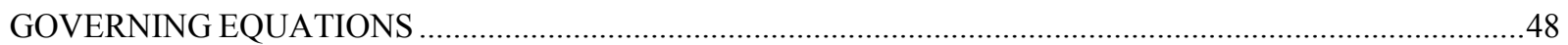

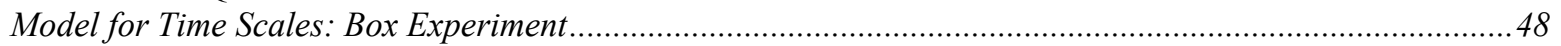

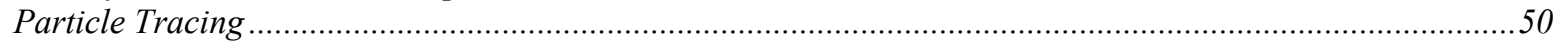

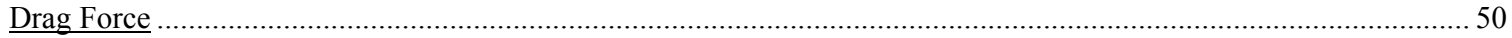

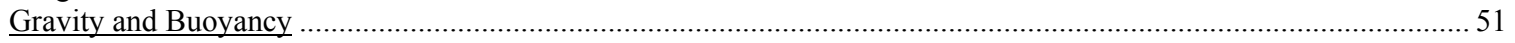

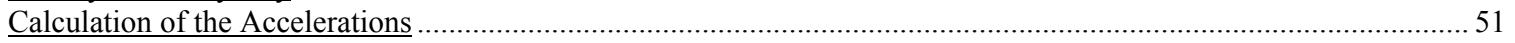

Numerical Approach for the Local Values........................................................................................................ 51

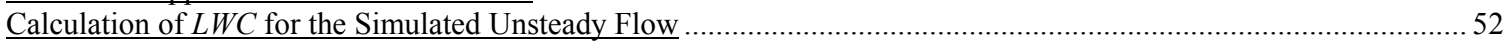

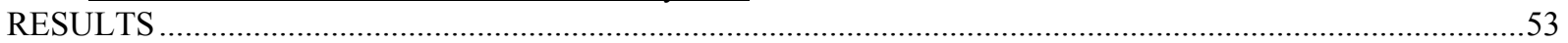

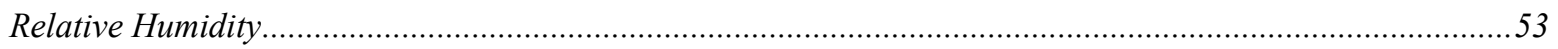

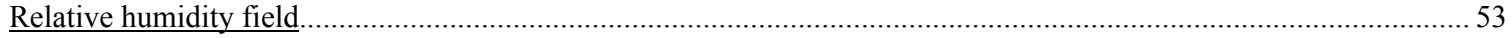

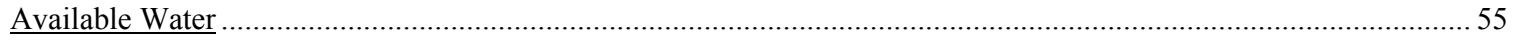

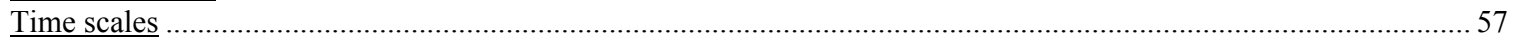

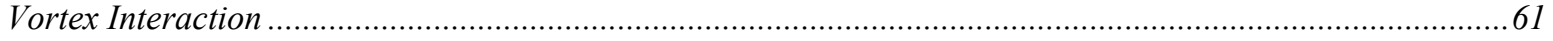

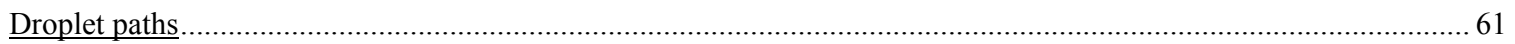

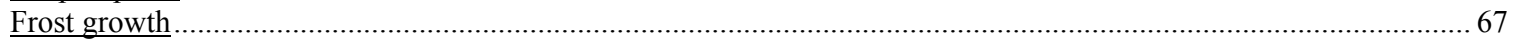




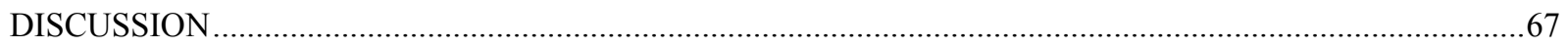

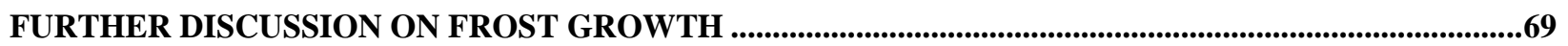

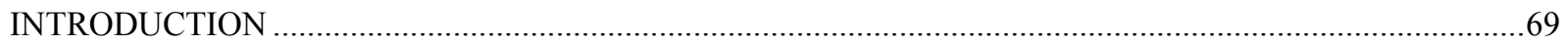

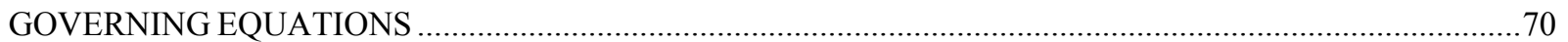

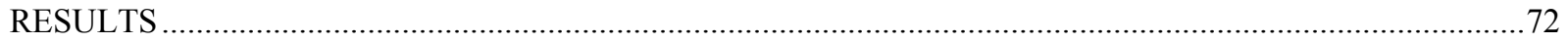

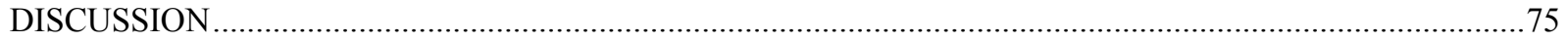

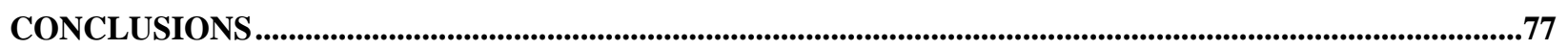

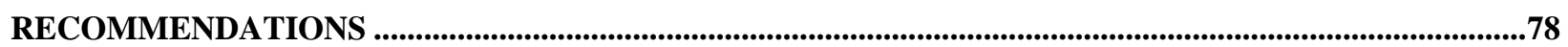

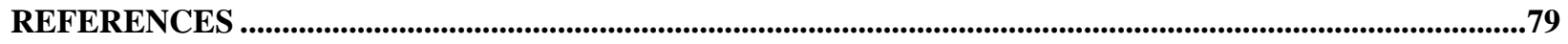

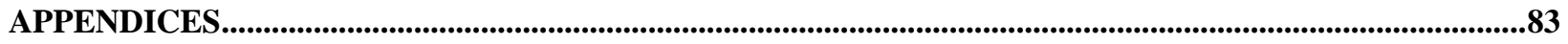

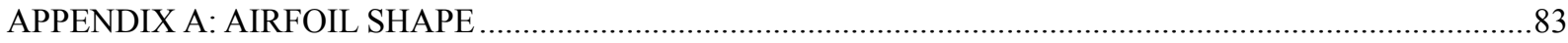

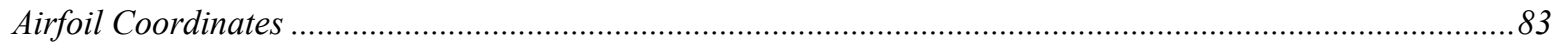

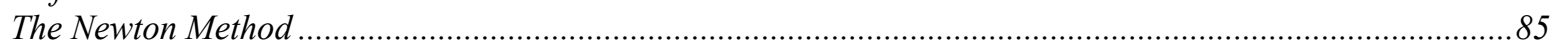

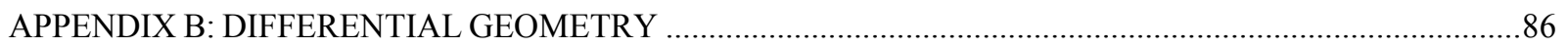

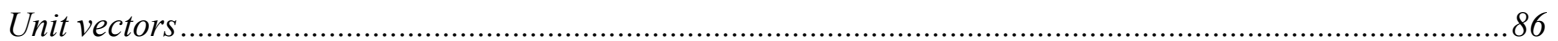

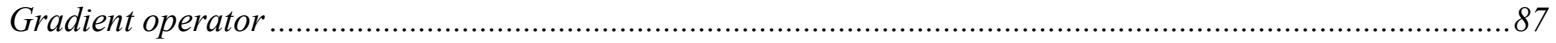

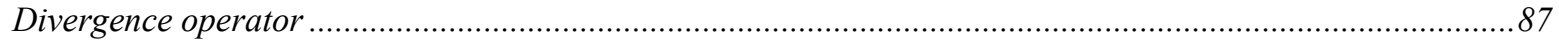

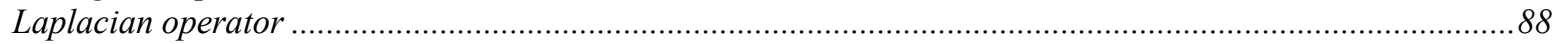

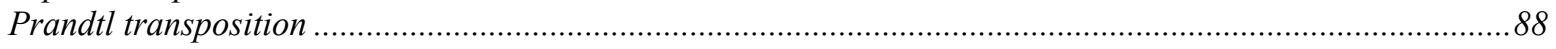

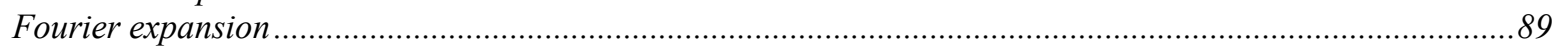

APPENDIX C: DERIVATION OF THE PRESSURE POISSON EQUATION …….........................................90

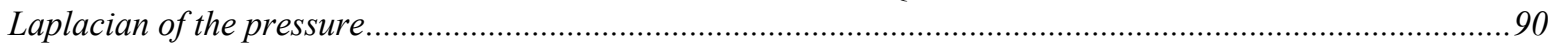

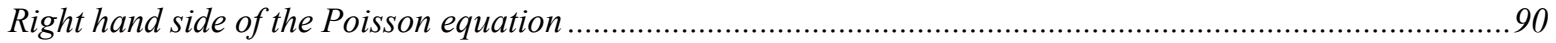

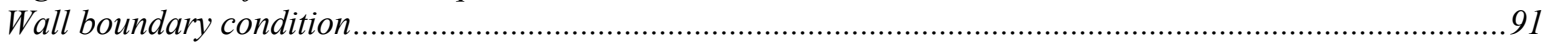

APPENDIX D: VELOCITY AND ACCELERATION VECTORS ................................................................95

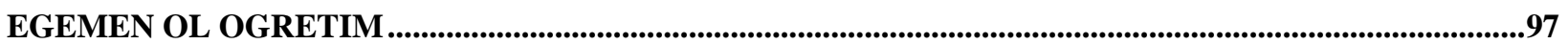




\section{LIST OF TABLES}

Table 1. The number of frames used to determine the separation between the neighboring droplets and the

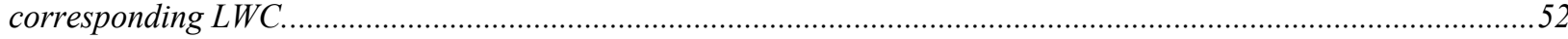

Table 2. Statistics of the interaction of the unsteady vortex field with the droplet paths........................................66 


\section{LIST OF FIGURES}

Figure 1. NACA 0012 leading-edge and the ice shape (I-1998-000092). __ 9

Figure 2. Geometrical depiction of the transformations.

Figure 3. Parabola and the ice shape (I-1998-000092) in the transformed plane. ___ 12

Figure 4. Depiction of the staggered grid approach.

Figure 5. Overview and a zoom in of the mesh over the ice shape (I-1998-000092).

Figure 6. A close up of the mesh around the ice shape (I-1998-000092). (x3500 Figure 5-a)_ 20

Figure 7. Non-dimensional pressure variation on the analytic hump. ___ 21

Figure 8. Non-dimensional pressure over a roughness element on a NACA 0015 airfoil, Beierle et al. [22]. __ 21

Figure 9. Pressure coefficient on a NACA 0021 airfoil upper surface. Ref [23]. __ 23

Figure 10. Calculated pressure coefficient distribution on a NACA 0021 leading-edge. ___ 23

Figure 11. Pressure field in an unsteady flow around experimental rime ice shape ${ }^{13}$ around the NACA 0012. 24

Figure 12. Pressure field in an unsteady flow around experimental glaze ice shape ${ }^{13}$ around the NACA 0012. 24

Figure 13. Experimental ice accretion on an airfoil and the corresponding LEWICE prediction, Ref. [13]. ___ 27

Figure 14. NACA 0012 leading-edge and the experimental glaze ice shape ${ }^{13}$. 29

Figure 15. Comparison of variation of relative humidity $(R H)$ as given by ideal gas and isentropic relations; values

are calculated for 21 inch chord length and $5000 \mathrm{~m}$ altitude in standard atmosphere; $\operatorname{Re}_{c}=1 \times 10^{6}$.

Figure 16. Variation of relative humidity $(R H)$ as a function of $C_{p}$ at $5000 \mathrm{~m}$ altitude in standard atmosphere;

$\operatorname{Re}_{c}=1.7 \times 10^{6}$.

Figure 17. Stream function contours around a hump and the comparison of the surface pressure on humps with

different sizes. $\operatorname{Re}_{\text {ler }}=100$.

Figure 18. A snapshot from the unsteady flow: stream function $(\boldsymbol{\psi})$ and the vorticiy $(\omega)$ contours around a simulated

roughness element. $R e_{\text {ler }}=5000$.

Figure 19. A snapshot from the unsteady flow: surface pressure and the pressure contours around a simulated roughness element. $\operatorname{Re}_{\text {ler }}=5000$.

Figure 20. Variation of maximum $R H_{w}$ and $R H_{\text {ice }}$ due to the ambient relative humidity.

Figure 21. $C_{p}$ variation over an analytic hump on upper surface at different angles of attack. $\operatorname{Re}_{l e r}=100$.

Figure 22. $C_{p}$ contours around ice accreted on NACA 0012 at $1^{\circ}$ angle-of-attack.

Figure 23. $\mathrm{RH}_{w}$ around ice accreted on NACA 0012 at $1^{\circ}$ angle-of-attack.____ 42

Figure 24. $R H_{\text {ice }}$ around ice accreted on NACA 0012 at $1^{\circ}$ angle-of-attack. 43

Figure 25. Variation of the vortex core relative humidity due to the ambient relative humidity.

Figure 26. Relative humidity field around NACA 0012 leading-edge with ice shape (AUG 1991 3-6-36) $)^{13} \cdot{ }_{-}$

Figure 27. Variation of excess water vapor mass as a function of relative humidity with respect to liquid water;

values are calculated for 5000m altitude in standard atmosphere.

Figure 28. Variation of excess water vapor mass as a function of Reynolds number; values are calculated at $C_{p}=-2$

for a chord length of 21 inches at 5000m altitude in standard altitude. 56

Figure 29. LWC history for different initial relative humidity levels.

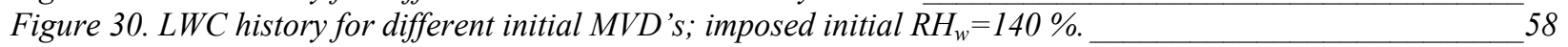

Figure 31. Change in roughness height due to relative humidity on the upper side of a NACA 0012 airfoil in flight with $\operatorname{Re}_{c}=1 \times 10^{6}$ at $5000 \mathrm{~m}$ standard altitude.

Figure 32. Change in roughness height due to relative humidity on the lower side of a NACA 0012 airfoil in flight with $\operatorname{Re}_{c}=1 \times 10^{6}$ at $5000 \mathrm{~m}$ standard altitude.

Figure 33. Stream function contours around a NACA 0012 airfoil with glaze ice showing the shed vortices. 61 Figure 34. Paths of the droplets (MVD=5 micron) that are released simultaneously in an unsteady flow around a NACA 0012 airfoil with glaze ice.

Figure 35. All droplet impingement paths (MVD=20 micron) in an unsteady flow around a NACA 0012 airfoil with glaze ice.

Figure 36. All droplet impingement paths (MVD=30 micron) in an unsteady flow around a NACA 0012 airfoil with

glaze ice.

Figure 37. All droplet impingement paths (MVD=40 micron) in an unsteady flow around a NACA 0012 airfoil with glaze ice.

Figure 38. All droplet impingement paths (MVD=50 micron) in an unsteady flow around a NACA 0012 airfoil with glaze ice.

Figure 39. The variation of the temperature within the frost subject to convective and conductive heat transfer. 
Figure 40. The variation of the relative humidity at the frost surface at different ambient pressures.

74

Figure 41. Effect of ambient pressure on the frost growth with various porosity levels at the surface that is kept at 0

${ }^{o} \mathrm{C}$.

Figure 42. Effect of frost surface temperature at sea level conditions with different porosity levels.

Figure 43. Change of time required to return to the saturation conditions as a function of initial droplet diameter. 76 


\section{NOMENCLATURE}

$a_{x}$ : Non-dimensional acceleration component in $\mathrm{x}$-direction

$a_{y}$ : Non-dimensional acceleration component in y-direction

$a_{\xi^{\prime}}:$ Acceleration component in $\xi^{\prime}$-direction

$a_{\eta^{\prime}}:$ Acceleration component in $\eta^{\prime}$-direction

$c$ : Chord length

$\mathrm{cm}$ : centimeter

$C_{p}$ : Pressure coefficient, $C_{p}=\left(P-P_{\infty}\right) / \frac{1}{2} \rho_{\text {air }} V_{\infty}^{2}$

$D$ : Diffusivity of water vapor in air $\left(\mathrm{m}^{2} / \mathrm{s}\right)$

DNS: Direct Numerical Simulation

$e$ : vapor pressure (milibar)

$h$ : height of the roughness element

$K$ : Thermal conductivity of water (J/m.s.K)

$k / c$ : ratio of roughness height to chord length

$L$ : Latent heat of vaporization for water $\left(2.5 \times 10^{6} \mathrm{~J} / \mathrm{kg}\right)$

ler: Leading-edge radius (m)

$L W C$ : Liquid water content ( $\mathrm{kg}$ of liquid water $/ \mathrm{m}^{3}$ of air)

$M V D$ : Median Volumetric Diameter (m)

Micron $(\mu)$ : Micrometer

$N$ : Number of droplets in $1 \mathrm{~m}^{3}$ of air

$P$ : Static (thermodynamic) pressure

$P_{v, \infty}:$ Vapor pressure in the ambient flow

$P_{v s, \infty}:$ Saturation vapor pressure in the ambient flow

$P_{v s, f s}:$ Saturation vapor pressure at the frost surface

$R_{v}$ : Specific gas constant for water vapor $(461 \mathrm{~J} / \mathrm{kg} . \mathrm{K})$ 
$R H_{w}$ : Relative humidity with respect to liquid water

$R H_{\text {ice }}$ : Relative humidity with respect to ice

$S$ : Cross-sectional area of a sphere $\left(\mathrm{m}^{2}\right)$

$s_{d}$ : Separation between two neighboring droplets (m)

$T$ : Static (thermodynamic) temperature

$V_{\text {inf }}$. Velocity of the approaching flow

$V W C$ : Vapor water content ( $\mathrm{kg}$ of water vapor $/ \mathrm{m}^{3}$ of air)

$v_{x}$ : Non-dimensional velocity component in $x$-direction

$v_{y}$ : Non-dimensional velocity component in $y$-direction

$v_{\xi^{\prime}}:$ Velocity component in $\xi^{\prime}$-direction

$v_{\eta^{\prime}}:$ Velocity component in $\eta^{\prime}$-direction

$w$ : mixing ratio of water vapor and dry air

$w_{\text {amb }}$ : mixing ratio in the ambient flow

$w_{\mathrm{fs}}$ : mixing ratio at the frost surface

$\rho_{\text {air }}$ : Density of dry air $\left(\mathrm{kg} / \mathrm{m}^{3}\right)$

$\rho_{v}$ : Density of water vapor $\left(\mathrm{kg} / \mathrm{m}^{3}\right)$

$\rho_{\text {water }}$ : Density of liquid water $\left(\mathrm{kg} / \mathrm{m}^{3}\right)$

$\gamma$ : Ratio of the specific heats 


\section{CHAPTER I}

\section{GENERAL INTRODUCTION}

Aircraft icing has been a major problem since the advent of powered flight. As a brief definition, icing is the ice accretion that occurs on various parts of the airframe due to favorable conditions for icing while on the ground or during the flight. Ice accretion affects the flight characteristics of an aircraft, degrades the performance of the power and communication units, and can cause loss in control of the aircraft $^{1}$. In some cases, ice build-up leads to crashes with fatalities, and in other cases, it causes severe damage to the aircraft. In either case, it poses a great threat to the flight safety and is a challenge to the aircraft manufacturers and aviation companies.

A brief explanation of ice accretion on aircraft surfaces is as follows ${ }^{2}$. When the aircraft enters a cloud that consists of supercooled liquid water droplets, these droplets impinge on the aircraft surfaces. Due to their metastable phase, the supercooled droplets freeze either completely or partially upon impact with the surface. The freezing fraction of the droplets is determined by the ambient conditions in the local atmosphere. If the droplets freeze completely upon impact, which occurs at temperatures well below the freezing point, they form an ice shape that is called rime ice. This kind of ice has a white color due to the air bubbles entrapped in the ice structure. Rime ice causes a thickened leading-edge with a rough surface on the exposed side of the airframe. If, on the other hand, the droplets freeze partially upon impact, they form an ice shape called glaze ice. Glaze ice has a transparent and pure crystal structure. This is because the gaps in the ice structure are filled with the remaining liquid water from the impinging droplets due to their interaction with the airflow. The glaze ice forms at temperatures slightly below the freezing point and is associated with formation of horn-shaped ice structures that grow into the flow.

In addition to the two primary ice accretion types explained above, i.e. rime ice and glaze ice, depending on where and when ice forms, there can be additional classifications. For example, intercycle ice $^{3}$ is the ice that accretes during the off-mode between two deicing cycles. Runback ice ${ }^{4}$ is the ice that accretes in the region that is beyond the anti/de-iced part of the airframe.

The ice accretion usually appears on the leading-edge of a surface that is exposed to droplet impingement. Therefore, the aircraft that are equipped with front loaded wings are more vulnerable to the ice accretion related deficiencies due to loss of a majority of their lift. Aside from loss of lift, ice accretion, even at a small amount, is linked to increased drag, and aberrations in the control and performance of the aircraft. Ice accretion increases the stall speed, too. Therefore, a safe speed in case of a clean wing may cause stall when ice is present on the wing surface. Most icing related accidents occur 
during the take-off/landing, holding, or approach phases of the flight ${ }^{5}$. These phases are the ones in which the aircraft flies at low power setting, hence low power for ice protection, and at low altitude, where more water density is expected. Also, due to the low speed setting in the mentioned phases of the flight, ice accretion can lead to early-stall, hence aircraft accidents.

To protect against icing on several components of the aircraft, there are ice protection systems. Mainly two types are in use: boots and hot air systems. The small aircraft can not afford a hot air system due to their low power range. Therefore they are equipped with inflatable boots that are activated by the pilot in case of an ice accretion (de-icing). Large passenger aircrafts, on the other hand, use hot air systems that either melt or evaporate the impinging droplets to the wing leading-edge surface (anti-icing). Aside from the anti-icing/de-icing mechanisms, the aircraft are also often sprayed with a fluid that prevents any kind of water accumulation on the wing surface during take-off.

The establishment of an icing research branch at the Glenn Research Center (GRC) of NASA took place in the early forties. The first research conducted then was mainly experimental, and some empirical results were extracted from those experiments. Namely, the heat transfer characteristics of a heated or an unheated surface were calculated to develop a more economical ice protection system. Today, the icing branch has a very-well equipped Icing Research Tunnel (IRT) that can simulate the icing cloud conditions in the air ${ }^{6}$. With this capability, IRT is used for experiments to improve the ice accretion modeling and prediction. IRT is also used for the verification of newly designed aircraft for flight into icing conditions.

Due to the high costs and time requirements for such a verification process, it is desirable to have a model to use as a prediction tool, given the aircraft geometry and the ambient atmospheric conditions. The first theoretical attempt for modeling the ice accretion process came from Messinger $^{2}$ shortly after the establishment of the GRC. The Messinger model is based on mass and energy conservation to predict the amount of ice accretion on the surface. However, there are several difficulties arising with the effort to model the mass and energy transport in the control volume of concern. The transport characteristics are extremely complicated due to the roughness-airflow interaction, coupled with possible pre-mature transition to turbulence. However, by making a series of engineering assumptions, the model proved to yield applicable results. Especially for rime ice cases, the results are very reliable. Due to the level of its success, the Messinger model has played a significant role in most icing research studies.

With the advent of the computer age, aeronautical sciences also enjoyed the computational speeds introduced by these machines. Along with aircraft design and aerodynamics, icing research also had its improvements through the implementation of the Messinger model into a computer code. Based on this model, several companies and organizations developed their own ice accretion prediction code, such as 
LEWICE $^{7}$ by NASA, and FENSAP-ICE ${ }^{8}$ by ONERA. The common property of these codes aside from the Messinger model is that they rely on the potential flow solution around the exposed wing to trace the droplet impingements.

The computer codes not only helped implement the icing model developed by Messinger but also helped investigate the validity of the algorithm. It was seen that the predictions made by the Messinger model worked fine under the rime ice conditions but did not do well for the glaze ice conditions, especially at large droplet sizes. The famous ATR 72 accident (due to build-up of an ice ridge behind the de-icing boots) in Roselawn, Indiana, and the Embraer EMB-120 accident (due to intercycle ice accretion) near Detroit, Michigan, drew more attention to the scenarios of icing under conditions that have not sufficiently been investigated ${ }^{9}$. The experimental study by Olsen and Walker, carried out by the help of fast picture cameras, revealed that the water transport portion of the model suggested by Messinger was not correct other than during the initial moments of the ice accretion ${ }^{10}$. Consequently, studies to validate and further develop the current icing model were initiated.

Currently, more research on different modes of water transport and heat transfer over the airframe is under way ${ }^{11}$. Some of the investigations include study of water splash, droplet break-up and distortion prior to impact, boundary layer development due to ice roughness, and turbulence effects. Different researchers try to come up with their own alternative mechanisms that are coupled with LEWICE or another aircraft icing software. These efforts are aimed for better ice accretion prediction in a broader range of icing conditions.

The difficulties associated with icing research have multiple components. If the study is going to be experimental, some of the difficulties are determination of scaling factors ${ }^{12}$, artificial turbulence level in the wind tunnel, and generation of the same spectrum of droplet radii in the atmosphere. Another difficulty of the icing research is that experimentally obtained ice shapes are sometimes unrepeatable $\mathrm{e}^{13}$. This fact points to two potential problems: the entire parameter space in icing physics is not known, which prevents a true parametric study, and the viscous effects play a very significant role in icing, which introduces high level of indeterminism into the predictions in case of turbulence. A combination of both problems is also possible.

As far as the numerical studies, due to their deterministic character, they generate ice shapes that are repeatable with the given aircraft geometry and the ambient conditions. However, their capabilities are restricted by the deficiencies in the current understanding of the icing process and the insufficient computational speed of the computers ${ }^{11}$. If a turbulence model is used in order to compensate for the computational limitations, then the actual flight Reynolds number can be matched, but the real details of 
the flow will be lost. On the other hand, if a Direct Numerical Simulation (DNS) approach is going to be used in order to capture the flow details, then the actual flight Reynolds number cannot be achieved due to extremely dense grid and computation time requirements. Nevertheless, some of the numerical research is done by employing the existing turbulence models for flow field and heat transfer calculations, and some others use a potential flow formulation in order to keep time requirements of the unsteady ice accretion process in the affordable and practical range.

The research conducted in the present study is a numerical one that focuses on potential effects of relative humidity and the induced vortices on downstream ice growth. The flow solver is a DNS-type solver with 2D constraint. The flow solver works in terms of stream function and vorticity, which eliminates the need for solving for the pressure during the iterations for the flow. The development and the validation of the flow solver were done as part of the previous work by Huebsch ${ }^{14}$. As a requirement of the first main part of this research on the relative humidity effects, a pressure solver was developed along with the necessary governing equations and boundary conditions. This required a conformal mapping and a shearing transformation applied on the pressure Poisson equation to obtain a final form. Numerical implementation of the pressure solver led to refinements in order to eliminate the numerical instabilities and to ensure accuracy.

The result from the pressure solver is used to obtain the relative humidity variation over the flow field. The relations used to calculate the variation of the relative humidity levels in the flow field are the same ones that are used in atmospheric physics. The key findings from this study show that the vortices shed from a roughness element contain low-pressure cores, which are supersaturated with respect to liquid water and ice. The suction surfaces are also susceptible to the supersaturation due to the low local pressure, which can lead to frost/ice growth and a more complicated flow field and heat transfer characteristics. These findings agree with the idea that the ice roughness itself plays a role in its own development.

The next step in the present relative humidity research was to determine the time scales involved in the relative humidity triggered processes. This was performed based on a numerical experiment that was named as the "box experiment", in which the droplets with the specified properties were exposed to supersaturation or subsaturation effects. The subsequent changes in the droplet radius were traced as a function of time. As a result, it was found that the initial droplet radius severely affects the time required to re-establish the saturation conditions. The smaller droplets needed much less time compared to the large ones, which means small droplets are more vulnerable to the relative humidity affects due to their higher surface area-to-volume ratio. For the particular case considered in this study, the time required for 
the relative humidity effects was found to be 12 milliseconds for a 1micron-diameter droplet, whereas it was as high as 5 seconds for a 20 micron-diameter droplet. It was seen from this numerical experiment that the initial level of the supersaturation does not affect the required time to return to the saturation conditions, but it does affect the final amount of liquid water content of the droplets $(L W C)$. For the same particular case mentioned above, depending on the change in pressure and the flight speed, there can be a $30 \%$ drop, or an increase of $100 \%$ in the $L W C$. Although for the small droplets, the time scale is small enough to see the relative humidity effects in the stagnation region, which mostly overlaps with the direct impingement region, the high amount of liquid water carried by the large droplets surpasses the effect of relative humidity; so, the relative humidity effects are negligible within the direct impingement region. However, the regions beyond the direct impingement are exposed to supersaturation for extended periods. For the region between 10 and $30 \%$ chord location, an extra roughness growth up to $0.4 \mathrm{~mm}$ was calculated over 30 minutes in a particular case.

The second main topic of the present study is the effects of the vortices induced by the existing ice roughness as a contributing mechanism to water transport. A Lagrangian 2-dimensional droplet tracing was assumed to determine the paths of the droplets as they are exposed to gravity, buoyancy and drag forces. The tracing was performed in an unsteady flow field where an unsteady vortex shedding was present. Droplets that would normally not hit the wing or ice surface were affected by the shed vortices and as a result impinged on the surface. This kind of water collection on the airframe can not be captured by the current icing prediction codes because they are using a potential flow solver for the flow field, which can not resolve the vortices present in the field. This result also agrees with the idea that the existing ice roughness plays a role in its own growth downstream of the impingement region.

It was shown that the ratio of the inertia of the droplets and the momentum in the outer regions of a vortex determines how much the droplet is going to be affected by the vortex. Droplets with relatively low inertia tend to follow the streamlines, whereas larger ones interact with the vortex and some of them impinge on the wing surface. For a particular case, the percentage of the extra impingements in the rear of the main ice shape was $6.5 \%$ of the number of droplets impinging on the frontal area of the main ice shape. It was calculated that over a 7 minute period, this amount of impingement has a potential to cause an extra water layer up to $0.24 \mathrm{~mm}$, which is a significant level in terms of its impact on the performance characteristics.

A minor point that was done in the present research was to investigate the frost growth over the wing surface. The existing frost growth models were utilized to make a prediction of the frost growth in case of supersaturation over the wing surface. The results showed that the heat transfer between the frost and the 
airframe results in the frost staying at the specified temperature of the wing surface. Hence if the wing surface is assumed to be at $0{ }^{\circ} \mathrm{C}$, then the frost also grows with a uniform temperature in itself that is equal to the wing surface temperature. The reason for this is that the rate of heat transfer due to convection and latent heat release due the freezing/sublimation is surpassed by the rate of heat transfer due to conduction of heat from the frost base in touch with the wing surface.

The content of this Ph.D. study is prepared as a summation of papers that have been presented at several conferences. Therefore, the subject matter is covered in the order that these studies were presented at the conferences. Below is a brief list of the chapters with the corresponding subject.

1- Chapter II: Pressure field around ice accreted airfoil leading-edge.

2- Chapter III: Potential of local relative humidity to affect aircraft icing.

3- Chapter IV: Mechanisms for downstream ice growth.

4- Chapter V: Further discussion on frost growth and droplet path change.

5- Chapter VI: Conclusions

6- Chapter VII: Recommendations 


\section{CHAPTER II}

\section{PRESSURE FIELD AROUND ICE ACCRETED AIRFOIL LEADING-EDGE}

This study has been presented at the

AIAA $42^{\text {nd }}$ Aerospace Sciences Meeting \& Exhibit

AIAA paper 2004-0059

\section{INTRODUCTION}

Computational studies on aircraft icing have many challenges including generation of a fine and orthogonal grid, highly unsteady and turbulent flow, changing surface geometry, complex ice physics leading to the ice accretion, and the resulting impact on airfoil performance. The extremely active boundary layer and the level of turbulence bring in spatial and temporal fluctuations to the on-going accretion process. Some examples of these fluctuations are vortex shedding, pressure fluctuations, variation of local heat transfer properties, change in water deposition mechanisms to the surface and the altering of the droplet paths. Therefore, a quality computational study must account for these local issues, which require a quality grid at all locations. The computational studies must also have robust theoretical background to retain accuracy over the iterations of an unsteady case.

$\mathrm{LEWICE}^{7}$ is one of the most widely used software programs in icing research. It is a code that uses a potential flow solver coupled with a boundary layer solver. Although the software produces satisfactory results for many cases, it is lacking the details of the flow due to the importance of strong viscous effects. Therefore, the pressure field obtained from this code will have the inviscid flow characteristics and will not reflect the pressure fluctuations due to vortex shedding or flow separation at different scales.

NSU2D is another code that is used in Navier-Stokes solutions over iced airfoils. It is a RANS solver which requires a user defined transition location for the turbulent calculations. Studies by Lee et al. ${ }^{15}$ and Dunn et al. ${ }^{16}$ are examples in which NSU2D has been used. By looking at the pressure distribution over the surface in these studies, it is seen that the code has very good performance over clean airfoils, and has very good agreement with the experiments. However, it has difficulty in capturing the experimental pressure fluctuations and the pressure recovery in the wake of surface roughness features.

Chung and Eddy ${ }^{17}$ used the WIND code for a numerical study over a NLF-0414 airfoil with experimental ice roughness. This code has a RANS solver and utilizes a two-block system which concentrates most of the grid points in the inner region to account for the severe surface roughness. The pressure calculations involve a pre-selected pressure condition: zero normal pressure gradient or normal 
pressure gradient calculation in Cartesian coordinates. Similar to the NSU2D, this code also has problems in following the pressure fluctuations and the pressure recovery downstream of the ice shape.

Another study ${ }^{18}$ in which the WIND code was used shows a similar kind of behavior in the wake of the simulated roughness element. The disagreement between the experiment and the computations grows as the roughness element approaches the leading edge. This is due to the more severe pressure gradient in the leading-edge region and higher roughness height-to-local boundary layer height ratio. This fact and the lack of accuracy in the wake pressure variation point at a pressure gradient related problem for the computations. Possible reasons of this problem are the restrictions of the RANS model, or validity of the pressure boundary condition, or orthogonality problems of the grid.

The present study is a continuation of an in-house code development study for incompressible Navier-Stokes. This code focuses on the leading-edge of the airfoil where most of the roughness related phenomena occur. The solver is a DNS type solver with 2-D restriction. The full incompressible N-S equations are solved in the stream function-vorticity formulation. Although the $2 \mathrm{D}$ character limits the capability of the code, the grid generation capabilities, which stem from robust analytic techniques, provide orthogonal or near-orthogonal grid and enhanced accuracy for the computations. The theoretical background of the code involves conformal mappings and the Fourier transform to handle surface roughness. The details of the formulation and other information about the code can be found in references [14], [19], and [20].

Initial work on the pressure solver was made by Bhaskaran ${ }^{20}$ by transforming the pressure Poisson equation through a conformal mapping into the computational plane. In that study, the primary focus was to understand the unsteady phenomena around a clean airfoil leading-edge, where the airfoil leading-edge was simulated by the leading-edge of a parabola. The study included stream function-vorticity solutions along with the pressure field calculations over a clean parabola in a pitch-up maneuver at a constant rate.

In the present study, the primary focus is to understand the pressure field around an airfoil leadingedge with complex ice roughness (example: Figure 1). Therefore, the ice roughness geometry and the actual airfoil geometry need to be incorporated into the governing equations. The corresponding boundary condition for the pressure solver also needs to be modified. The derivations for the pressure Poisson equation along with the corresponding boundary condition are outlined in the present study. The validation of the new pressure solver is presented both qualitatively and quantitatively. 


\section{GOVERNING EQUATIONS}

The governing flow equations are the stream function-vorticity version of the 2-D incompressible, unsteady Navier-Stokes equations, which are shown below in non-dimensional form. The information related to the flow solver can be found in the relevant references ([14], [19], and [20]).

$$
\begin{gathered}
\omega_{t}+\psi_{y} \omega_{x}-\psi_{x} \omega_{y}=\operatorname{Re}^{-1}\left(\omega_{x x}+\omega_{y y}\right) \\
\psi_{x x}+\psi_{y y}=-\omega
\end{gathered}
$$

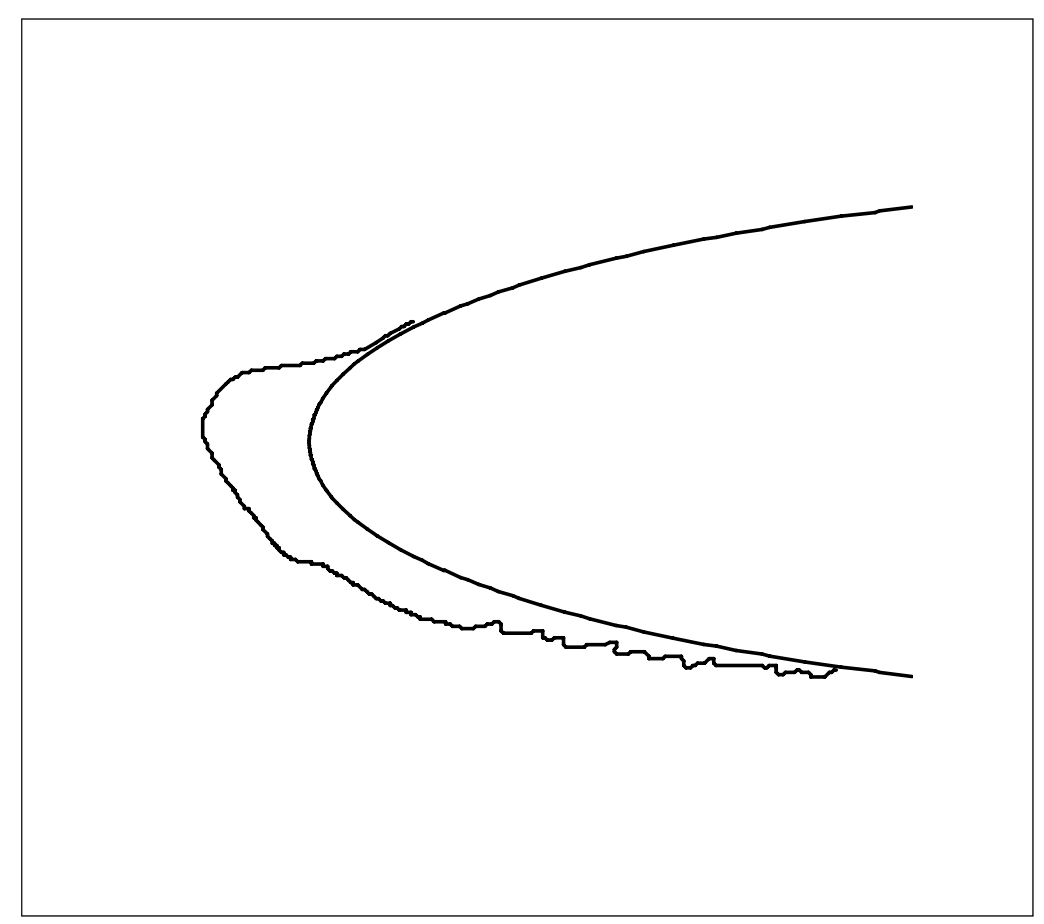

Figure 1. NACA 0012 leading-edge and the ice shape (I-1998-000092).

\section{Calculation of Pressure}

Since the governing flow equations can be solved independent of pressure, a post processing step is necessary to solve for the pressure field using the converged flow $(\psi-\omega)$ solution. For the solution of the pressure field, the non-dimensional pressure coefficient is used, which is defined as follows with the help of the static pressure and the dynamic pressure at infinity.

$$
C_{p}=\frac{\left(P-P_{\infty}\right)}{\frac{1}{2} \rho V_{\infty}^{2}}
$$


The Poisson equation that is used to solve for this non-dimensionalized pressure is given below in its closed form.

$$
\nabla^{2} C_{p}=-2 \nabla \cdot[(\vec{V} \cdot \nabla) \vec{V}]
$$

Several transformations are applied to the Poisson equation to move from physical space to computational space, which correspond to the transformations for the flow equations. More information about these transformations can be found in References [14], [19], and [20]. Here, a brief explanation is provided in steps, as applied to the pressure Poisson equation:

1. The airfoil and the corresponding surface roughness (e.g., ice roughness) are first nondimensionalized by the leading-edge radius of the airfoil $\left(x-y \rightarrow x^{\prime}-y^{\prime}\right)$. This type of nondimensionalization introduces the ice roughness and the airfoil geometry as perturbations to the parabola surface (see Figure 2-a). This modification to the geometry has been adopted to embed the geometry of interest into the flow solver that works on a parabola as its base geometry. It also allows the focusing of the available computational power and grid resolution around the airfoil where very fine local flow details are of interest. Another advantage of using a parabola as the base geometry is that the apparent Reynolds number is reduced due to the fact that the characteristic length for the flow reduces to the leading-edge radius (ler). This Reynolds number is correlated to the typical chord value through the following expression:

$$
R e_{l e r}=\operatorname{Re}(l e r / c)
$$

2. Transformation from the non-dimensional physical plane $\left(x^{\prime}-y^{\prime}\right)$ to the transformed $\left(\xi^{\prime}-\eta^{\prime}\right)$ plane using the below formulae.

$$
x^{\prime}=\frac{\xi^{\prime 2}-\eta^{\prime 2}}{2}, y^{\prime}=\xi^{\prime} \eta^{\prime}
$$

The effect of this transformation on the surface geometry is shown pictorially in Figure 2-b. The base surface, which is a parabola in the $x^{\prime}-y^{\prime}$ plane, becomes a straight line in the new $\xi^{\prime}-\eta^{\prime}$ plane through conformal mapping. The corresponding perturbations (airfoil and/or ice roughness) present on that parabola become perturbations to this straight line (Figure 3). After this conformal mapping, the pressure Poisson equation takes the form shown below:

$$
C_{p_{\xi^{\prime \xi^{\prime}}}}+C_{p_{\eta^{\prime} \eta^{\prime}}}=4\left[\left(u_{\xi^{\prime}}+\frac{v \eta^{\prime}}{\xi^{\prime 2}+\eta^{\prime 2}}\right)\left(v_{\eta^{\prime}}+\frac{u \xi^{\prime}}{\xi^{\prime 2}+\eta^{\prime 2}}\right)-\left(v_{\xi^{\prime}}-\frac{u \eta^{\prime}}{\xi^{\prime 2}+\eta^{\prime 2}}\right)\left(u_{\eta^{\prime}}-\frac{v \xi^{\prime}}{\xi^{\prime 2}+\eta^{\prime 2}}\right)\right]
$$

Velocities in the $\xi^{\prime}$ and $\eta^{\prime}$ directions ( $u$ and $v$, respectively) and their derivatives are obtained from the converged stream function field as follows: 


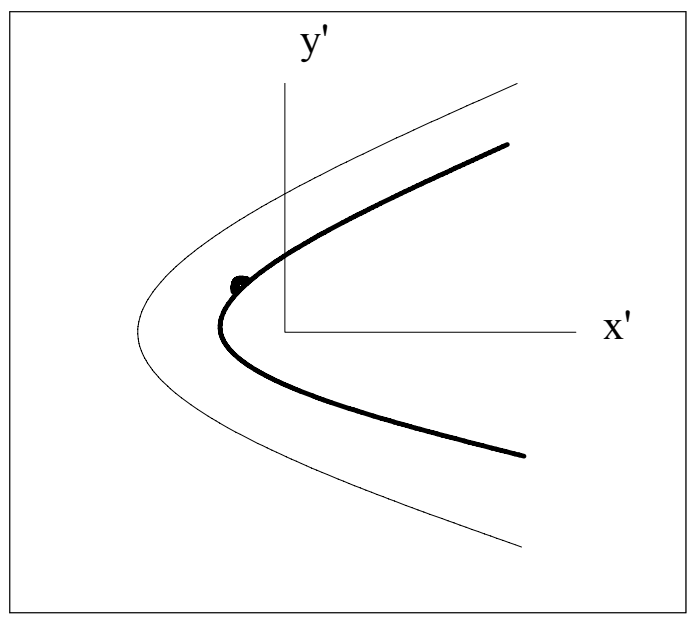

a) $x^{\prime}-y^{\prime}$ plane

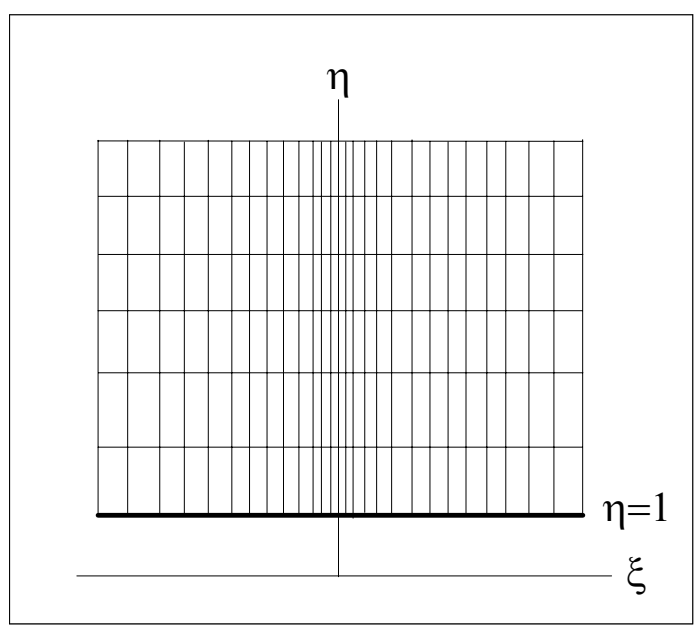

c) $\xi-\eta$

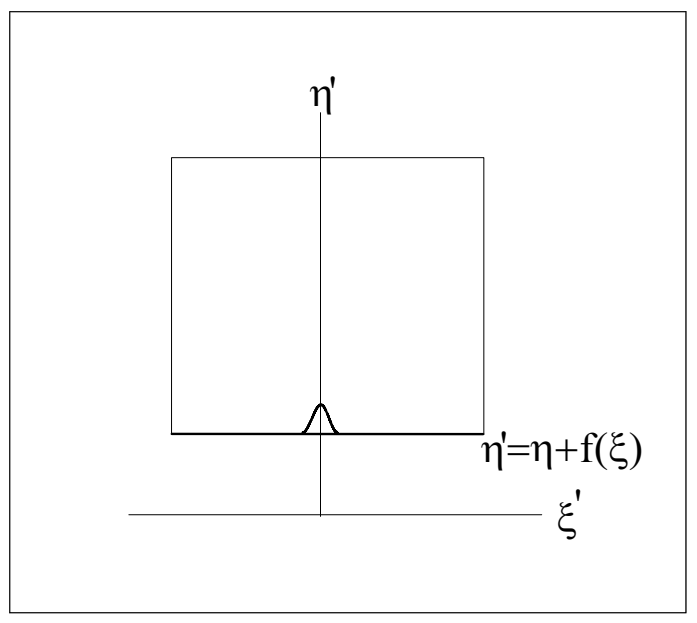

b) $\xi^{\prime}-\eta^{\prime}$ plane

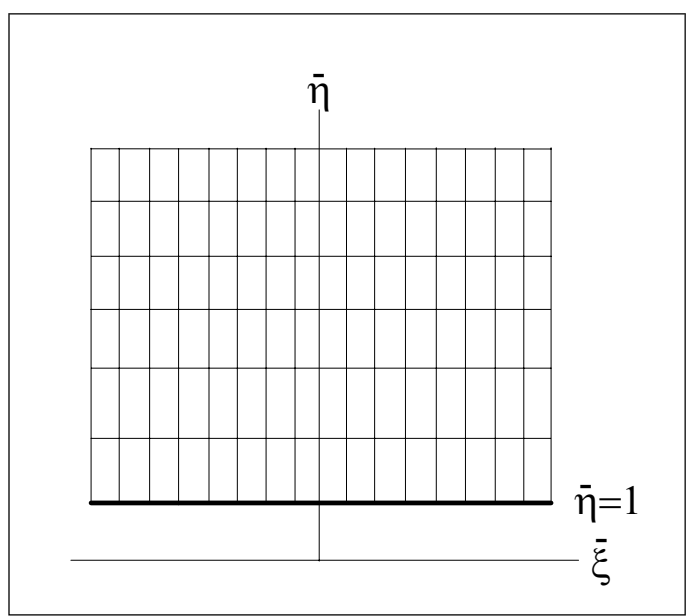

d) $\bar{\xi}-\bar{\eta}$

Figure 2. Geometrical depiction of the transformations.

$$
\begin{aligned}
& \vec{V}=\nabla \psi \times \hat{k} \quad u=\frac{\psi_{\eta^{\prime}}}{\sqrt{\xi^{\prime 2}+\eta^{\prime 2}}} \quad v=-\frac{\psi_{\xi^{\prime}}}{\sqrt{\xi^{\prime 2}+\eta^{\prime 2}}} \\
& u_{\xi^{\prime}}=\frac{\psi_{\xi^{\prime} \eta^{\prime}}}{\sqrt{\xi^{\prime 2}+\eta^{\prime 2}}}-\frac{u \xi^{\prime}}{\xi^{\prime 2}+\eta^{\prime 2}} \quad u_{\eta^{\prime}}=\frac{\psi_{\eta^{\prime} \eta^{\prime}}}{\sqrt{\xi^{\prime 2}+\eta^{\prime 2}}}-\frac{u \eta^{\prime}}{\xi^{\prime 2}+\eta^{\prime 2}} \\
& v_{\xi^{\prime}}=-\frac{\psi_{\xi^{\prime} \xi^{\prime}}}{\sqrt{\xi^{\prime 2}+\eta^{\prime 2}}}-\frac{v \xi^{\prime}}{\xi^{\prime 2}+\eta^{\prime 2}} \quad v_{\eta^{\prime}}=-\frac{\psi_{\xi^{\prime} \eta^{\prime}}}{\sqrt{\xi^{\prime 2}+\eta^{\prime 2}}}-\frac{v \eta^{\prime}}{\xi^{\prime 2}+\eta^{\prime 2}}
\end{aligned}
$$




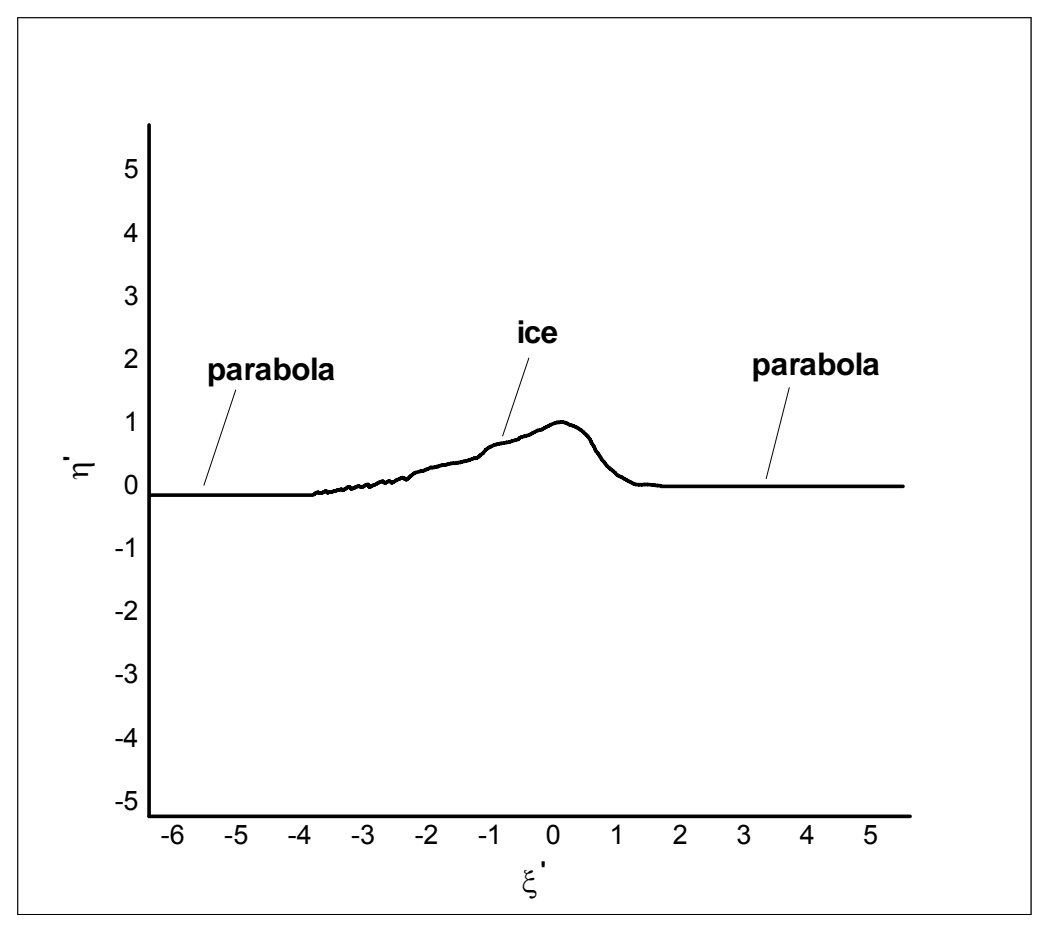

Figure 3. Parabola and the ice shape (I-1998-000092) in the transformed plane.

3. Separating the perturbation geometry from the base surface is achieved by making use of the Prandtl transposition:

$$
\xi^{\prime}=\xi, \quad \eta^{\prime}=\eta+f(\xi)
$$

where $f(\xi)$ is a single-valued expression representing the shape of the perturbation geometry. The Prandtl transposition transforms the geometry to the $\xi-\eta$ plane (Figure 2-c), and as a result the surface perturbation geometry and its derivatives are introduced to the governing equations as $f, f_{\tilde{\xi}}, f_{\xi \xi}$. These three terms have to be expressed analytically so that no artificial effects are introduced due to a jump discontinuity in the geometry or its derivatives. In the present study, the $f$ related terms express the ice roughness shape or other roughness elements. As seen in Figure 3, the roughness shape resembles a complex signal, which can be expressed by sine and cosine functions through the Fourier analysis ${ }^{19}$. Further details of this process can also be found in the master's thesis "Ice Shape Modeling Enhancement For 2-D Incompressible Local-Flow Navier-Stokes" by Egemen Ogretim.

After separating the perturbation (roughness) geometries using the Prandtl transposition, the pressure Poisson equation and the related velocity terms take the following form.

$$
C_{p_{\xi \xi}}+\left(1+f_{\xi}^{2}\right) C_{p_{\eta \eta}}-f_{\xi \xi} C_{p_{\eta}}-2 f_{\xi} C_{p_{\xi \eta}}=R
$$

where 


$$
\begin{gathered}
R=4\left[\left(u_{\xi}-f_{\xi} u_{\eta}+\frac{v(\eta+f)}{\xi^{2}+(\eta+f)^{2}}\right)\left(v_{\eta}+\frac{u \xi}{\xi^{2}+(\eta+f)^{2}}\right)-\left(v_{\xi}-f_{\xi} v_{\eta}-\frac{u \eta}{\xi^{2}+(\eta+f)^{2}}\right)\left(u_{\eta}-\frac{v \xi}{\xi^{2}+(\eta+f)^{2}}\right)\right] \\
u=\frac{\psi_{\eta}}{\sqrt{\xi^{2}+(\eta+f)^{2}}} \quad v=-\frac{\psi_{\xi}-f_{\xi} \psi_{\eta}}{\sqrt{\xi^{2}+(\eta+f)^{2}}}=-\frac{\psi_{\xi}}{\sqrt{\xi^{2}+(\eta+f)^{2}}}+f_{\xi} u \\
u_{\xi}=\frac{\psi_{\xi \eta}}{\sqrt{\xi^{2}+(\eta+f)^{2}}}-\frac{u\left(\xi+f_{\xi}(\eta+f)\right)}{\xi^{2}+(\eta+f)^{2}} \quad v_{\xi}=-\frac{\left(\psi_{\xi \xi}-f_{\xi \xi} \psi_{\eta}-f_{\xi} \psi_{\xi \eta}\right)}{\sqrt{\xi^{2}+(\eta+f)^{2}}}-\frac{v\left(\xi+f_{\xi}(\eta+f)\right)}{\xi^{2}+(\eta+f)^{2}} \\
u_{\eta}=\frac{\psi_{\eta \eta}}{\sqrt{\xi^{2}+(\eta+f)^{2}}}-\frac{u(\eta+f)}{\xi^{2}+(\eta+f)^{2}} \quad v_{\eta}=-\frac{\psi_{\xi \eta}-f_{\xi} \psi_{\eta \eta}}{\sqrt{\xi^{2}+(\eta+f)^{2}}}-\frac{v(\eta+f)}{\xi^{2}+(\eta+f)^{2}}
\end{gathered}
$$

4. A clustering and stretching of grid points which transform the governing equations to $\bar{\xi}, \bar{\eta}$ plane (Figure 2-d).

\section{Initial Condition}

The pressure field is initialized by the use of the inviscid stream function describing a stagnation flow field. The reason for using this stagnation flow is that the flow over a parabola surface becomes a stagnation flow over a flat plate after the conformal mapping. As the perturbation geometries are introduced, this inviscid stream function becomes:

$$
\psi_{i n v}=(\xi+K)(\eta+f-1)
$$

where $\mathrm{K}$ is a parameter that represents the angle of attack. Using this equation, the velocity components, and the inviscid pressure field can be obtained as follows:

$$
\begin{gathered}
u_{i n v}=\frac{\xi+K}{\sqrt{\xi^{2}+(\eta+f)^{2}}} \quad v_{i n v}=\frac{-\left[(\eta+f-1)+f_{\xi}(\xi+K)\right]}{\sqrt{\xi^{2}+(\eta+f)^{2}}} \\
C_{p, i n v}=1-\left(u_{i n v}^{2}+v_{i n v}^{2}\right)=-\frac{K^{2}+2 K \xi-2(\eta+f)+1}{\xi^{2}+(\eta+f)^{2}}
\end{gathered}
$$

\section{Far Upstream Boundary Condition}

Since the far upstream is not going to be affected by the presence of the viscous flow around the surface, the inviscid pressure values are preserved as the far upstream boundary condition. 


\section{Far Downstream Boundary Condition}

According to Davis et al. ${ }^{21}$, in case of a stagnation flow over a flat plate, the pressure at the far downstream approaches the inviscid values. Therefore, the inviscid pressure values are retained as the far downstream boundary condition.

\section{Wall Boundary Condition}

For the clean parabola, the normal direction in the transformed plane is the $\eta^{\prime}$ direction. The wall boundary condition for pressure in this case was derived and utilized in the dissertation study by Bhaskaran $^{20}$ as given below.

$$
C_{p_{\eta}}=2 \operatorname{Re}^{-1} \omega_{\xi^{\prime}}
$$

The main focus of the present study is the derivation, implementation, and validation of the wall boundary condition in case of a perturbation on the parabola surface. In order to save space, some of the details are left to reference [20].

The derivation starts with the primitive variable version of the Navier-Stokes equations, and then noslip boundary condition is applied.

$$
\vec{V}_{t}+(\vec{V} . \nabla) \vec{V}=-\frac{1}{2} \nabla C_{p}+R e^{-1} \nabla^{2} \vec{V} \text {, but } \vec{V}=0 \text { at the wall, so, } \nabla C_{p}=2 R e^{-1} \nabla^{2} \vec{V} \text {, where } \vec{V}=u \hat{e}_{\xi^{\prime}}+v \hat{e}_{\eta^{\prime}}
$$

The definitions for gradient and Laplacian in the transformed $\left(\xi^{\prime}-\eta^{\prime}\right)$ plane are:

$$
\nabla \phi=\frac{1}{h}\left(\phi_{\xi^{\prime}} \hat{e}_{\xi^{\prime}}+\phi_{\eta^{\prime}} \hat{e}_{\eta^{\prime}}\right) \quad \nabla^{2} \vec{A}=\frac{1}{h^{2}}\left(\frac{\partial}{\partial \xi^{\prime 2}}+\frac{\partial}{\partial \eta^{\prime 2}}\right)\left(A_{1} \hat{e}_{\xi^{\prime}}+A_{2} \hat{e}_{\eta^{\prime}}\right)
$$

where $h=\sqrt{\xi^{\prime 2}+\eta^{\prime 2}}$. The Laplacian expression needs special attention when expanding. This is due to the fact that the unit vectors in the $\xi^{\prime}-\eta^{\prime}$ plane change as a function of the local coordinates. Taking this into account, the following is obtained:

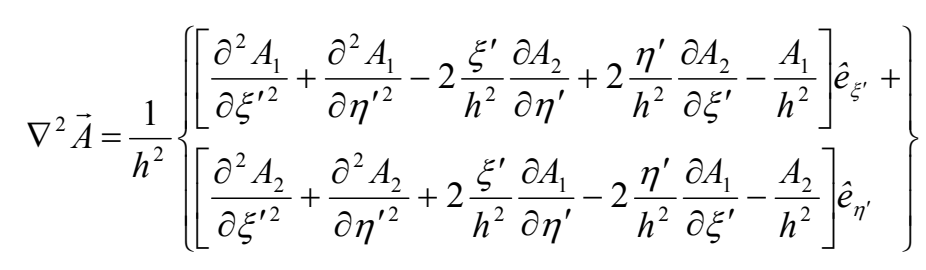

Then, the pressure equation at the wall boundary becomes, 


$$
\frac{1}{h}\left(\frac{\partial C_{p}}{\partial \xi^{\prime}} \hat{e}_{\xi^{\prime}}+\frac{\partial C_{p}}{\partial \eta^{\prime}} \hat{e}_{\eta^{\prime}}\right)=R e^{-1} \frac{2}{h^{2}}\left\{\begin{array}{l}
{\left[\frac{\partial^{2} u}{\partial \xi^{\prime 2}}+\frac{\partial^{2} u}{\partial \eta^{\prime 2}}-2 \frac{\xi^{\prime}}{h^{2}} \frac{\partial v}{\partial \eta^{\prime}}+2 \frac{\eta^{\prime}}{h^{2}} \frac{\partial v}{\partial \xi^{\prime}}-\frac{u}{h^{2}}\right] \hat{e}_{\xi^{\prime}}+} \\
{\left[\frac{\partial^{2} v}{\partial \xi^{\prime 2}}+\frac{\partial^{2} v}{\partial \eta^{\prime 2}}+2 \frac{\xi^{\prime}}{h^{2}} \frac{\partial u}{\partial \eta^{\prime}}-2 \frac{\eta^{\prime}}{h^{2}} \frac{\partial u}{\partial \xi^{\prime}}-\frac{v}{h^{2}}\right] \hat{e}_{\eta^{\prime}}}
\end{array}\right\}
$$

Using the no slip condition, and taking the dot product of both sides of the Equation 18 with $\hat{e}_{\xi^{\prime}}$ and $\hat{e}_{\eta^{\prime}}$ separately, one gets two pressure gradient equations: one in the $\xi^{\prime}$ direction and one in the $\eta^{\prime}$ direction:

$$
\begin{aligned}
& \frac{\partial C_{p}}{\partial \xi^{\prime}}=\frac{2 R e^{-1}}{h}\left[\frac{\partial^{2} u}{\partial \xi^{\prime 2}}+\frac{\partial^{2} u}{\partial \eta^{\prime 2}}-2 \frac{\xi^{\prime}}{h^{2}} \frac{\partial v}{\partial \eta^{\prime}}+2 \frac{\eta^{\prime}}{h^{2}} \frac{\partial v}{\partial \xi^{\prime}}\right] \\
& \frac{\partial C_{p}}{\partial \eta^{\prime}}=\frac{2 R e^{-1}}{h}\left[\frac{\partial^{2} v}{\partial \xi^{\prime 2}}+\frac{\partial^{2} v}{\partial \eta^{\prime 2}}+2 \frac{\xi^{\prime}}{h^{2}} \frac{\partial u}{\partial \eta^{\prime}}-2 \frac{\eta^{\prime}}{h^{2}} \frac{\partial u}{\partial \xi^{\prime}}\right]
\end{aligned}
$$

After this point, the Prandtl transposition (shearing transformation) is applied in order to suppress the perturbations on the parabola surface. As a result of this, the perturbation geometry is embedded in the wall boundary condition equations. The chain rules that are used for the application of the Prandtl transposition are:

$$
\begin{array}{lc}
\frac{\partial}{\partial \xi^{\prime}}=\frac{\partial}{\partial \xi}-f_{\xi} \frac{\partial}{\partial \eta} & \frac{\partial}{\partial \eta^{\prime}}=\frac{\partial}{\partial \eta} \\
\frac{\partial^{2}}{\partial \xi^{\prime 2}}=\frac{\partial^{2}}{\partial \xi^{2}}-f_{\xi \xi} \frac{\partial}{\partial \eta}-2 f_{\xi} \frac{\partial^{2}}{\partial \xi \partial \eta}+f_{\xi}^{2} \frac{\partial^{2}}{\partial \eta^{2}} & \frac{\partial^{2}}{\partial \eta^{\prime 2}}=\frac{\partial^{2}}{\partial \eta^{2}}
\end{array}
$$

Making the relevant changes due to the Prandtl transposition, the gradient equations become:

$$
\begin{aligned}
& \frac{\partial C_{p}}{\partial \xi}-f_{\xi} \frac{\partial C_{p}}{\partial \eta}=\frac{2 R e^{-1}}{h}\left[\frac{\partial^{2} u}{\partial \xi^{2}}-f_{\xi \xi} \frac{\partial u}{\partial \eta}-2 f_{\xi} \frac{\partial^{2} u}{\partial \xi \partial \eta}+\left(1+f_{\xi}^{2}\right) \frac{\partial^{2} u}{\partial \eta^{2}}-\frac{2 \xi}{h^{2}} \frac{\partial v}{\partial \eta}+\frac{2(\eta+f)}{h^{2}}\left(\frac{\partial v}{\partial \xi}-f_{\xi} \frac{\partial v}{\partial \eta}\right)\right] \\
& \frac{\partial C_{p}}{\partial \eta}=\frac{2 R e^{-1}}{h}\left[\frac{\partial^{2} v}{\partial \xi^{2}}-f_{\xi \xi} \frac{\partial v}{\partial \eta}-2 f_{\xi} \frac{\partial^{2} v}{\partial \xi \partial \eta}+\left(1+f_{\xi}^{2}\right) \frac{\partial^{2} v}{\partial \eta^{2}}+2 \frac{\xi}{h^{2}} \frac{\partial u}{\partial \eta}-2 \frac{(\eta+f)}{h^{2}}\left(\frac{\partial u}{\partial \xi}-f_{\xi} \frac{\partial u}{\partial \eta}\right)\right]
\end{aligned}
$$

But the velocities along the surface are going to be zero due to no slip; and the corresponding derivatives of the velocities along the surface are also zero. So, $\frac{\partial \vec{V}}{\partial \xi}=0$ and $\frac{\partial^{2} \vec{V}}{\partial \xi^{2}}=0$. Then the gradient equations become: 


$$
\begin{gathered}
\frac{\partial C_{p}}{\partial \xi}-f_{\xi} \frac{\partial C_{p}}{\partial \eta}=\frac{2 R e^{-1}}{h}\left[-f_{\xi \xi} \frac{\partial u}{\partial \eta}-2 f_{\xi} \frac{\partial^{2} u}{\partial \xi \partial \eta}+\left(1+f_{\xi}^{2}\right) \frac{\partial^{2} u}{\partial \eta^{2}}-\frac{2 \xi}{h^{2}} \frac{\partial v}{\partial \eta}-\frac{2(\eta+f)}{h^{2}} f_{\xi} \frac{\partial v}{\partial \eta}\right] \\
\frac{\partial C_{p}}{\partial \eta}=\frac{2 R e^{-1}}{h}\left[-f_{\xi \xi} \frac{\partial v}{\partial \eta}-2 f_{\xi} \frac{\partial^{2} v}{\partial \xi \partial \eta}+\left(1+f_{\xi}^{2}\right) \frac{\partial^{2} v}{\partial \eta^{2}}+\frac{2}{h^{2}}\left(\xi+f_{\xi}(\eta+f)\right) \frac{\partial u}{\partial \eta}\right]
\end{gathered}
$$

Further combining the Equations 23 and 24, one gets an equation that accounts for the change of pressure along the solid surface:

$$
\frac{\partial C_{p}}{\partial \xi}=\frac{2 R e^{-1}}{h}\left\{\begin{array}{l}
\frac{\partial u}{\partial \eta}\left[-f_{\xi \xi}+\frac{2 f_{\xi} \xi}{h^{2}}+\frac{2 f_{\xi}^{2}(\eta+f)}{h^{2}}\right]-\frac{\partial v}{\partial \eta}\left[f_{\xi} f_{\xi \xi}+\frac{2 \xi}{h^{2}}+\frac{2 f_{\xi}(\eta+f)}{h^{2}}\right]+ \\
\left(1+f_{\xi}^{2}\right) \frac{\partial^{2} u}{\partial \eta^{2}}+\left(1+f_{\xi}^{2}\right) f_{\xi} \frac{\partial^{2} v}{\partial \eta^{2}}-2 f_{\xi} \frac{\partial^{2} u}{\partial \xi \partial \eta}-2 f_{\xi}^{2} \frac{\partial^{2} v}{\partial \xi \partial \eta}
\end{array}\right\}
$$

It is important to note here that this equation returns to the boundary condition along the wall for the clean parabola case, if the $f$ related terms are set to zero. Equation 25 is a ready to use equation as a boundary condition. However, for the present study, the derivation is further continued to relate the velocity terms to the vorticity/stream function terms. Therefore, using the definition of the stream function and vorticity, and the no slip boundary condition, the following are obtained:

$$
\begin{gathered}
\frac{\partial u}{\partial \eta}=-\frac{h \omega}{\left(1+f_{\xi}^{2}\right)} \\
\frac{\partial^{2} u}{\partial \xi \partial \eta}=\frac{\psi_{\xi \eta \eta}}{h}+\frac{\omega\left\lfloor\xi+f_{\xi}(\eta+f)\right]}{h\left(1+f_{\xi}^{2}\right)}=-\frac{f_{\xi} h \omega}{\left(1+f_{\xi}^{2}\right)} \quad \frac{\partial^{2} v}{\partial \xi \partial \eta}=\frac{f_{\xi} \psi_{\xi \eta \eta}}{h}+\frac{\left.f_{\xi} \omega \mid \xi+f_{\xi}(\eta+f)\right]}{h\left(1+f_{\xi}^{2}\right)}-\frac{f_{\xi \xi} h \omega}{\left(1+f_{\xi}^{2}\right)} \\
\frac{\partial^{2} u}{\partial \eta^{2}}=\frac{2 \omega(\eta+f)}{h\left(1+f_{\xi}^{2}\right)}+\frac{\psi_{\eta \eta \eta}}{h} \\
\psi_{\eta \eta \eta}=\frac{h^{2}}{\left(1+f_{\xi}^{2}\right)}\left[\frac{2 f_{\xi}}{h^{2}} \psi_{\xi \eta \eta}-\omega_{\eta}-\frac{2 \omega(\eta+f)}{h^{2}}-\frac{\left(\psi_{\xi \eta \eta}-f_{\xi \xi} \psi_{\eta \eta \eta}\right)}{h}\right. \\
\psi_{\xi \eta \eta}=\frac{h^{2}}{\left.\left(1+f_{\xi}^{2}\right)\right]}\left[\frac{2 f_{\xi}^{2} f_{\xi \xi} \omega}{\left(1+f_{\xi}^{2}\right)}-\omega_{\xi}-\frac{2 \omega\left(\xi+f_{\xi}(\eta+f)\right)}{h^{2}}\right]
\end{gathered}
$$

The order of use for the above given equations for a surface pressure calculation is as follows:

1. obtain $\omega_{\xi}$ and $\omega_{\eta}$ using the converged flow field 
2. calculate the $\psi_{\xi \eta \eta}$ and $\psi_{\eta \eta \eta}$ terms

3. calculate the velocity derivative terms

4. calculate the $C_{p_{5}}$

\section{Numerical Method}

The pressure solver that is considered in the present study is a post-process step for the flow solver, since the flow solver is based on the stream function and vorticity. Therefore, the pressure solver starts from an already converged flow field. The pressure solver inputs this flow field and uses $2^{\text {nd }}$-order accurate finite differences to solve for the pressure Poisson equation. A Gauss-Siedel iteration scheme is used until a specified convergence level or a number of iterations. The discretization of the wall boundary

equations involve a $2^{\text {nd }}$ order one-sided differencing for the $\omega_{\eta}$ term, and a $2^{\text {nd }}$ order central differencing for the rest of the derivative terms.

In order to avoid the checkerboard instability, a staggered-grid (see Figure 4) approach is used for calculating the velocities and their derivatives. $C_{p}$ is calculated at the cell center, whereas the velocities are calculated at the cell faces. An averaging is used in order to get the value of the velocity at the cell center. The relative convergence rate can be as low as $10^{-4}$ or $10^{-5}$ depending on the complexity of the surface roughness, grid size and the available computation time.

For the wall pressure values, a formula that is along the solid surface is preferred in order to avoid instability due to the feedback between the interior field and the surface. Equation 25 gives the pressure gradient along the surface as function of the geometry and the flow variables, which are already known from the converged flow field. So, the four steps outlined in the wall boundary condition section are used in order to obtain the solid surface pressure values prior to the iterations on the interior field.

Due to the geometrical characteristic of this study, the air stream aft of the airfoil in an actual case cannot be simulated due to the existence of the parabola surface in the model. Therefore, there is a need for aft-airfoil boundary condition to emulate the actual case. This is done by specifying the wall $C_{p}$ value as 0 for these aft-airfoil locations. In order to calculate the surface pressure in the region around the stagnation point, a small pocket region is selected, and the pressure in this region is calculated by Equation 16 with a single iteration. Having specified the stagnation region and the aft-airfoil boundary condition, the airfoil surface pressure values are implicitly solved for by using a second-order central differencing as shown below. 


$$
C_{p_{\xi \xi}}=\frac{\partial}{\partial \xi}\left(\frac{\partial C_{p}}{\partial \xi}\right)=\frac{\left(C_{p_{\xi}}\right)_{i+1 / 2}-\left(C_{p_{\xi}}\right)_{i-1 / 2}}{\Delta \xi}
$$

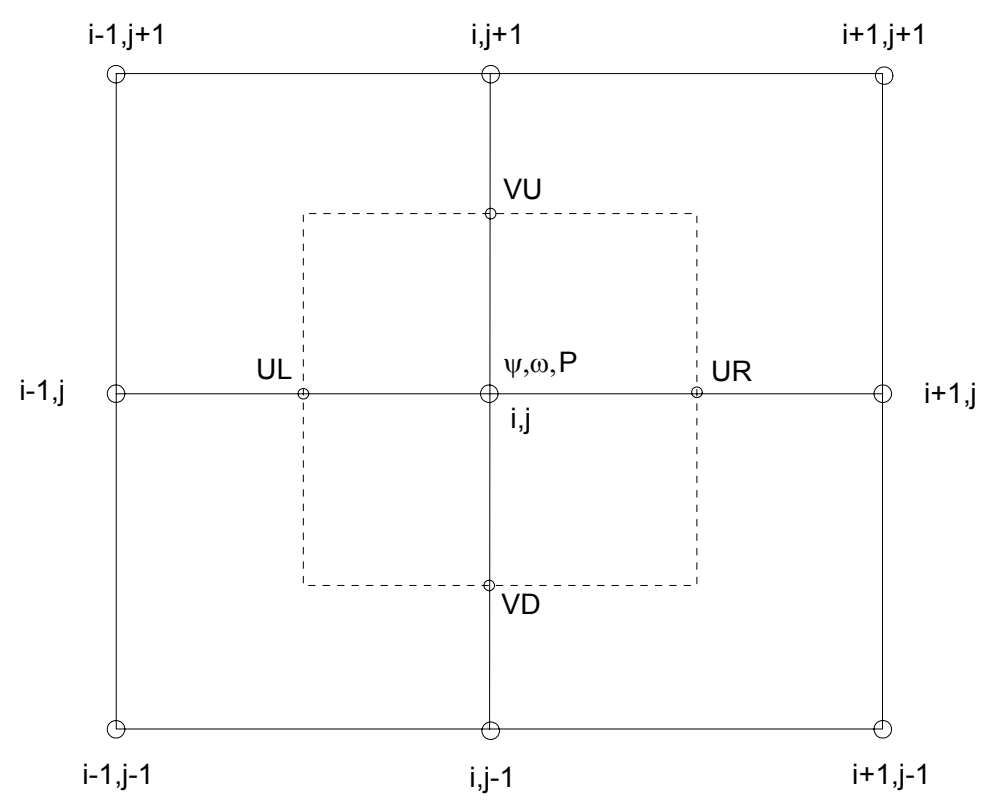

Figure 4. Depiction of the staggered grid approach.

\section{RESULTS}

The results of the present study consist of the validation of the in-house pressure solver and its applications to the flow field around a NACA 0012 airfoil with experimental ice shapes. The grid and the flow solver combination is a DNS-type solver with a 2D constraint. It is capable of capturing the largescale and small-scale flow features that are inherent to unsteady viscous flow past surface roughness. An example of the grid used can be seen in Figure 5 and Figure 6. Figure 5 shows an overall view of the grid around an airfoil with experimental ice shape along with a zoom-in (40 times enlarged) view on the leading-edge region. Figure 6 provides a close-up of this grid $(\approx 3500$ times enlarged $)$ near the ice roughness on the lower portion of the leading-edge. As these figures clearly show, the level of resolution obtained by the $8 \times 10^{5}$ grid points is sufficient to capture the very small details of the flow surrounding the complex roughness shapes, provided that the Reynolds number is within the acceptable range for a DNS. Grid clustering is used to place a dense streamwise uniform grid in the perturbation region and grid stretching is used to cluster grid points near and in the boundary layer. It is important to have a dense grid 
not only within the boundary layer but also in the vicinity of the boundary layer due to the highly active vortex dynamics over the surface and the consequent pressure field.

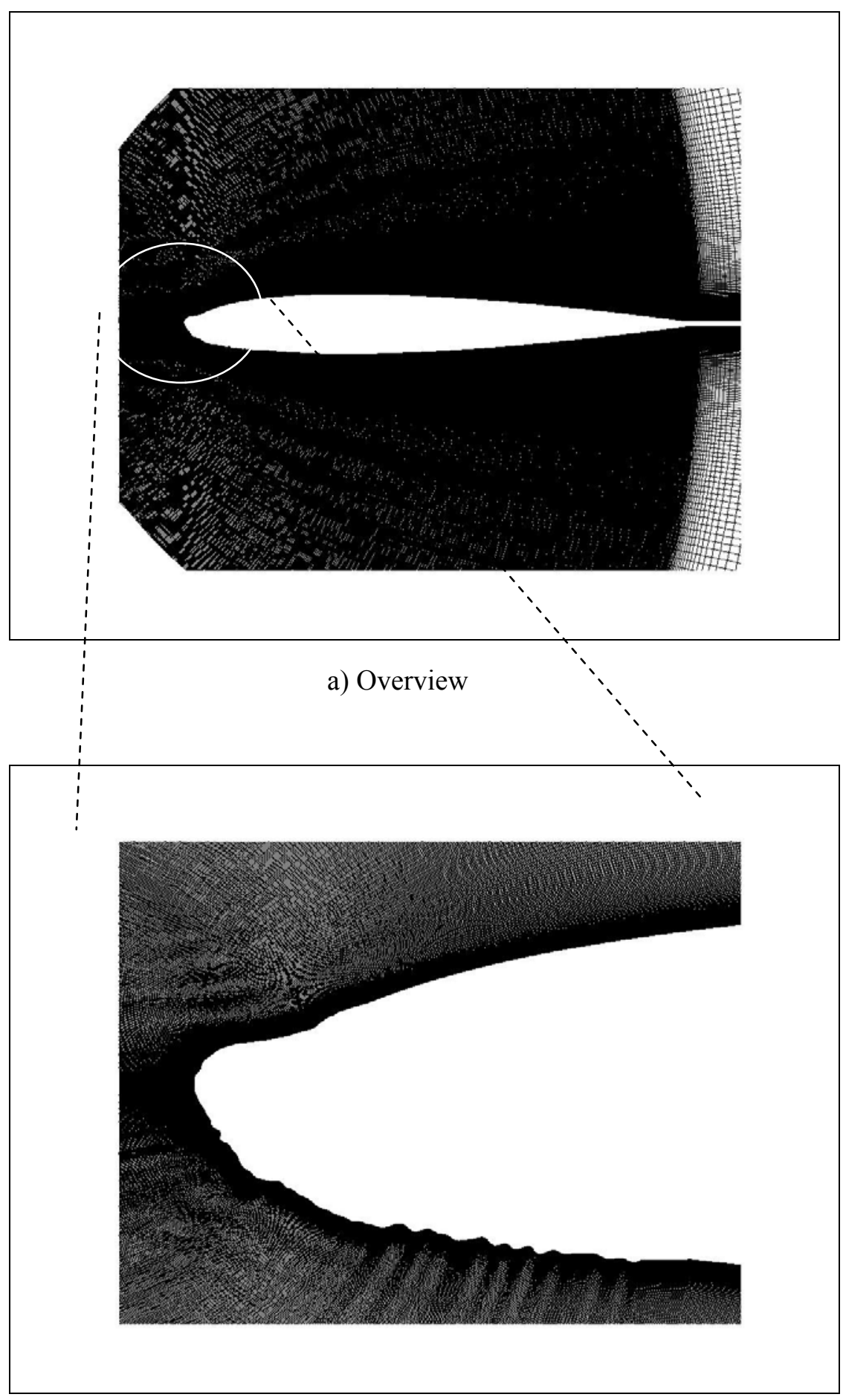

b) $\mathrm{x} 40$ zoom-in

Figure 5. Overview and a zoom in of the mesh over the ice shape (I-1998-000092). 


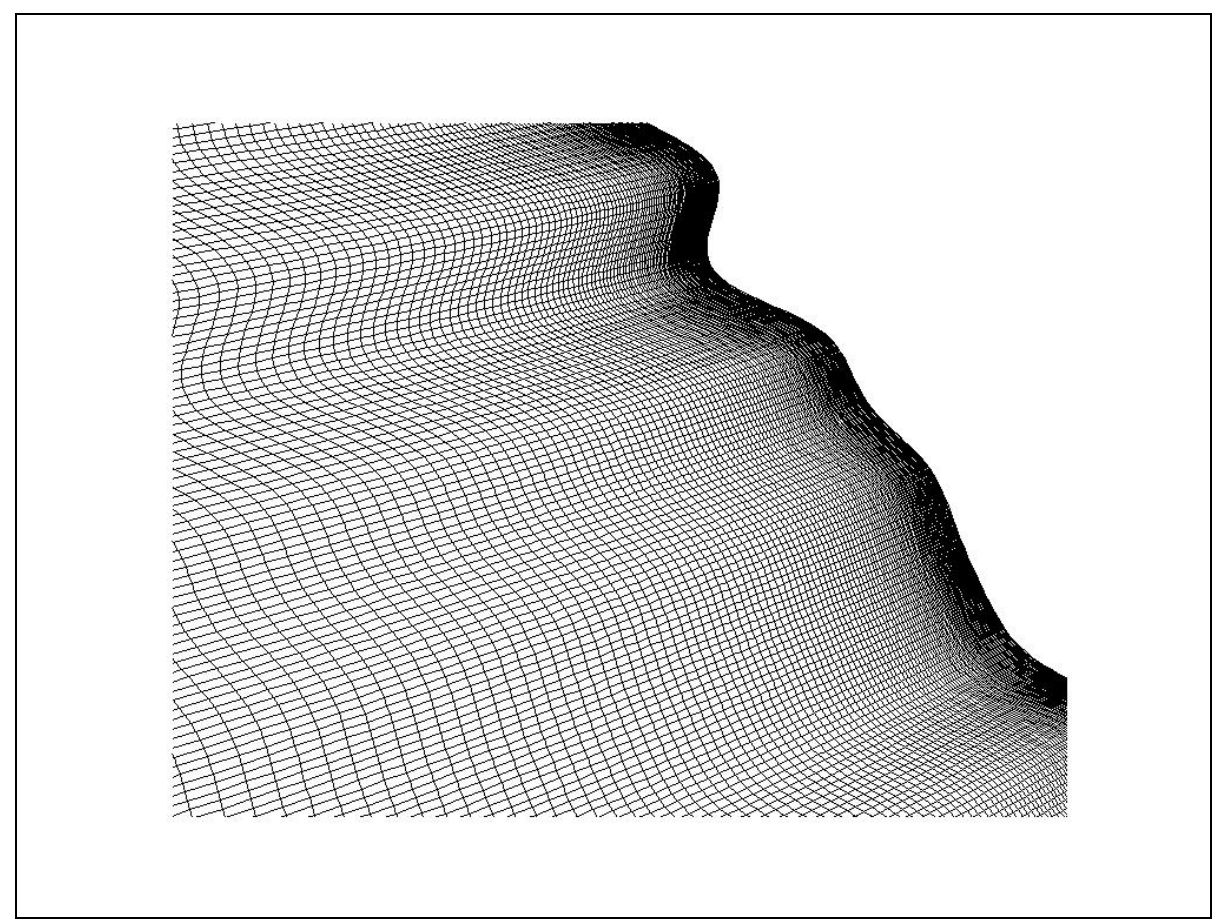

Figure 6. A close up of the mesh around the ice shape (I-1998-000092). (x3500 Figure 5-a)

\section{Validation of the Pressure Solver}

The validation of the pressure solver has a qualitative and a quantitative component. The qualitative validation investigates the surface pressure distribution over a single analytic hump on a parabola. This parabola resembles the leading-edge of the NACA 0012 airfoil, so the overall geometry can be considered as a simulated roughness element on the leading-edge of the NACA 0012 airfoil. Figure 7 shows the variation of the pressure over the analytic hump as calculated by the in-house solver. There is an increase of pressure at the onset of the hump, and then the pressure drops as the flow accelerates over the hump. There is a pressure plateau under the separation bubble, which is followed by the pressure recovery. For the validation, the study by Beierle et al. ${ }^{22}$ is selected, which was carried out on a NACA 0015 airfoil at zero degree angle-of-attack with an analytic hump. Comparing Figure 7 and findings of Beierle et al. ${ }^{22}$ in Figure 8, the same trends are observed in both results. The difference in the minimum values of the $\mathrm{Cp}$ is due to the higher Reynolds number used in ref. 22.

The second validation is a quantitative one. Since the flow solver is essentially a 2D-DNS solver, there are some limits on higher Reynolds number flows. This limitation is partially alleviated by the use of the ler instead of the chord length of the airfoil for the apparent Reynolds number. Although the literature 


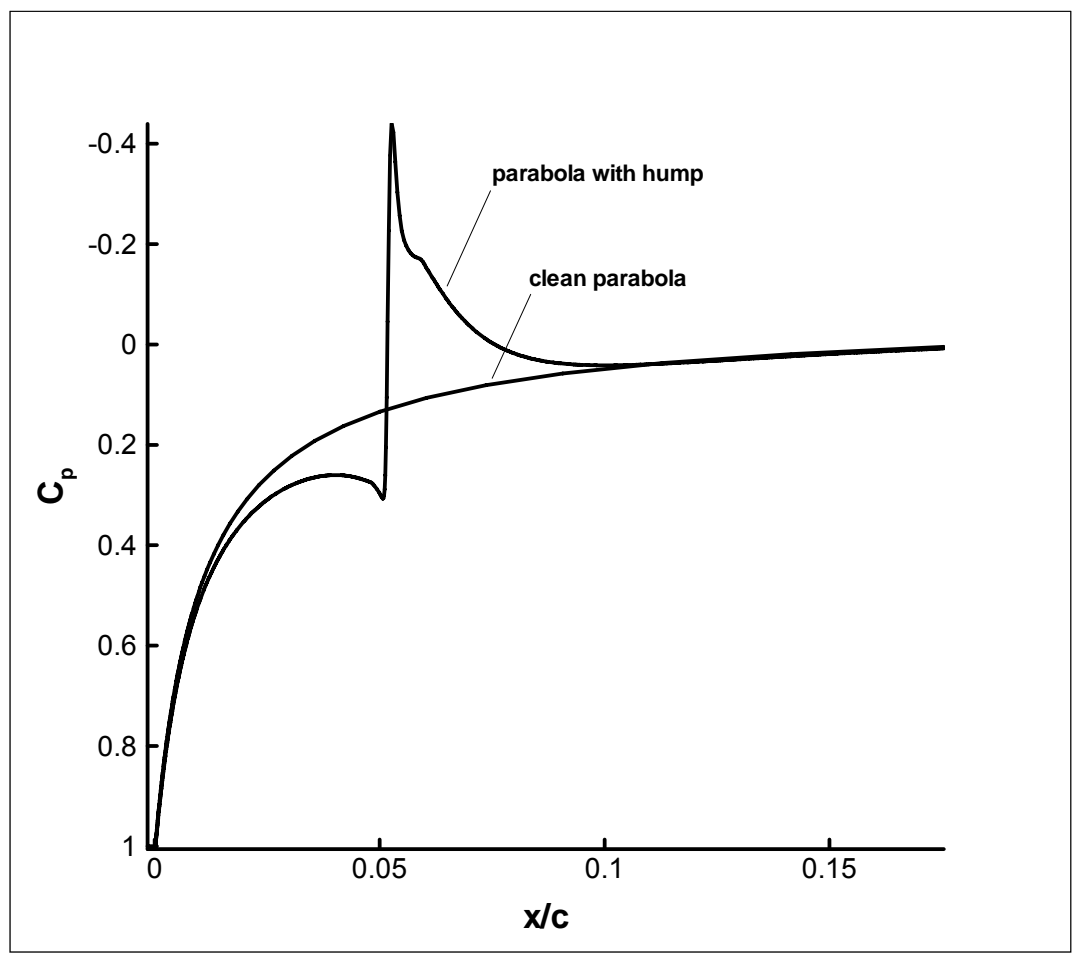

Figure 7. Non-dimensional pressure variation on the analytic hump.

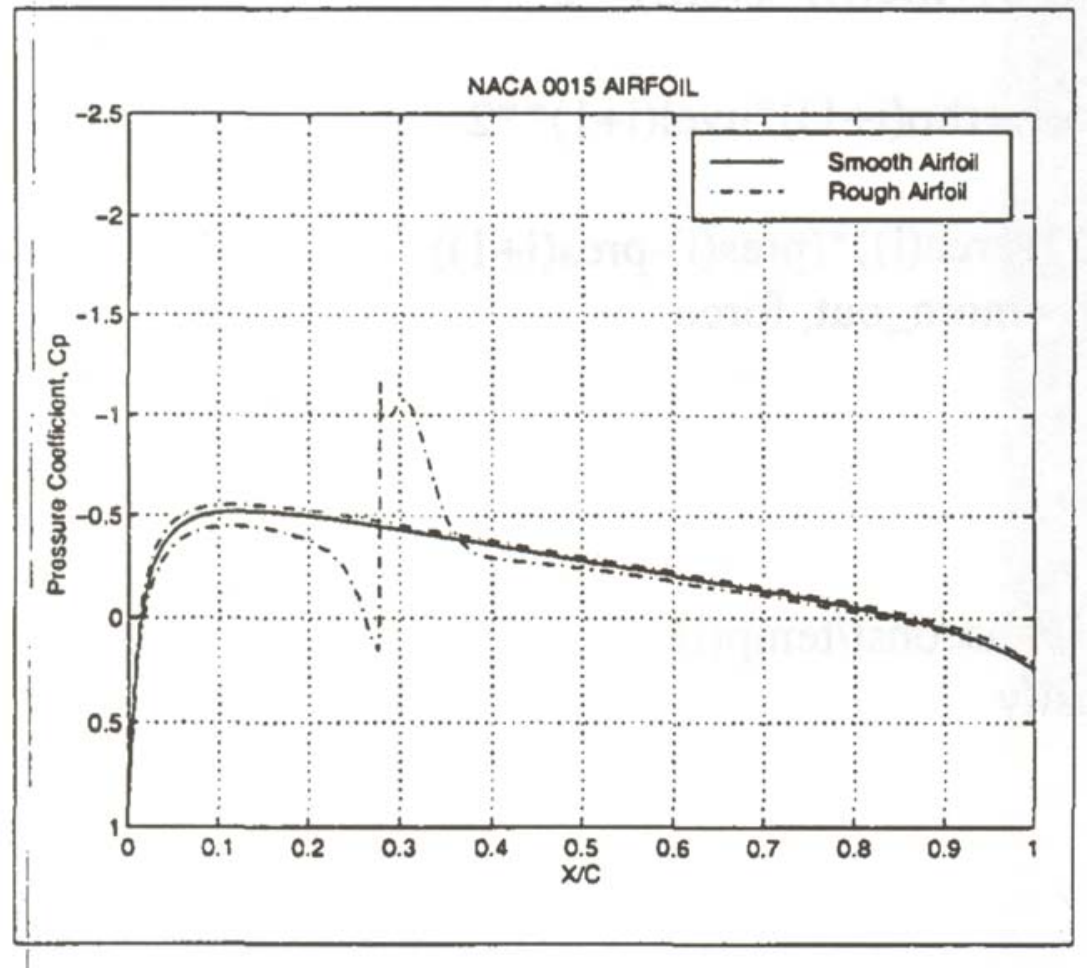

Figure 8. Non-dimensional pressure over a roughness element on a NACA 0015 airfoil, Beierle et al. [22]. 
contains a reasonable amount of $\mathrm{C}_{1}-\mathrm{C}_{\mathrm{d}}$ data for the low Reynolds number airfoils, $\mathrm{C}_{\mathrm{p}}$ distributions for these cases are absent. Therefore, a thorough quantitative validation for this code is also limited.

The study by Raghunathan et al. ${ }^{23}$ presents one such computational study for a NACA 0021 airfoil at several angles of attack all at the same chord Reynolds number of $2.6 \times 10^{5}$ (Figure 9). It is important to note that their pressure data is not validated against other computational or experimental $\mathrm{C}_{\mathrm{p}}$ data, either; they only compared the computed $\mathrm{C}_{1}$ values to other experimental data. The case at 0 degree angle-ofattack was chosen as the benchmark. In Figure 10, the pressure values in the leading-edge region as computed in the present study and the computation of XFOIL code, which is a potential flow solver coupled with a boundary layer solver, for the same region are presented. The surface pressure drops due to the acceleration over the airfoil leading-edge, as seen in both figures. The suction peak is determined at a slightly further downstream location compared to the result of ref. [23] and XFOIL. Although the variation of pressure and the location of the suction peak are captured reasonably well, the values of the current solver are more conservative in terms of the surface pressure values. This is thought to be due to the fact that the Poisson equation has an elliptic nature and is highly dependent on the boundary conditions. In the present study, the downstream boundary condition for an airfoil in air is missing due to the connection to the base parabola. This problem due to the geometry in the downstream locations inherently imposes modifications on the boundary conditions that result in the conservative nature of the calculations. However, the pressure values in the vicinity of the leading-edge are acceptably close to each other. Considering the fact that most icing phenomena occur within this range $(10 \%)$ of the chord, the pressure results therein can be relied on for the ice accretion research purposes.

\section{Flow Field over Complex Ice Roughness}

Two experimental ice shapes were chosen for the pressure field study: one rime ice and one glaze ice on a NACA 0012 airfoil. The handling of the complex ice geometries and the following grid generation was performed with the help of Fourier analysis, as given in ref. 19. In actual experiments, the ice shape that forms over a wing has a spanwise distribution, but a $2 \mathrm{D}$ cross-section is still a good enough representation of the main ice shape. This is because the large-scale features of the ice shape do not vary much over the span, although the small-scale roughness features do vary. Therefore, the flow solution was performed over a 2D cross-section of the experimental ice shape. A snapshot of the resulting unsteady flow over these ice shapes is presented in Figure $11\left(\mathrm{Re}_{\mathrm{c}} \cong 8.2 \times 10^{5}\right)$ for the rime case and in Figure 12 $\left(\mathrm{Re}_{\mathrm{c}} \cong 5 \times 10^{5}\right)$ for the glaze case. It is observed that the presence of the ice roughness causes the flow to form multiple vortices that are shed from the rough surface. As seen in these figures, the whole leadingedge region is subjected to extra low pressure fluctuations induced by the vortices shed from the rough 
surface. The vortex cores are the points where the pressure reaches its minimum values. Vortex-core $C_{p}$ values are as low as -2.26 for rime case and -17.49 for glaze case.

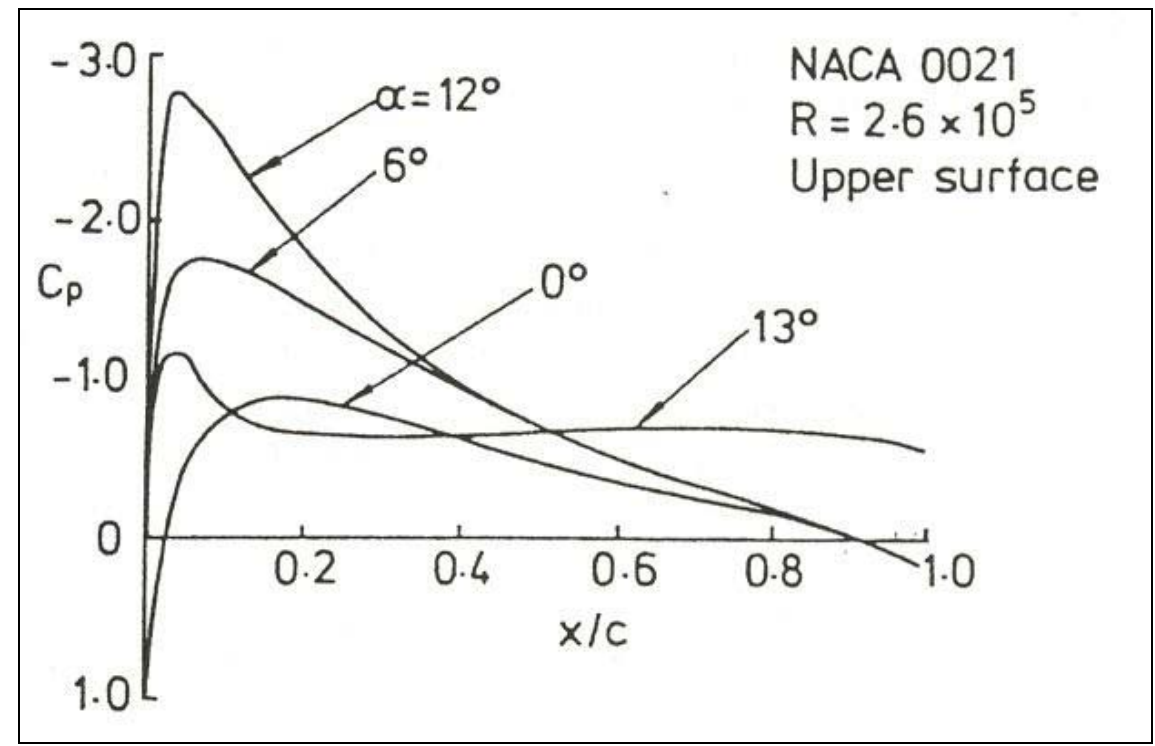

Figure 9. Pressure coefficient on a NACA 0021 airfoil upper surface. Ref [23].

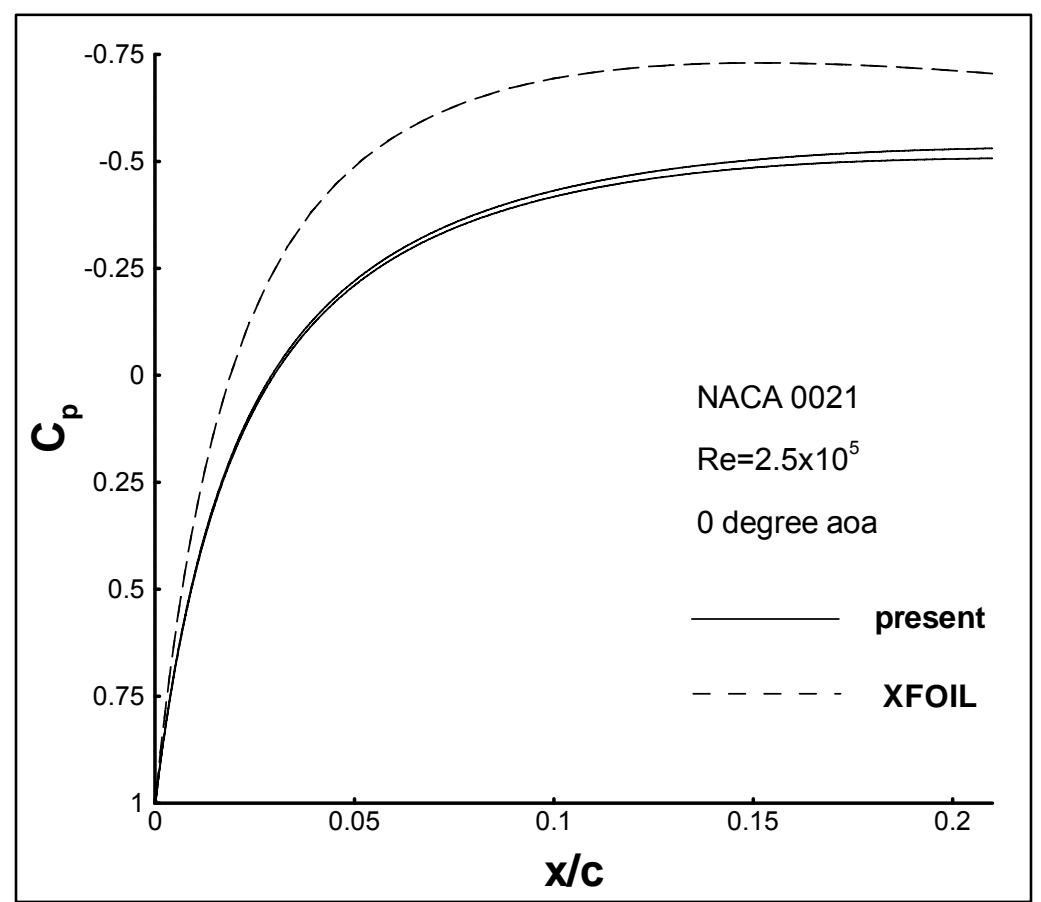

Figure 10. Calculated pressure coefficient distribution on a NACA 0021 leading-edge. 


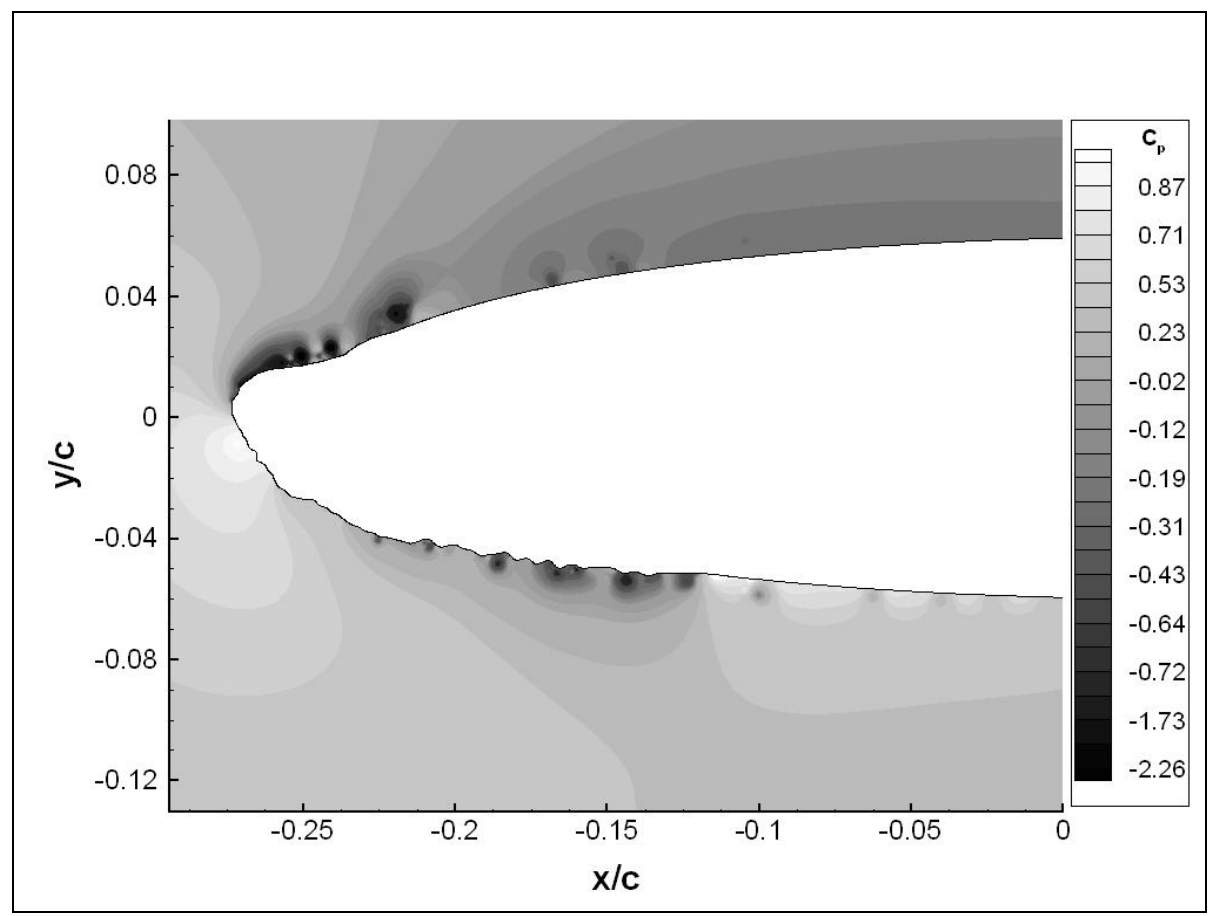

Figure 11. Pressure field in an unsteady flow around experimental rime ice shape ${ }^{13}$ around the NACA 0012.

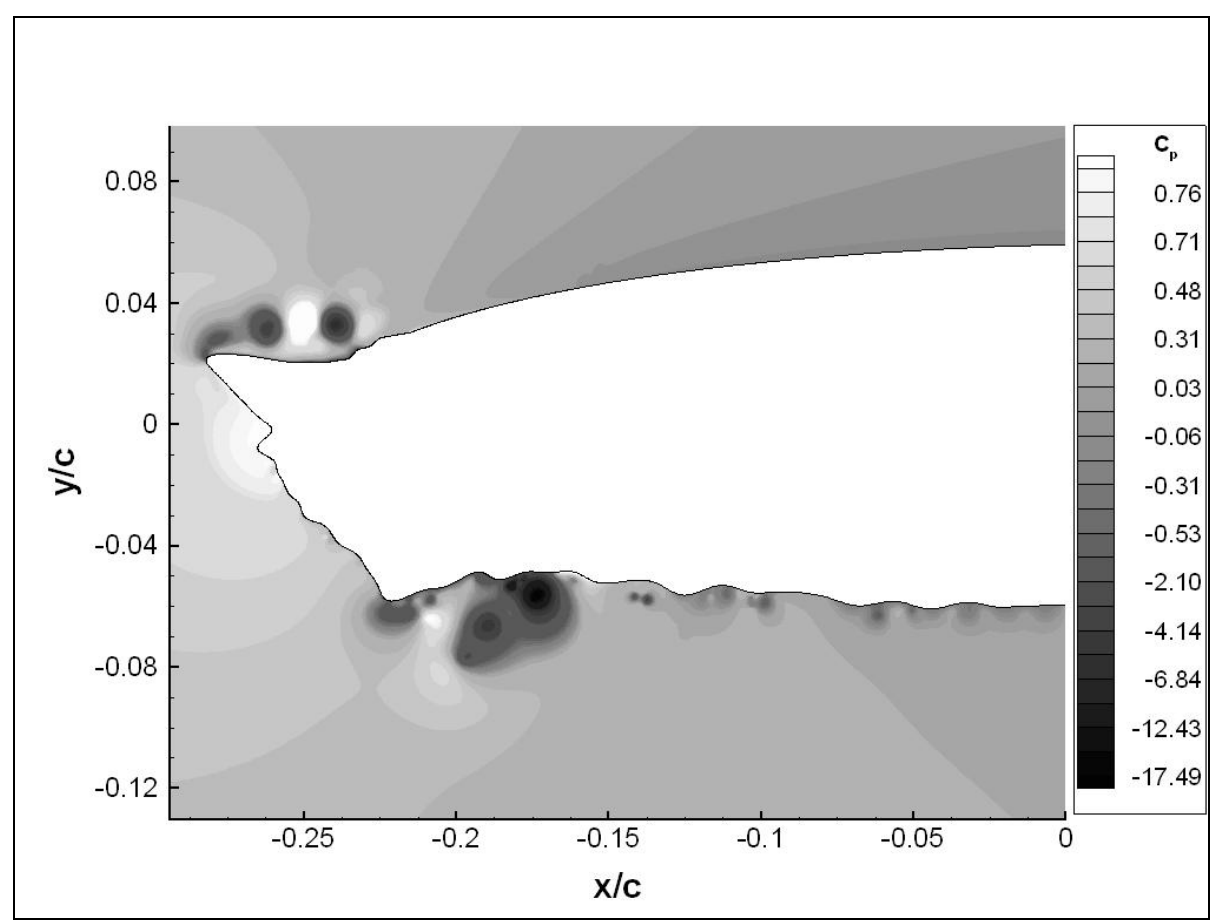

Figure 12. Pressure field in an unsteady flow around experimental glaze ice shape ${ }^{13}$ around the NACA 0012. 


\section{DISCUSSION}

The aim of this study was to derive the relevant equations to investigate the pressure field around airfoil leading-edges with ice roughness and to produce a pressure solver for the resulting Poisson equation. The derivations for the pressure Poisson equation involved a series of transformations, which include a conformal mapping and the Prandtl transposition. The derivation for the wall boundary condition equations involved a series of refinement to avoid the numerical instabilities on top of the abovementioned transformations.

The two major numerical difficulties encountered during the study were the checkerboard instability and the boundary-interior field feedback instability. The checkerboard instability has been taken care of by using a staggered grid, and the feedback instability was taken care of by the derivation of a wall boundary condition that eliminates the need for boundary/interior field coupling.

The numerical results give reliable predictions in terms of the trends within the leading-edge region, but downstream of this region is not as reliable due to conservative behavior of the solver. The vortices shed from the ice roughness are calculated to have low pressure cores that do not dissipate over the leadingedge region and impose fluctuating low pressure on the surface. 


\section{CHAPTER III}

\section{POTENTIAL OF LOCAL RELATIVE HUMIDITY TO AFFECT AIRCRAFT ICING}

This study has been presented at the

AIAA $42^{\text {nd }}$ Aerospace Sciences Meeting \& Exhibit

AIAA paper 2004-0059

\section{INTRODUCTION}

The Messinger model ${ }^{2}$, which connects the ice accretion on the wings to the supercooled water droplets that are impinging on the wing surface, has been actively used by the icing community since it was developed. $\mathrm{LEWICE}^{7}$ is one of the most widely used software tools for ice accretion prediction. Based on the Messinger model, LEWICE can produce excellent predictions for the rime ice conditions, where the ambient temperature is well below the freezing point and the droplets freeze at impact with the wing leading-edge surface. But for the glaze ice conditions, where the temperature is only slightly below the freezing point, the model has more difficulty in making acceptable predictions (see Figure 13-a). One other area where this model can experience problems is ice growth downstream of the primary ice shape, which can be typical ice, feather growth, or frost formation. An example of this is given in Figure 13-b, which shows the experimental ice accretion on an airfoil along with the LEWICE prediction ${ }^{13}$.

Some potential reasons for these discrepancies in the icing model compared to experiment and the ways to alleviate them have been investigated. Olsen and Walker ${ }^{10}$ state that the water film, which is supposed to cause the downstream growth of the ice was not observed in their experimental study, other than during some initial moments of the ice accretion. This shows that in order to better explain the downstream transport of the water and growth of ice, new mechanisms need to be incorporated.

With the help of other experimental evidence, the aspects of the icing physics that the Messinger model is missing have been investigated. These aspects include the splashing and breaking of the droplets, accounting for the effect of ice roughness on the boundary layer development and on the heat transfer, working on the motion and growth of the water droplets on the surface, and studying the water film dynamics due to ice roughness-air flow interaction ${ }^{4}$. These details are expected to better determine the water distribution over the surface and the consequent changes in the heat transfer process ${ }^{11}$. 


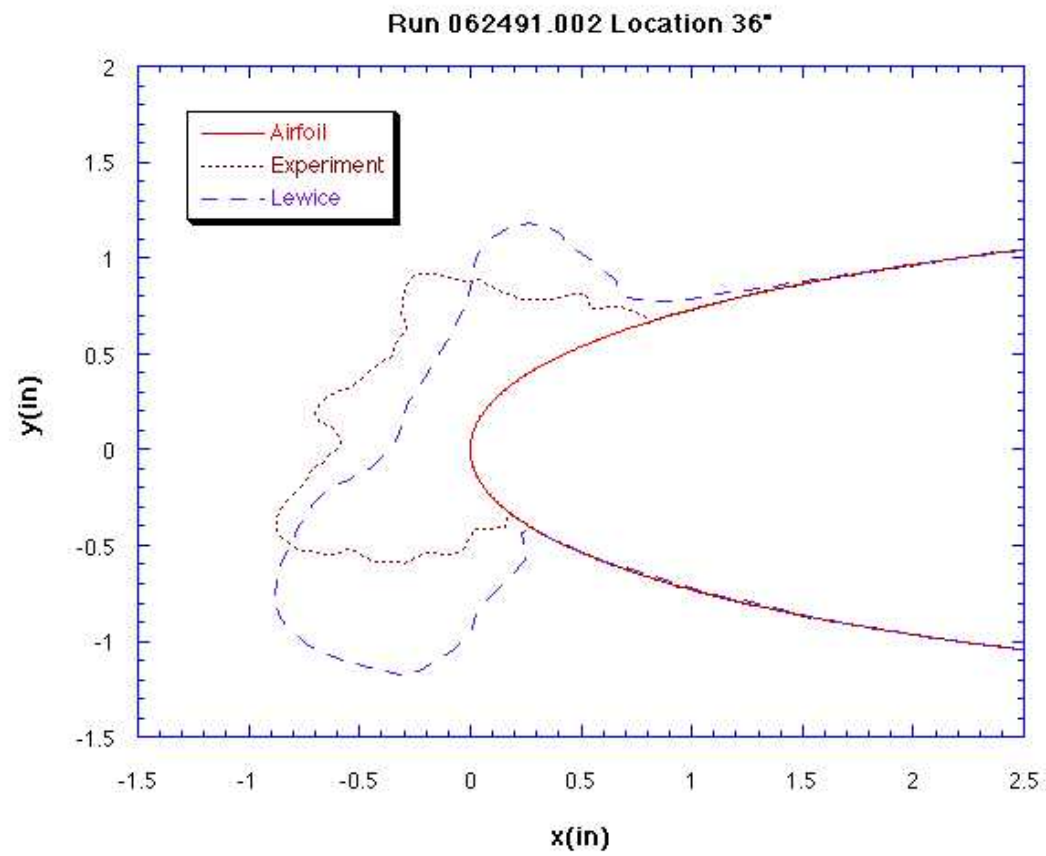

a) glaze ice large scale

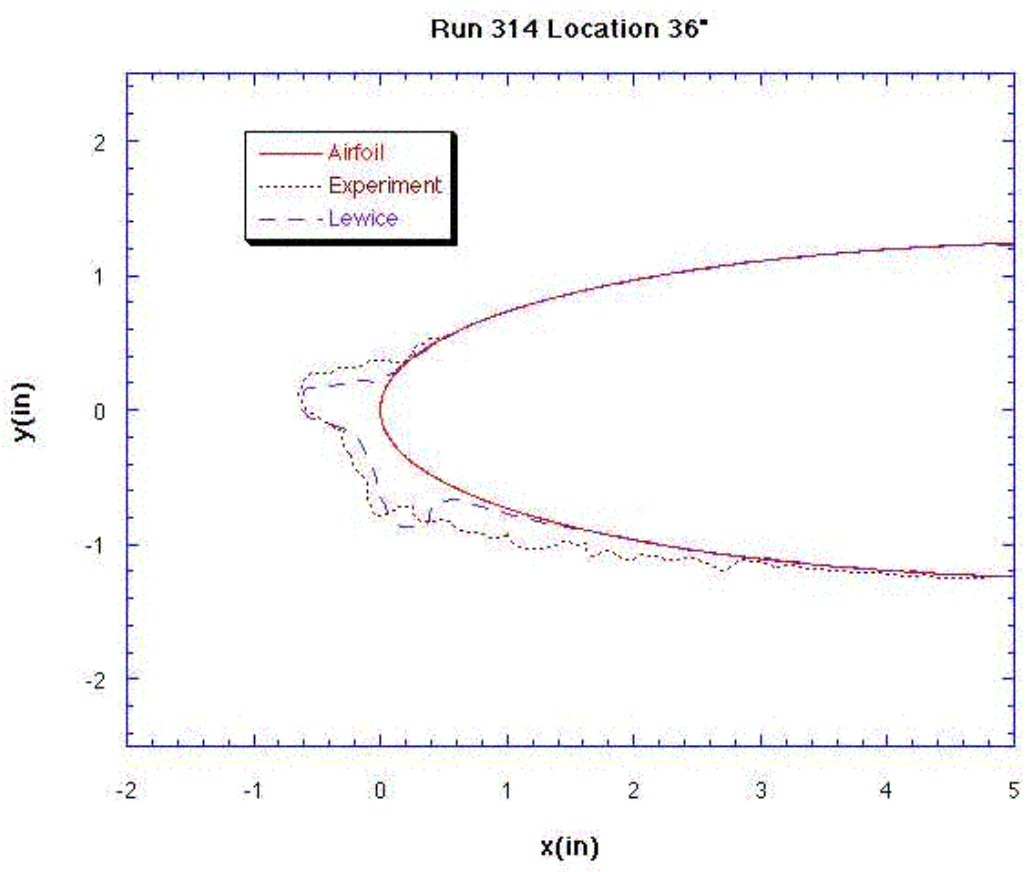

b) small scale ice and downstream frost

Figure 13. Experimental ice accretion on an airfoil and the corresponding LEWICE prediction, Ref. [13]. 
The present work investigates whether one other parameter should be considered in icing simulations: local relative humidity effects. From the LEWICE 2.0 user's manual ${ }^{7}$ : “... since relative humidity is at best a secondary effect on the ice accretion process, a value of $100 \%$ can be assumed." The present author agrees that relative humidity is a secondary effect for the primary ice shape, particularly in the impingement region. However, it is potentially a first-order effect for several other modes of ice growth (downstream frost, feather, small scale ice) downstream of this region.

Depending on the actual flight and local atmospheric conditions, a drop in pressure may lead to supersaturation and an increase in pressure may lead to subsaturation. So the stagnation region can induce droplet evaporation due to subsaturation, whereas the suction or low pressure regions may be exposed to condensation/deposition or may cause droplet growth due to supersaturation. If the condensation and/or deposition occur onto a surface, the result will be droplets or a frost formation. However, in the case of a shed or entrapped vortex, the low pressure at the core can produce a supersaturated region, which produces favorable conditions for the growth of an entrained droplet that can be deposited as it becomes larger. This result agrees with the idea that the roughness itself can play a key role in its own growth through the interaction with the surrounding flow.

The relative humidity can impact the accretion process locally provided that there is a subsaturation or a supersaturation and a sufficient time allowance. Subsaturation or supersaturation can be formed by local changes of pressure in the flow field. A pressure drop, for example, can cause localized supersaturation in saturated ambient conditions. There are several instances of this phenomenon in fluids applications such as condensation at high angle of attack conditions of the fighter aircraft, condensation in the low-pressure cores of the tornados.

The last example is a case in which vortex dynamics and supersaturation due to drop in pressure contribute to condensation. The investigation of the flow around airfoils with ice accretion has shown that the ice roughness, especially glaze ice, can induce a large separation region. Studies by Bragg et al. ${ }^{24}$ and Dunn et al. ${ }^{16}$ are good examples of flow simulations around airfoils with glaze ice. They both show a steady separation vortex behind the horn of the glaze ice. The surface pressure graphs given in the mentioned papers show the low surface pressure plateau along the separation bubble. Pan et al. ${ }^{18}$, in agreement with the findings of Dunn et al. ${ }^{16}$, found that the ice causes a low pressure plateau under the separation vortex and that the ice leads to a decrease in the maximum suction over the airfoil. Gurbacki and Bragg $^{25}$ worked on an experimental glaze ice on a NACA 0012 airfoil, which shows the decrease in the maximum suction and the low pressure plateau behind the ice horn. They also show that as the angle of attack increases, the suction peak increases despite the ice, and at the same time the rear separation 
bubble becomes larger. Bencic ${ }^{26}$ and Ferrigno et al. ${ }^{27}$ made use of pressure sensitive paint to measure the surface pressure variation around airfoils with both rime and glaze ice. Their experimental studies show pressure coefficient values as low as -6.0 to -7.0 aft of the ice horns. So it can be inferred that the vortex shedding from the rough surface of the ice or the vortices entrapped within the ice roughness have the potential to condense the humidity in the air either on the surface or on the local droplets.

The present study is composed of two main parts. The first part is a parametric study of the variations of the saturation levels in the flow field over a simulated roughness element on a parabola surface. Investigations of the flow field over experimental ice accretions on a NACA 0012 airfoil $^{13}$ (see Figure 1 and Figure 14) are included in the second part to have a feel of the realistic cases. For the parametric

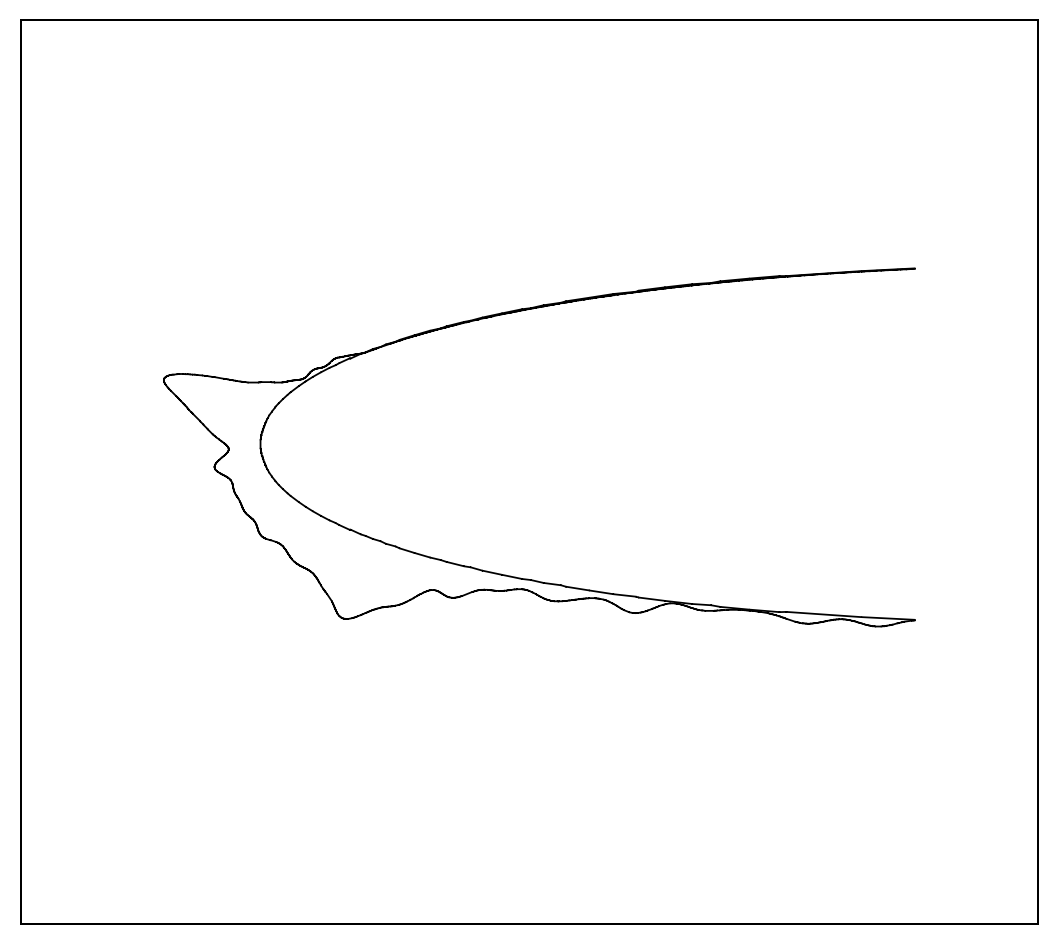

Figure 14. NACA 0012 leading-edge and the experimental glaze ice shape ${ }^{13}$.

study in the first part, the contributions of the following parameters to the change of saturation levels were studied: roughness size and the Reynolds number, the ambient relative humidity, and the angle of attack. The assumptions made in this analysis are:

- Incompressible flow,

- Temperature calculated from the isentropic relation,

- Saturation with respect to liquid water and with respect to ice are considered, and 
- The only phase that is considered is vapor and there are no phase changes: the simulation only looks at the changes in relative humidity and shows regions of the flow field that will be susceptible to condensation/deposition or evaporation. This assumption also entails that the total water content of the air is constant and is in the vapor form only.

\section{GOVERNING EQUATIONS}

The flows considered in this study are incompressible, and the flow solver is a DNS-type solver with $2 \mathrm{D}$ constraint. So, the code has the capability to resolve all the details of the flow over the geometry as long as $3 \mathrm{D}$ effects are negligible. The formulation of the solver is in terms of stream function and vorticity. This type of formulation eliminates the effort of dealing with pressure during the iterations for the flow. The flow field equations that are numerically solved are:

$$
\begin{aligned}
& \bar{\xi}_{\xi \xi} \Psi_{\bar{\xi}}+\left(\bar{\xi}_{\xi}\right)^{2} \Psi_{\bar{\xi} \bar{\xi}}-f_{\xi \xi} \bar{\eta}_{\eta} \Psi_{\bar{\eta}}-2 f_{\xi} \bar{\xi}_{\xi} \bar{\eta}_{\eta} \Psi_{\bar{\xi} \bar{\eta}}+ \\
& \left(1+\left(f_{\xi}\right)^{2}\right) \eta_{\eta \eta} \Psi_{\bar{\eta}}+\left(1+\left(f_{\xi}\right)^{2}\right)\left(\bar{\eta}_{\eta}\right)^{2} \Psi_{\bar{\eta} \bar{\eta}}=\left(-h^{2}\right) \Omega+\Psi_{\tau} \\
& \Omega_{\tau}+h^{2} \Omega_{t}+\bar{\xi}_{\xi} \bar{\eta}_{\eta}\left(\Psi_{\bar{\eta}} \Omega_{\bar{\xi}}-\Psi_{\bar{\xi}} \Omega_{\bar{\eta}}\right)+\left(\psi_{i n v}\right)_{\eta} \bar{\xi}_{\xi} \Omega_{\bar{\xi}}-f_{\xi}\left(\psi_{i n v}\right)_{\eta} \bar{\eta}_{\eta} \Omega_{\bar{\eta}}- \\
& \left(\psi_{i n v}\right)_{\xi} \bar{\eta}_{\eta} \Omega_{\bar{\eta}}=\operatorname{Re}^{-1}\left[\bar{\xi}_{\xi \xi} \Omega_{\bar{\xi}}+\left(\bar{\xi}_{\xi}\right)^{2} \Omega_{\bar{\xi} \bar{\xi}}-f_{\xi \xi} \bar{\eta}_{\eta} \Omega_{\bar{\eta}}-2 f_{\xi} \bar{\xi}_{\xi} \bar{\eta}_{\eta} \Omega_{\bar{\xi} \bar{\eta}}+\right. \\
& \left.\left(1+\left(f_{\xi}\right)^{2}\right) \bar{\eta}_{\eta \eta} \Omega_{\bar{\eta}}+\left(1+\left(f_{\xi}\right)^{2}\right)\left(\bar{\eta}_{\eta}\right)^{2} \Omega_{\bar{\eta} \bar{\eta}}\right]
\end{aligned}
$$

A thorough explanation of the flow solver has been given in references [14] and [19].

Since the pressure is not solved as a part of the flow, it has to be post-processed after obtaining a converged flow field. The pressure solver has been explained in detail in chapter II, or in ref. 28. The final equation that is solved numerically is:

$$
C_{p_{\xi \xi}}+\left(1+f_{\xi}^{2}\right) C_{p_{\eta \eta}}-f_{\xi \xi} C_{p_{\eta}}-2 f_{\xi} C_{p_{\xi \eta}}=R
$$

where,

$$
R=4\left[\left(u_{\xi}-f_{\xi} u_{\eta}+\frac{v(\eta+f)}{\xi^{2}+(\eta+f)^{2}}\right)\left(v_{\eta}+\frac{u \xi}{\xi^{2}+(\eta+f)^{2}}\right)-\left(v_{\xi}-f_{\xi} v_{\eta}-\frac{u \eta}{\xi^{2}+(\eta+f)^{2}}\right)\left(u_{\eta}-\frac{v \xi}{\xi^{2}+(\eta+f)^{2}}\right)\right]
$$

In accordance with the pressure solver, the pressure values that are given in this paper are in nondimensional form according to the standard pressure coefficient $\left(\mathrm{C}_{\mathrm{p}}\right)$ relation: 


$$
C_{p}=\left(P-P_{\infty}\right) /\left(\frac{1}{2} \rho_{\infty} V_{\infty}^{2}\right)
$$

\section{Numerical Method}

The implicit flow solver uses a bi-directional sweep scheme which composes one "pseudo-time iteration." The solver iterates in pseudo time until a specified level of convergence is achieved. In case of an unsteady flow, every physical time step consists of pseudo time sub-iterations which continue until convergence or a specified number of iterations. The pressure solver uses a Gauss-Siedel iteration scheme out to a specified convergence level. For the surface pressure (i.e., wall boundary condition), an analytic expression is used which gives the wall pressure values directly from the converged flow parameters $(\psi-$ $\omega)$. Thus the surface pressure values are solved separately before the interior field iterations start. For all discretization in space and time, second order accuracy is used. The absolute convergence rate for the flow variables (i.e., $\Psi, \Omega, C_{p}$ ) can be as low as $10^{-4}$ or $10^{-5}$ depending on the complexity of the surface roughness, grid size and the available computation time. Studies were performed to confirm the independence of the grid, time step, and convergence level for the simulations of this study.

\section{Calculation of Relative Humidity}

There are two main parameters that determine the level of relative humidity (i.e., saturation level): the amount of water vapor present in the air and the maximum amount of vapor that air can hold at the local saturation conditions. Since there are two kinds of saturation, one with respect to liquid water and one with respect to ice, relative humidity is also defined in similar fashion: relative humidity with respect to liquid water $\left(R H_{w}\right)$, and relative humidity with respect to ice $\left(R H_{i c e}\right)$. In terms of $R H_{w}$, a supersaturation will cause condensation to liquid phase, whereas a subsaturation will favor evaporation to vapor phase. Similarly in case of $R H_{i c e}$, a supersaturation will cause deposition to solid phase, whereas a subsaturation will cause sublimation to gas phase. It should be noted that even if the ambient conditions are subsaturated with respect to liquid water, there still could be supersaturation with respect to ice. However, it is also likely that a supersaturation with respect to both liquid water and ice are present in the flow field. In this case, the effects of both saturations will be observed; but condensation will be the faster compared to deposition.

In the present study, the potential for condensation/deposition due to supersaturation through interaction with the flow field, thus the potential for additional ice growth beyond the direct impingement mechanism, is investigated as a function of several parameters. This means that the actual phase change between water vapor and liquid water or ice is not considered, only the potential for such change is 
investigated. This assumption requires a constant amount of total vapor in the flow field. Along with the incompressible assumption for the governing flow, a constant vapor amount results in a constant mixing ratio, $w$, which is defined as the ratio of the vapor density to the density of the dry air. The mixing ratio is going to be employed to calculate the variation of the vapor pressure, as outlined later in this chapter:

$$
w=\rho_{v} / \rho_{d}
$$

where $w$ is the mixing ratio, $\rho_{v}$ is the vapor density, and $\rho_{d}$ is the density of the dry air ${ }^{29}$. Since the present study assumes incompressible flow, the density of dry air is constant. Taking into account the assumptions of no phase change, which requires $\rho_{v}=$ constant, it can be concluded that the mixing ratio, $w$, is constant.

As the fluid travels through the flow field, the static pressure changes locally, which in turn alters both the vapor pressure, $e$, and the saturation vapor pressure $\left(e^{*}\right.$ or $\left.e^{\#}\right)$. The changes in saturation levels are determined by keeping track of these two parameters: the saturation vapor pressure (hence maximum amount of vapor air can hold at saturation conditions) and the actual vapor pressure. The partial pressure of vapor $(e)$ is directly proportional to the amount of vapor in the air. The relation between the vapor pressure and the vapor density is obtained by making use of the mixing ratio, $w$.

$$
w=0.622 \frac{e}{p-e}
$$

where $p$ is the local static pressure ${ }^{29}$. Since the local pressure changes in the flow field, so does the value of the vapor pressure in order to keep the mixing ratio constant.

Another factor that contributes to the change of relative humidity is the change of saturation vapor pressure, which depends on the local temperature. The isentropic relation is employed to obtain the temperature field from the pressure field.

$$
\left(\frac{T}{T_{\infty}}\right)=\left(\frac{P}{P_{\infty}}\right)^{\frac{\gamma-1}{\gamma}}
$$

In Eq. 37, $\gamma$ is the ratio of the specific heats for dry air. A legitimate concern at this point is that the isentropic relation accounts for changes in density, whereas the flow solver employed in this study is an incompressible one. So how are these two justified? The approach utilized at this point is similar to Boussinesq approximation. Although the flow is incompressible, for severe changes in pressure, the difference between the incompressible results and the isentropic results will be enough to cause high level 
of error in the relative humidity calculations. Figure 15 exemplifies the growth of error in the calculation of relative humidity as a function of drop in pressure. For a $C_{p}$ value of -3 , there is almost $100 \%$ difference between the two results. This is because very small changes in density can store or release much energy that can cause significant change in saturation level. This behavior of saturation level is implied by its exponential change as a function of temperature. As a solution at this point, the isentropic relation is used for the calculation of temperature from the pressure field.

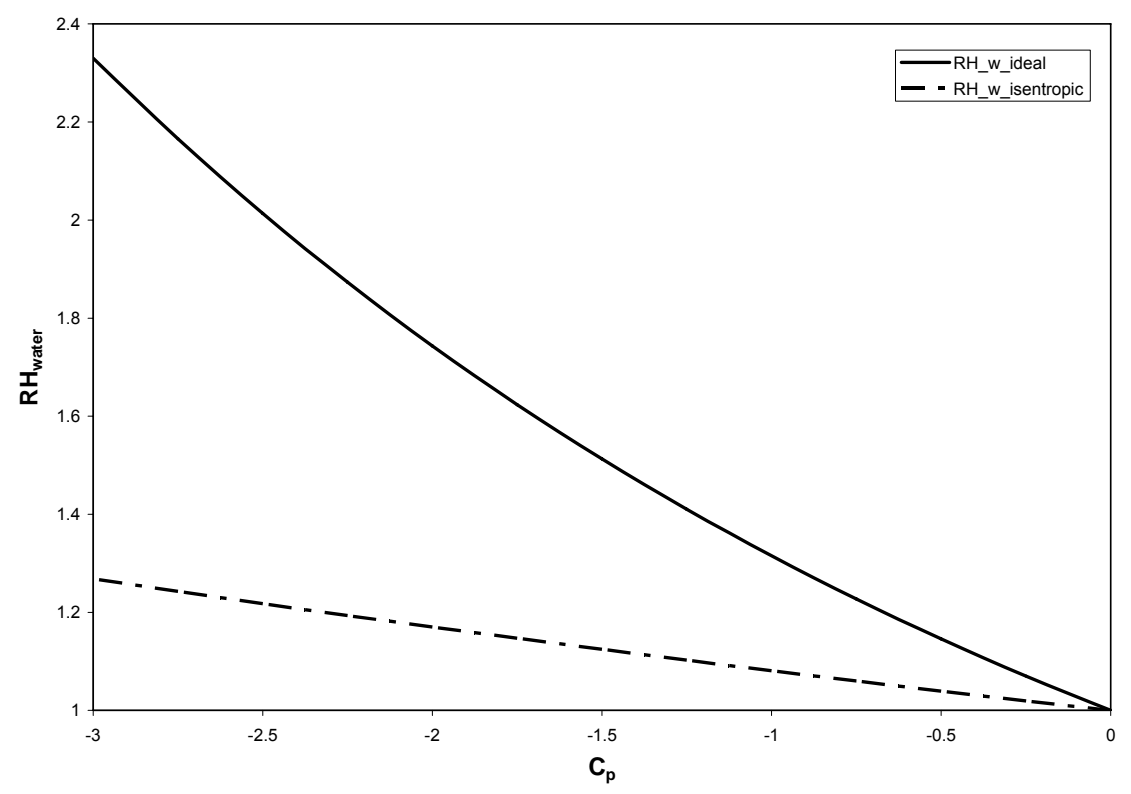

Figure 15. Comparison of variation of relative humidity $(R H)$ as given by ideal gas and isentropic relations; values are calculated for 21 inch chord length and $5000 \mathrm{~m}$ altitude in standard atmosphere;

$$
R e_{c}=1 \times 10^{6} .
$$

This temperature field is then fed into the below formulae to obtain the variation of saturation vapor pressure with respect to liquid water $\left(e^{*}\right)$ and with respect to ice $\left(e^{\#}\right)$, respectively.

$$
e^{*}=6.112 \exp (17.67 T /(T+243.5))
$$

where $e^{*}$ is in millibars and temperature in Celsius ${ }^{29}$,

$$
e^{\#}=\exp \left(23.33086-\frac{6111.72784}{T}+0.15215 \ln (T)\right)
$$

where $e^{\#}$ is in millibars and temperature in Kelvin ${ }^{29}$. 
It is important to note at this point that the relative humidity with respect to liquid water and ice are not the same since the saturation with respect to liquid water and saturation with respect to ice are not generally equal to each other. Local relative humidity at a location can be defined as the ratio of the actual vapor pressure $(e)$ to the vapor pressure at local saturation conditions $\left(e^{*}\right.$ or $\left.e^{\#}\right)$. When this ratio, i.e., relative humidity, is equal to 1 , it is said that the gas is saturated with respect to liquid water or ice. If this value is less than 1 , it is subsaturated, and if it is greater than 1, it is supersaturated. For example, if the vapor pressure ratio turns out to be 1.1 , then the gas is at a relative humidity of $110 \%$ and a supersaturation of $10 \%$.

The ambient relative humidity in the approaching flow is an input parameter for the calculations. Here, for the sake of explanation of the formulae that are used, the relative humidity in the approaching flow is assumed as $100 \%$ with respect to liquid water in accordance with the literature ${ }^{7}$. Therefore, in the upstream flow, the vapor pressure $\left(e_{\infty}\right)$ is equal to the saturation vapor pressure with respect to liquid water $\left(e^{*}\right)$. Through Equation 36, the value of the mixing ratio of the approaching flow can be obtained, which is constant all along the flow field. So using the mixing ratio, $w$, the local pressure, $p$, and Equation 36 one can calculate the variation of the vapor pressure, $e$, in the flow field. The value of the local saturation vapor pressure $\left(e^{*}\right.$ or $\left.e^{\#}\right)$ is obtained through Equations 38 and 39. Having obtained the local vapor pressure and the local saturation vapor pressure, the local relative humidity is calculated as outlined below.

1.Using Equation 38 and the local altitude information, get $e_{\infty}^{*}$

2.Ambient vapor pressure is:

$$
e_{\infty}=R H_{w} e_{\infty}^{*}
$$

3. Since mixing ratio is assumed as constant,

$$
w=w_{\infty}=0.622 \frac{e_{\infty}}{p_{\infty}-e_{\infty}}
$$

4.Using the local static pressure and the mixing ratio, get the local vapor pressure,

$$
e=(w p) /(0.622+w)
$$

5.Calculate the local temperature using the isentropic relation (Equation. 37) with the calculated local pressure.

6.Calculate $e^{*}$ and $e^{\#}$ using Equations 38, 39 and the local temperature,

7.The local relative humidity values are:

$$
R H_{w}=e / e^{*} \quad R H_{i c e}=e / e^{\#}
$$




\section{RESULTS}

As an introduction to the results of the present study, it is useful to see graphically the relation between the pressure coefficient and the relative humidity. This will give a sense of the strong potential for the supersaturation effects as the pressure and relative humidity change locally within the leadingedge region. Using the assumptions and the methodology outlined in the governing equations section, the variation of relative humidity versus non-dimensional pressure is plotted in Figure 16. This graph is obtained for a flight altitude of $5 \mathrm{~km}(\approx 16400 \mathrm{ft}, \mathrm{T}=255.7 \mathrm{~K}, \mathrm{P}=54440 \mathrm{~Pa})$, and a flight speed of $71 \mathrm{~m} / \mathrm{s}$ (160 mph). The reason for choosing this altitude is that it is a representative flight altitude for many small aircraft that are vulnerable to icing hazards due to their low power supply. The corresponding Reynolds number for this case is $1.7 \times 10^{6}$ based on a chord length of 21 inches, which is the typical chord length used in the experiments. The first thing to notice in Figure 16 is that $R H_{i c e}$ is always at a higher level than $R H_{w}$. Therefore, in the computations of the present study, since the ambient air is assumed at a relative humidity of $100 \%$ with respect to liquid water, it is already supersaturated with respect to ice. Figure 16 also shows that, if no condensation or deposition is allowed, as the pressure drops, high supersaturation levels can easily be obtained especially with respect to ice, though any level of supersaturation will be a favorable environment for condensation and/or deposition.

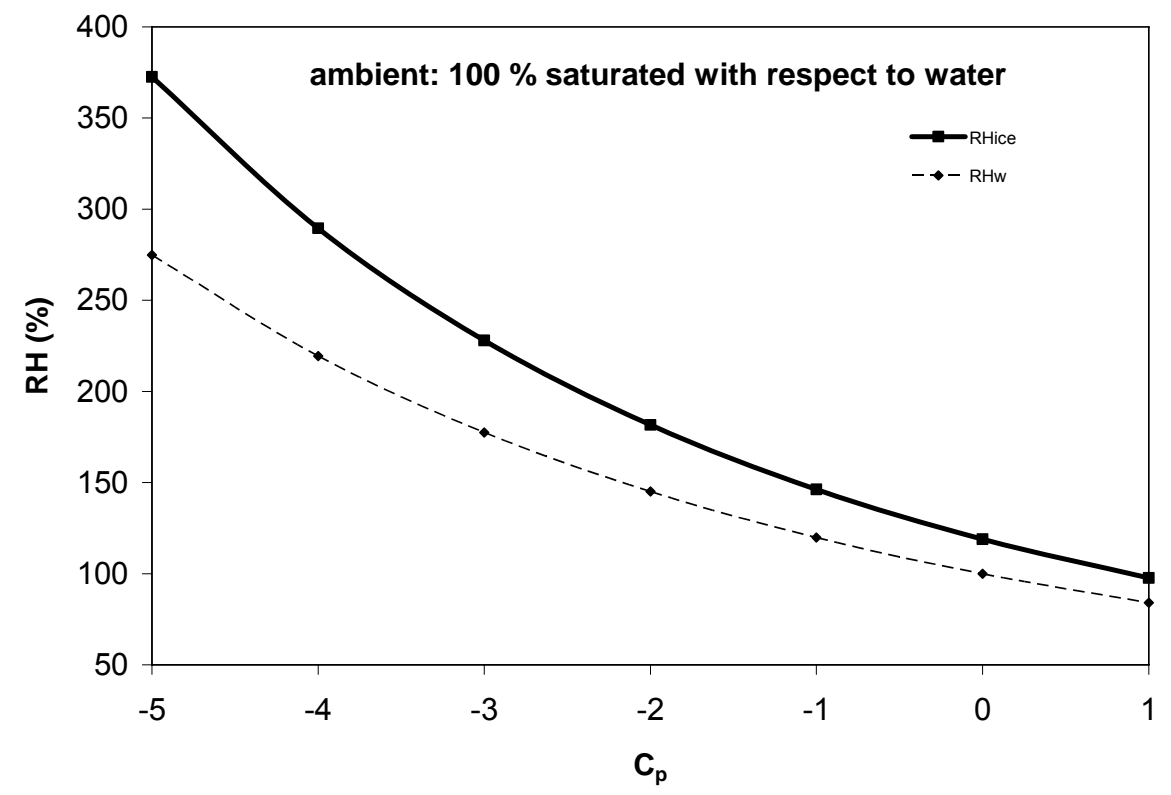

Figure 16. Variation of relative humidity $(R H)$ as a function of $C_{p}$ at $5000 \mathrm{~m}$ altitude in standard atmosphere; $R e_{c}=1.7 \times 10^{6}$. 
According to reference 30, the highest probable value of $R H_{w}$ in free atmosphere is $101 \%$ provided that there is sufficient number of condensation nuclei present in the air, on which vapor can condense. In icing applications, either the available particles in the atmosphere or the already formed droplets with various radii provide these nucleation sites. Bearing this in mind and looking at the numbers given in Figure 16, the strong potential for supersaturation effects can easily be seen. These high values of relative humidity can only be obtained if there is no condensation or deposition. However, in a real case there is condensation and deposition as the air goes through the flow field. It is also useful to note that if, on the other hand, the ambient relative humidity is less than $100 \%$, it will favor the vapor form of water due to evaporation and/or sublimation. This kind of condition would also cause a decrease in the condensation effects over the low-pressure regions.

The first part of the results of the present study consists of the effects of various parameters on local pressure, hence supersaturation, variation in the flow field, along with a discussion of the assumptions involved. The parameters that have been investigated include: size of the roughness element, Reynolds number, ambient relative humidity, and angle of attack. The roughness element that is used is a single analytic hump of varying height. Finally, in the second part of the results section, a discussion of the flow field over an experimental ice shape is presented.

\section{Effect of Size and Reynolds Number}

In order to visualize the effect of roughness size, three humps with different sizes were simulated in separate identical flows where Reynolds number based on the leading-edge radius $\left(R e_{l e r}\right)$ is 100 (corresponding Reynolds number based on chord length $\left(R e_{c}\right)$ is 6300). The reason for choosing this low Reynolds number is to be able to single out the effects of size and not interfere with potential turbulence

effects. The non-dimensional sizes of the humps $(h / c)$ were $1.13 \times 10^{-3}, 2.26 \times 10^{-3}$, and $4.52 \times 10^{-3}$. The corresponding surface pressure variation is shown in Figure 17 along with an example $\psi$ contour plot. The $C_{p}$ values are plotted against $x$, which is the x values non-dimensionalized by the leading-edge radius. Figure 17 shows that a larger roughness element is associated with a larger pressure drop not only on its surface but also in its wake. As discussed earlier, lower pressure is associated with higher supersaturation. Therefore, since the larger hump induces a lower pressure region, it is more susceptible to condensation and deposition.

To investigate the Reynolds number effects, two other simulations were performed: one at $R e_{l e r}=5000$ and one at $R e_{l e r}=10000$. Figure 18 shows the resulting vortex shedding over the roughness element at $R e_{l e r}=5000\left(R e_{c} \cong 3.15 \times 10^{5}\right)$ at standard sea level (SSL) conditions. As seen here, the increase in Reynolds number causes the aft separation bubbles to breakdown and be shed in a time dependent fashion. The 
resultant pressure distribution at a snapshot in the field is given in Figure 19. The shed vortices not only impose a lower surface pressure, but also have low-pressure cores which increase the local supersaturation levels. By looking at the vortex cores, it is seen that $C_{p}$ values as low as -2.32 are obtained, which continue to decrease as the vortex separates from the roughness element and accelerates thereof.
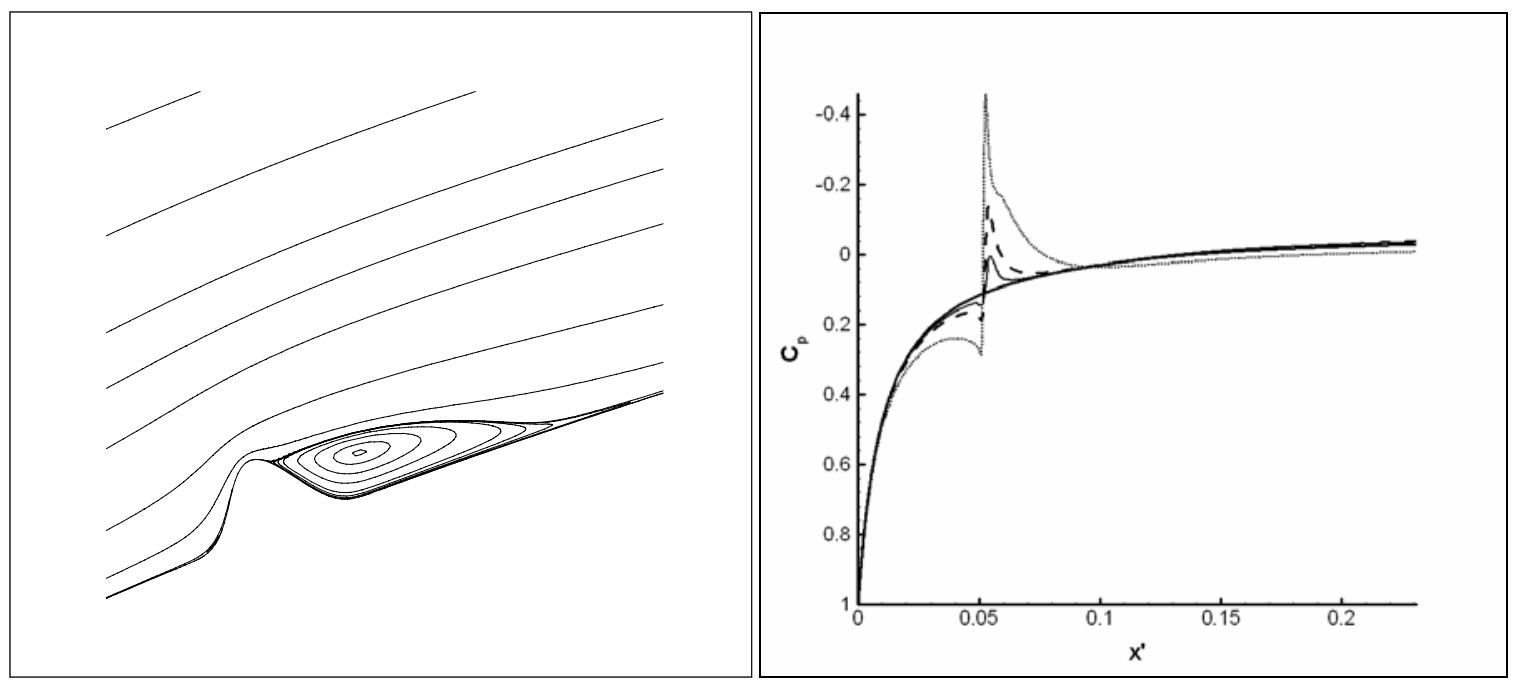

Figure 17. Stream function contours around a hump and the comparison of the surface pressure on humps with different sizes. $R e_{l e r}=100$.
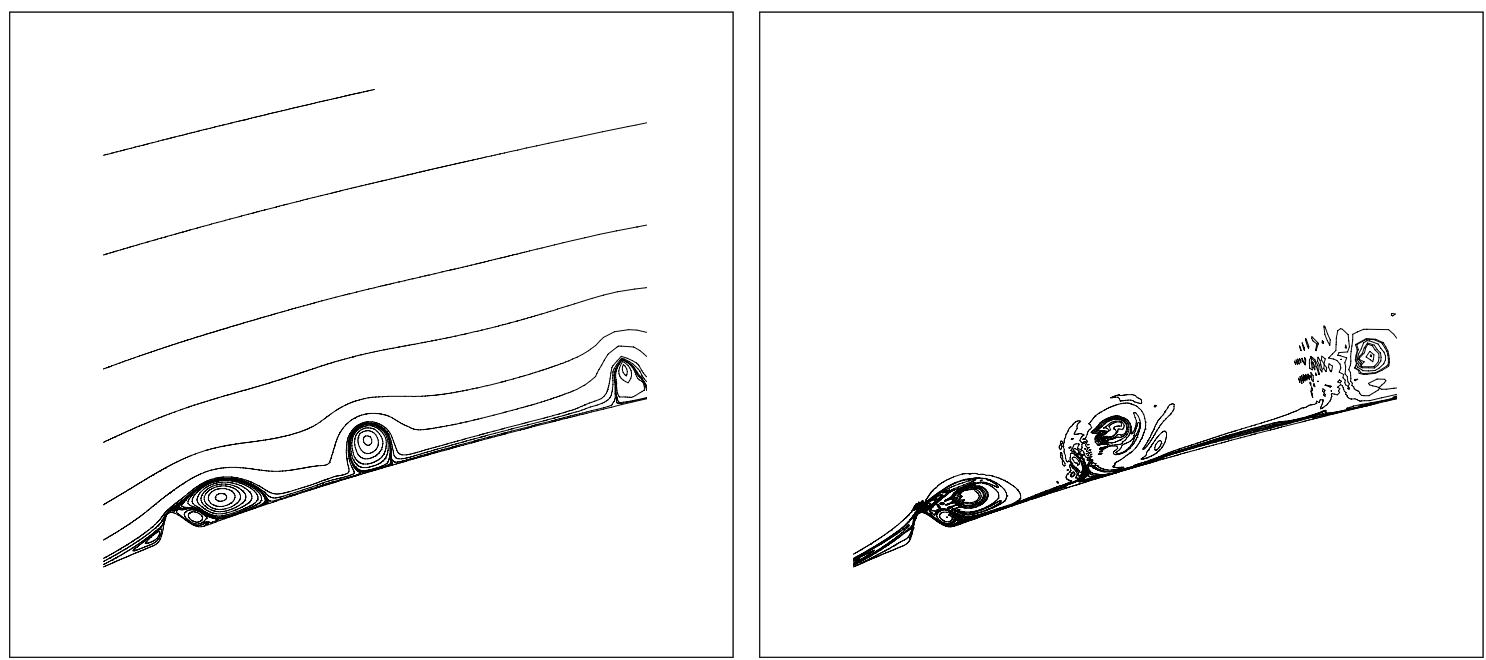

Figure 18. A snapshot from the unsteady flow: stream function $(\psi)$ and the vorticiy $(\omega)$ contours around a simulated roughness element. $R e_{l e r}=5000$. 

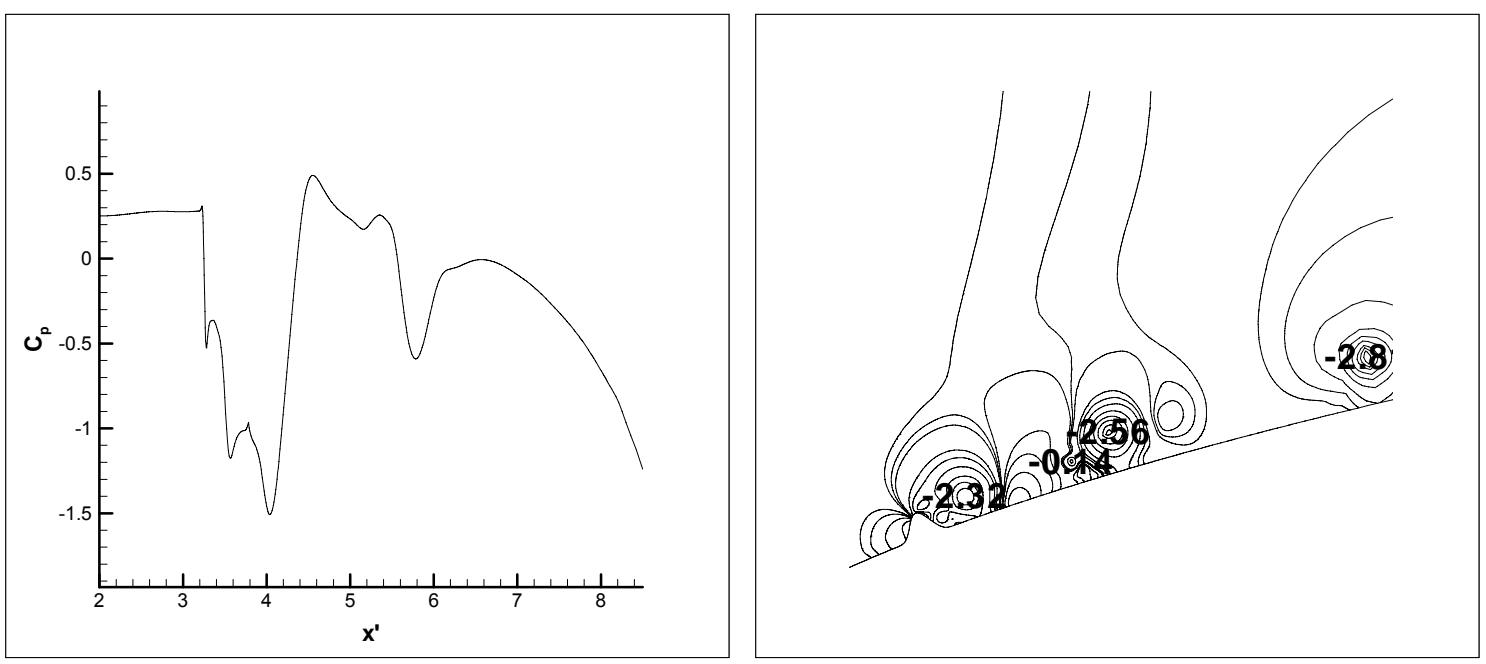

Figure 19. A snapshot from the unsteady flow: surface pressure and the pressure contours around a simulated roughness element. $R e_{l e r}=5000$.

\section{Effect of Ambient Relative Humidity}

The effect of ambient relative humidity can be seen by simple mathematical analysis as shown below for a constant mixing ratio assumption.

$$
R H=\frac{R H}{R H_{\infty}} R H_{\infty}=\frac{e / e^{*}}{e_{\infty} / e_{\infty}^{*}} R H_{\infty}=\frac{e / e_{\infty}}{e^{*} / e_{\infty}^{*}} R H_{\infty}=\frac{f(p)}{f(T)} R H_{\infty}
$$

Once the flow field is computed, the first term in the upper expression, $\frac{f(p)}{f(T)}$, becomes fixed. Than, as the relative humidity in the approaching flow is changed, so will the relative humidity levels in the flow field with a linear behavior.

In order to analyze the effect of the ambient relative humidity on the relative humidity variation over the region of interest, the same converged flow solution over the parabola at zero-degree angle-of-attack with a single hump is assumed as the base solution (cases with $R e_{l e r}=5000$ and $R e_{l e r}=10000$ ). Several relative humidity values with respect to liquid water are used $(80,90,100 \%)$ for the upstream flow, and for each case, the relative humidity value in the vortex core is recorded right after it detaches from the hump surface.

These records of the local relative humidity are plotted against the relative humidity in the approaching flow at different Reynolds numbers. Figure 20 -a shows the variation of the core relative 
a)

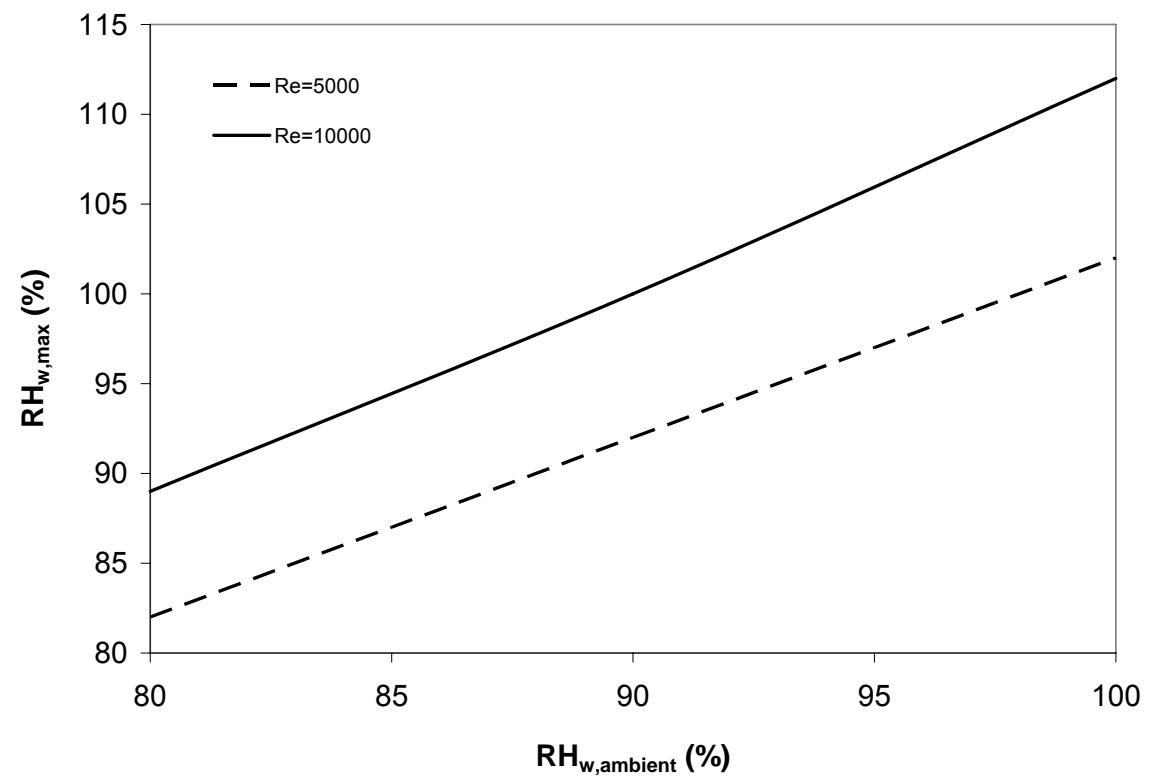

b)

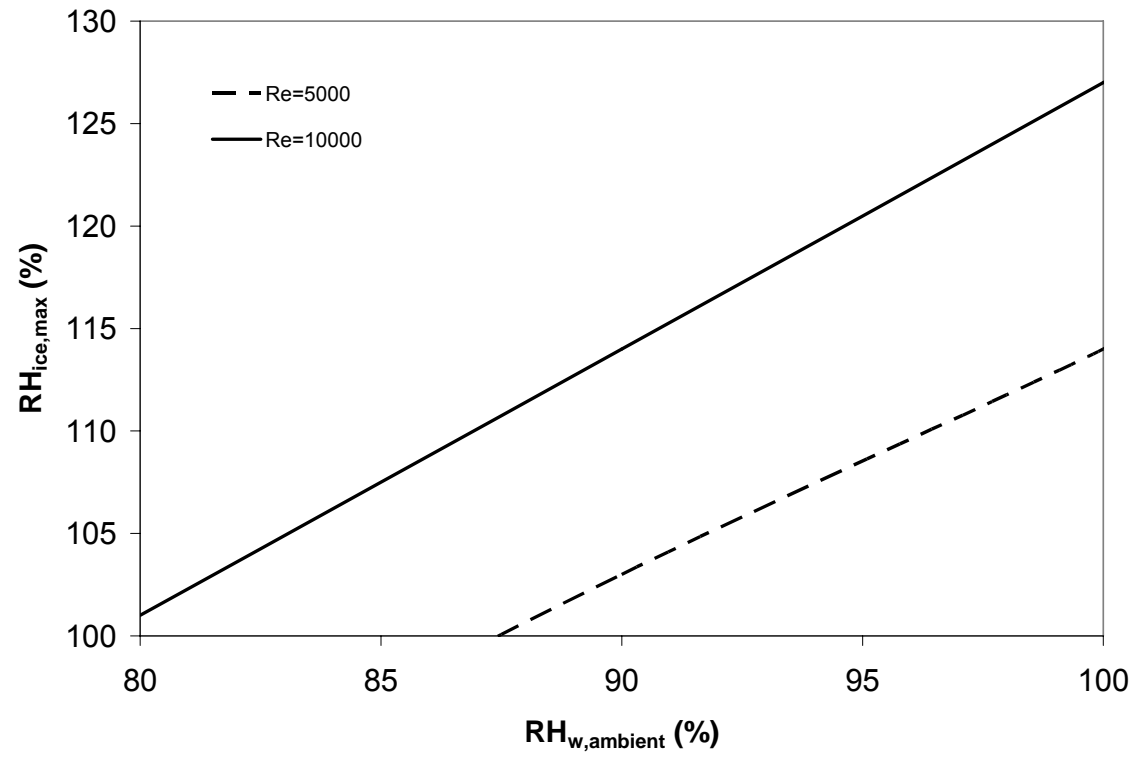

Figure 20. Variation of maximum $R H_{w}$ and $R H_{\text {ice }}$ due to the ambient relative humidity.

humidity with respect to water and Figure 20-b shows the variation of the core relative humidity with respect to ice. There is a linear relation between the ambient relative humidity and vortex-core relative humidity for both cases $\left(R H_{w}\right.$ and $\left.R H_{i c e}\right)$. However, the relative humidity values with respect to ice are much higher compared to the values of the relative humidity with respect to liquid water. By comparing Figure 20-a and Figure 20-b, one can see conditions where the free stream is subsaturated but locally becomes supersaturated with respect to both liquid water and ice. Also it is possible to see that locally, the 
flow is subsaturated with respect to liquid water but is supersaturated with respect to ice. These effects become more pronounced as the Reynolds number is increased. For example, considering Figure 20-a, for a $95 \% R H_{w}$ in the ambient flow, the vortex at $R e_{l e r}=5000$ has a subsaturated core whereas the vortex core at $R e_{l e r}=10000$ is supersaturated.

By comparing the lines for $R e_{l e r}=5000$ and 10000 , it is seen that the pressure at the core of the shed vortex drops as the Reynolds number is increased, thus increasing the local supersaturation level. In terms of Figure 20, a $6 \%$ increase in $R H_{w}$ and an $8 \%$ increase in $R H_{\text {ice }}$ is observed for a Reynolds number increase from $3.15 \times 10^{5}$ to $6.3 \times 10^{5}$ based on the chord length. This gives a clue of the importance of potential relative humidity effects for the practical cases where flight Reynolds numbers are on the order of millions. Therefore, it can be concluded that the size of the roughness element and the Reynolds number directly impact the potential for condensation and deposition due to supersaturation.

\section{Effect of Angle-of-Attack}

Multiple runs were done varying only the angle-of-attack with $\operatorname{Re}_{l e r}=100\left(R e_{c} \cong 6300\right)$. The main behavior of surface pressure over the analytic hump is first a slight increase due to slow down at the onset of the hump, then an abrupt decrease of pressure due to the acceleration of the flow over the hump, followed by a pressure plateau due to the separation bubble, and finally recovery of the parabola surface pressure. Qualitatively, this behavior of the surface pressure was observed to be the same no matter what angle of attack is used.

The surface pressure comparison for the runs made at different angles-of-attack is shown in Figure 21. From this figure, it is seen that even the magnitude of the relative drop in pressure caused by the roughness is the same for 0 to 6 degrees angle of attack. However, after 6 degrees, this effect begins to decrease. Due to the increase in the angle of attack, the leading-edge region of the airfoil starts to become the dominant obstacle for the flow and reduces the perturbation effect of the hump. This explains the relative reduction of the effect of the hump at higher angles of attack. Similarly, its favorable effects for the supersaturation are also suppressed as the angle-of-attack approaches the stall angle.

\section{Flow Field over Complex Ice Roughness}

After analyzing the impact of a single roughness element, an experimental ice shape along with the corresponding airfoil were input to the flow solver. It was observed that the presence of the complex ice roughness caused an unsteady flow where multiple vortices were formed and shed from the rough surface. This is particularly true at an angle of attack; the upper side of the ice is more susceptible to vortex 
shedding. The chord Reynolds number for the case that is considered in this study is approximately $8 \times 10^{5}$ for a chord length of 21 inches. The corresponding pressure field at a snapshot during the unsteady vortex shedding is given in Figure 22. The whole region above the upper ice surface is mainly under low pressure. The vortex cores are the peak points where the pressure finds its minimum values. $C_{p}$ values as low as -2.26 are obtained within the vortex shedding. One thing to note about these pressure values is that due to the conservative character of the pressure solver, the presented relative humidity values are conservative, as well.

The corresponding relative humidity values with respect to liquid water are given in Figure 23. Much of the upper and lower surface area is in a supersaturation state, with its peak values as high as $18 \%$ at the vortex cores. As expected, the stagnation region is in a subsaturation state due to increase in pressure and temperature. The relative humidity variation with respect to ice for the same flow is shown in Figure 24. Supersaturation with respect to ice is higher than that with respect to liquid water; this explains the supersaturation with respect to ice at the stagnation region. The overall leading-edge area is in an ice supersaturation state and a peak value of 35 percent is observed at the vortex cores. This demonstrates the potential for the deposition in these areas.

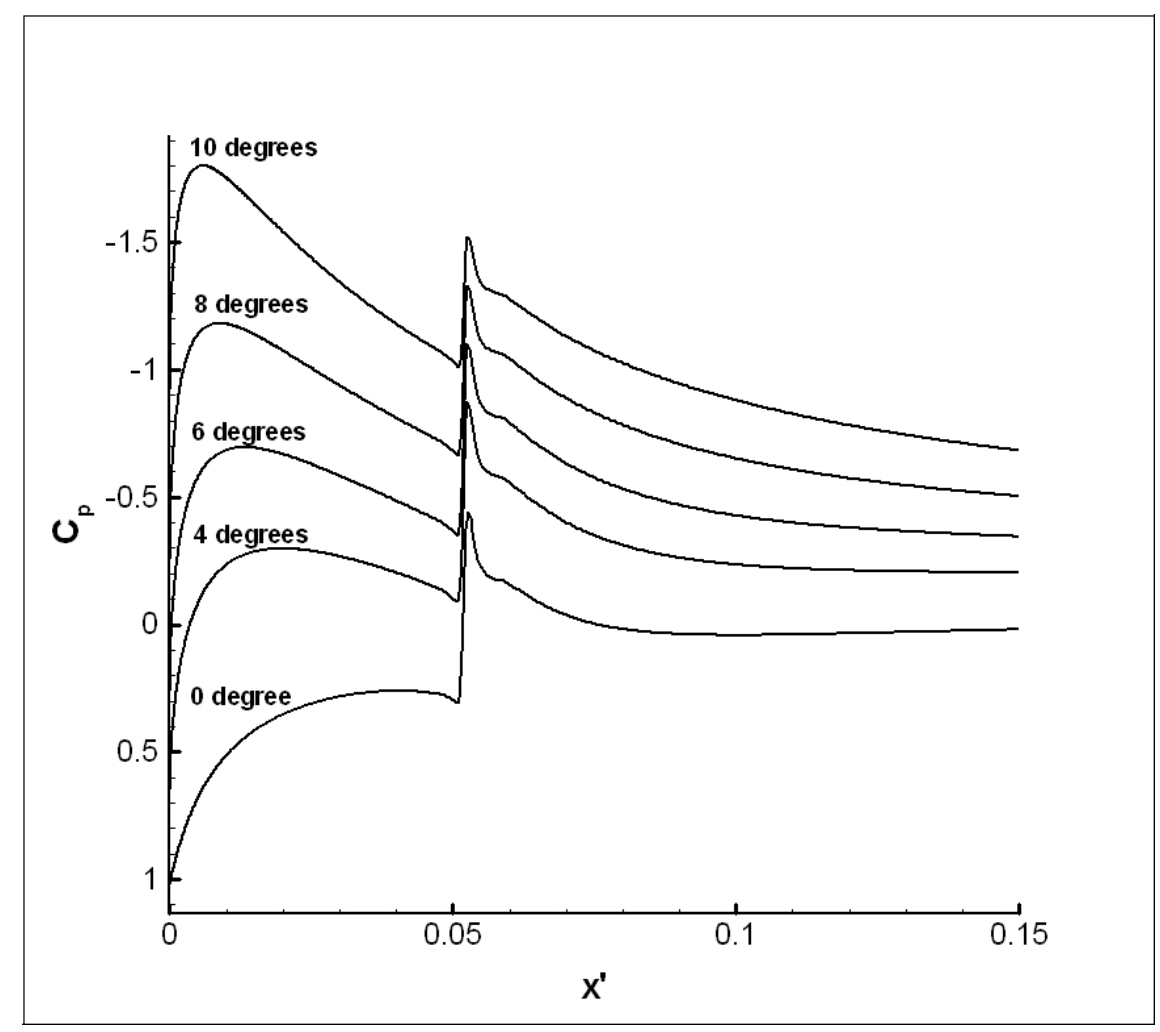

Figure 21. $C_{p}$ variation over an analytic hump on upper surface at different angles of attack. $R e_{l e r}=100$. 


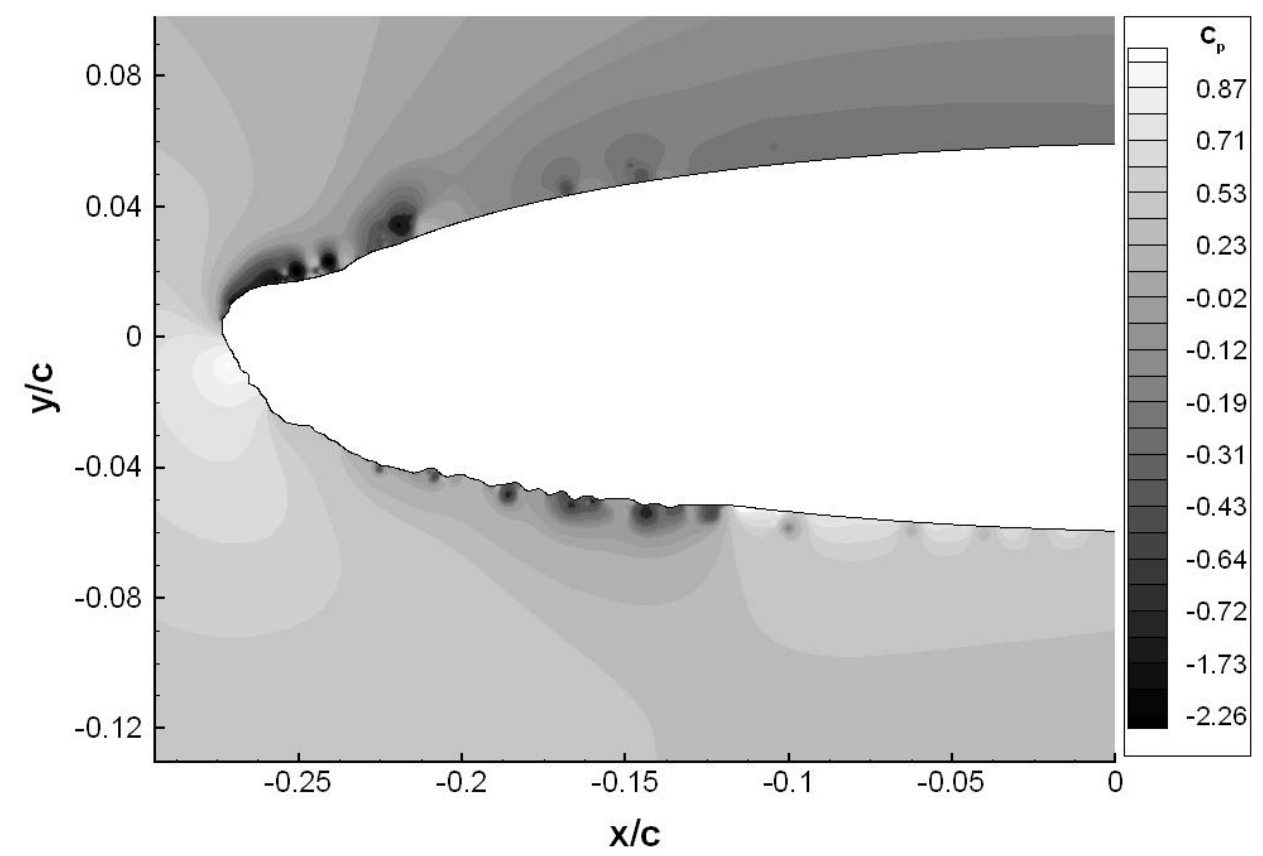

Figure 22. $C_{p}$ contours around ice accreted on NACA 0012 at $1^{\circ}$ angle-of-attack.

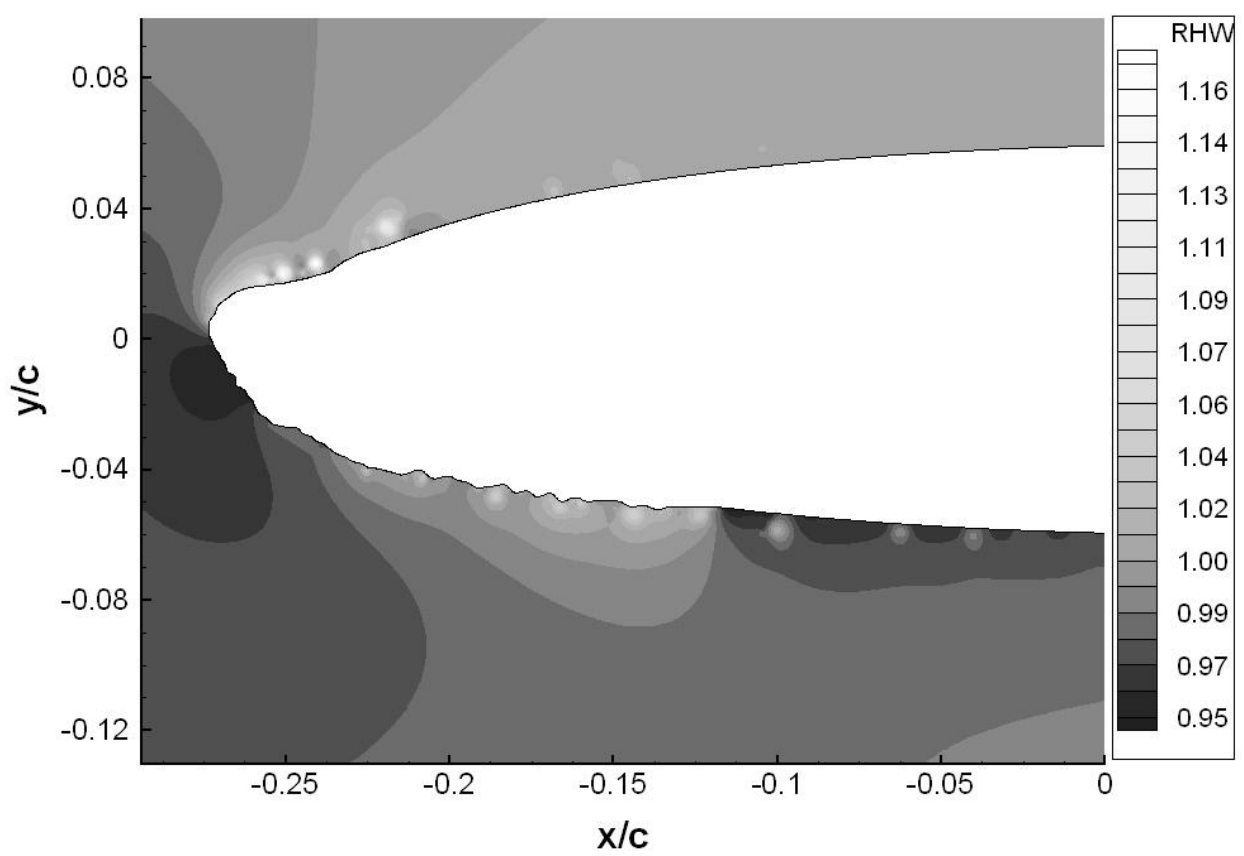

Figure 23. $R H_{w}$ around ice accreted on NACA 0012 at $1^{\circ}$ angle-of-attack. 


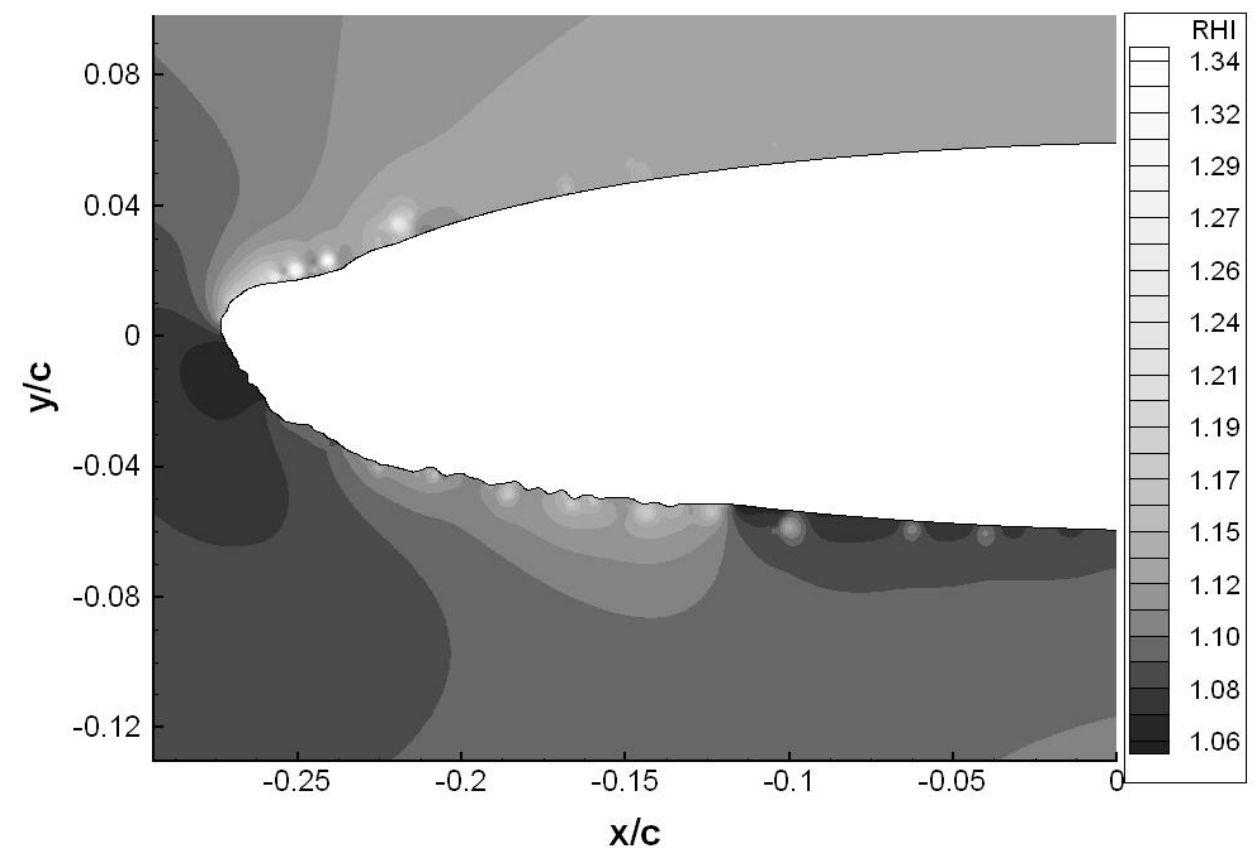

Figure $24 . R H_{i c e}$ around ice accreted on NACA 0012 at $1^{\circ}$ angle-of-attack.

Typically in the atmosphere, it is difficult to achieve supersaturation with respect to liquid water of more than $1 \%$ because the vapor begins to condense and always tries to return to an equilibrium state ${ }^{30}$. However, supersaturation with respect to ice can reach higher values for it is a much slower process. In this study, the potential of the relative humidity effects is being investigated. So, the high supersaturation values with respect to liquid water seen in these figures (23 and 24) can be thought of as a potential for condensation. Considering the fact that fresh air, which is saturated with water vapor, is constantly being fed into this supersaturated region, small droplets of water or ice are very likely to grow as they convect through the low-pressure regions and contribute to the formation of downstream frost/ice.

In addition to the shed vortices from the ice surface, there are also other vortices which are entrapped within the ice roughness. Given the complex roughness geometry, vortices can form within the small cavities of the ice roughness. These vortices, just like the ones shed from the upper surface, induce lowpressure regions, which in turn produce a strong potential for condensation/deposition with the excess vapor in the air. Considering the fact that the velocity is usually lower in a recirculating region, there is also more residence time for condensation and deposition to occur in these regions.

Figure 25 shows the variation of the vortex core relative humidity as a function of the ambient relative humidity. The ambient relative humidity is varied from $80 \%$ to $110 \%$ with respect to liquid 
water. The relative humidity values in the core of the vortex are plotted both with respect to liquid water and with respect to ice. The vortex core becomes supersaturated with respect to liquid water only when the ambient relative humidity level exceeds $91 \%$, whereas it is supersaturated with respect to ice for all of the values in the figure. If there is no allowance for a phase change due to condensation or deposition, then for relative humidity values greater than $91 \%$ in the approaching flow, the vortex core becomes heavily supersaturated. This result again reinforces the conclusion that vortices which are formed due to the ice roughness on the surface are effective mechanisms for ice or water droplet growth.

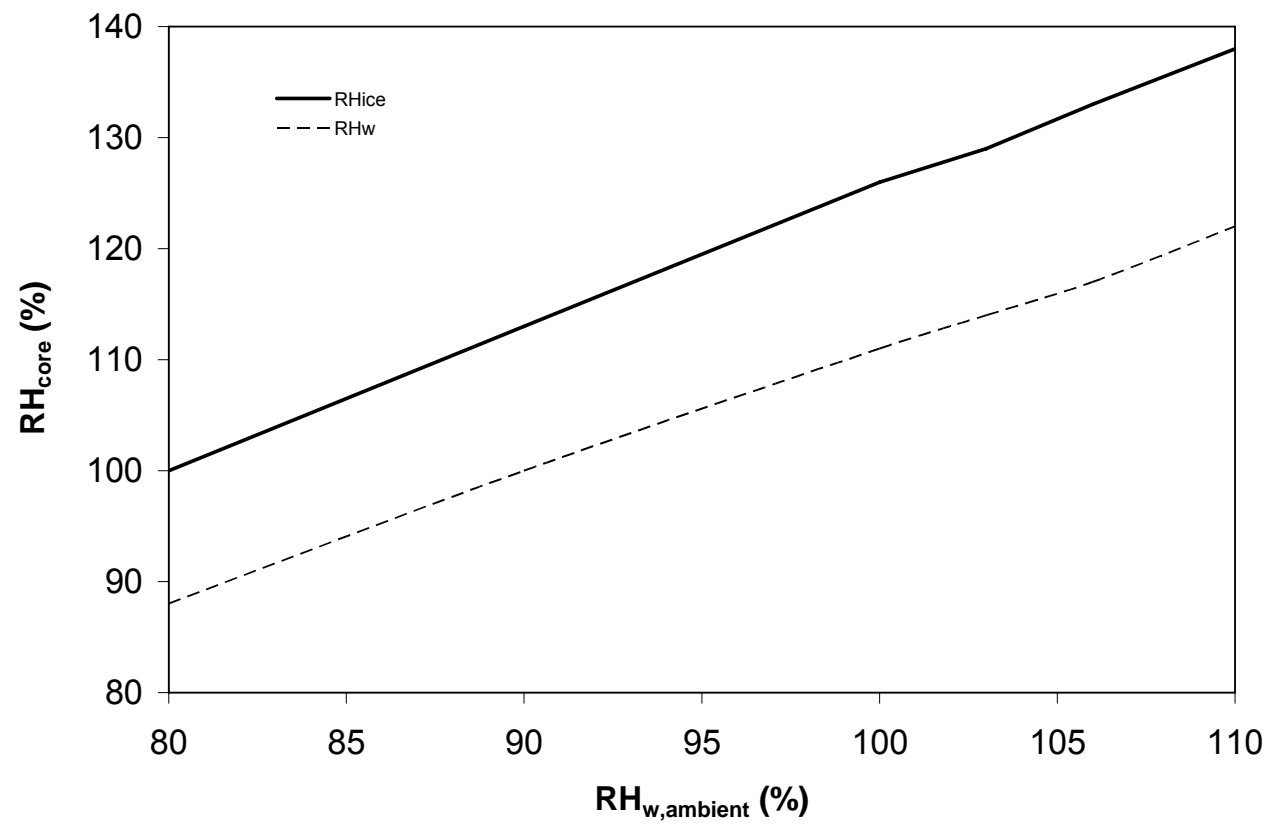

Figure 25. Variation of the vortex core relative humidity due to the ambient relative humidity.

\section{DISCUSSION}

It should be noted that the $2 \mathrm{D}$ nature of the calculations restricts the flow so that the variations in pressure are more pronounced compared to a 3D case with the same geometry. The impact of this on the saturation levels is that the calculated level of local supersaturation will be higher compared to a 3D case. On the other hand, the conservative nature of the pressure solver leads to less drop in pressure. Therefore, for a more accurate computation, 3D modeling of the flow over the entire wing is essential.

One of the assumptions in this part of the study is that any kind of phase change from the vapor phase has been neglected. However, in a real life application there are liquid water droplets in the air; and as the flow approaches the stagnation point, the temperature increases due to aerodynamic compression. This 
heating can cause droplet evaporation. The effect of this heating is more significant on the smaller droplets which may form as a result of a splash. This mechanism causes the droplets to evaporate and increases the local water vapor level. This extra vapor content will then be condensed as the flow passes through the suction areas. However, this effect has been excluded in the present study. Also, ventilation due to cross-flow, turbulence and compressibility effects are known to affect the vapor transport, hence the local relative humidity, which are all excluded in the present study. Therefore, for a complete analysis, the phase changes, ventilation, turbulence, compressibility effects, and the 3D structure of the flow must be considered. 


\section{CHAPTER IV}

\section{MECHANISMS FOR DOWNSTREAM ICE GROWTH}

This study has been presented at the

SAE World Aerospace Congress, October 2005

2005-01-3375

\section{INTRODUCTION}

Numerical simulation of aircraft icing has various applications such as aircraft certification, flight simulator development and wind tunnel test result prediction ${ }^{11}$. The resulting ice shape in a numerical model heavily depends on the collection efficiency distribution over the exposed airframe surface. This distribution varies by time due to the interaction between the changing surface geometry and the flow field. Because of this complexity, calculation of the collection efficiency $(\beta)$ is one of the most difficult steps of ice accretion prediction and has vital importance for the accuracy and reliability of the numerical predictions.

The calculation of the collection efficiency involves the tracing of the droplets in the flow field. There are two different approaches for this purpose ${ }^{2}$ : Eulerian and Lagrangian. In the case of a Lagrangian approach, which is widely used in the aerospace community, the individual droplets are tracked in the flow field subject to different forces such as drag, gravity, and buoyancy. For the Eulerian approach, the droplets are considered as a continuum where the droplet density reflects the number density of the droplets. Each of these approaches has its own advantages and disadvantages. Typically, for a 2D computation, it is better to assume a Lagrangian approach, where as a $3 \mathrm{D}$ computation may require an Eulerian approach in order to decrease the computational load and cost ${ }^{31}$.

The droplets that are used in numerical studies usually have less than $40 \mu \mathrm{m}$ MVD. These droplets are expected to follow the streamlines reasonably well. The inertia of these droplets, however, causes them to impinge on the wing surface and form ice. In order to compute the droplet trajectory, the following assumptions are generally used ${ }^{32}$ :

- Flow field and the droplets are de-coupled, so that the droplets do not alter the flow,

- The droplet radius remains constant until it hits the surface, hence no change in droplet water content by any means,

- The splashing effects are negligible so that there is not a re-distribution of water after splash, and

- The droplets are assumed to have a spherical shape for drag computation purposes. 
The extended research and in-flight observations have shown that, especially in mixed-phase clouds, droplets of much larger size can be encountered ${ }^{32}$. The MVD for these droplets can be on the order of hundred microns. These droplets do not comply with the above mentioned assumptions and cause unpredictable ice shapes due to different underlying mechanisms. Some of these mechanisms are:

Droplet shape distortion leading to a different drag force, hence different path, than expected ${ }^{33}$,

- Droplet break-up before impingement ${ }^{33}$,

- Splashing, re-impingement and consequent water redistribution ${ }^{34}$,

- Water film dynamics ${ }^{12}$,

- Impact on heat transfer characteristics, and

- Ice sublimation ${ }^{35}$.

An example feature of the resultant ice shapes due to these extra mechanisms is the feather growth, which takes place beyond the calculated impingement region ${ }^{35}$. As the feathers grow, not only do they distort the oncoming and downstream flow, but they also constitute an added impingement region which protrudes into the flow. Therefore, droplets that by-pass the primary leading-edge region can be important due to their contribution to ice growth downstream ${ }^{35}$. This shows clear evidence of the importance of accurate droplet trajectory calculations.

Another mechanism for downstream ice growth is the local variations in relative humidity. Currently, the effects of the relative humidity are neglected in ice shape prediction considering that "it is at best a secondary effect" ${ }^{\prime 7}$. However a previous study ${ }^{28}$ showed that there is a potential for the relative humidity to impact the ice growth due to local supersaturation. It was reported that depending on the time scales and the level of subsaturation and/or supersaturation, the liquid water distribution on the wing surface can be affected.

The present work is a preliminary study on relative humidity effects and vortex interaction as mechanisms for downstream ice growth (i.e. ice formation beyond the primary leading-edge ice shape). The local relative humidity levels and the associated time scales were investigated. The effect of the local flow dynamics on the droplet path was also investigated. The assumptions that are used in the Lagrangian particle tracer are the same as the ones used by $\operatorname{Tan}^{3}$, but the relative humidity effects are left optional in order to investigate the impact on the droplet path.

The second part of the work is a study of time scales involved in the processes triggered by saturation effects. These processes are expected to impact the local liquid water content $(L W C)$ and vapor water content $(V W C)$ values. In order to perform this study, an air volume of $1 \mathrm{~m}^{3}$ is assumed and the number of droplets in this volume is calculated based on the median volumetric diameter $(M V D)$ and $L W C$ values. 
The droplets are assumed at the same initial radius, which is half of the $M V D$. The system is assumed to be in equilibrium in terms of vapor-liquid phases with an initial relative humidity of $100 \%$ with respect to liquid water. This volume then undergoes a sudden pressure change, which results in a sudden change in the relative humidity. As the system returns to vapor-liquid equilibrium where the relative humidity is again $100 \%$, the variation of the droplet radius is traced as a function of time. The assumptions for this part of the study are as follows:

- Temperature calculated from the isentropic relation,

- Latent heating due to condensation is included,

- Only saturation with respect to liquid water is considered,

- Phase change between the vapor phase and the liquid phase is investigated,

- Curvature effects for the droplets are neglected

- Water vapor and liquid water droplets are uniformly distributed in the control volume at all times.

\section{GOVERNING EQUATIONS}

A two-dimensional Navier-Stokes algorithm, capable of capturing the unsteady vortex shedding process, was used for the flow simulations. Thorough validation and documentation of this code is available in Refs. [14, 19 and 28]. Local relative humidity variations were calculated by employing a pressure solver along with a subroutine that calculates the relative humidity field with respect to liquid water. The details have been covered in Chapter III, so will not be repeated here for brevity. However, a separate analysis was made in this study to determine the time scales that are required to see a significant impact due to relative humidity.

\section{Model for Time Scales: Box Experiment}

In this part of the study, a control volume of unit size was selected at $100 \%$ initial relative humidity with respect to liquid water. The initial LWC and MVD were also specified. These two parameters have been used to determine an average number of droplets in the control volume using the following formula:

$$
N=\frac{\left(L W C / \rho_{\text {water }}\right)}{\frac{4}{3} \pi\left(\frac{M V D}{2}\right)^{3}}
$$

where $N$ is the number density of the droplets. In this formula, the numerator gives the total volume of the liquid water in the unit volume, and the denominator gives the average volume of a single droplet. The number density is used to account for the total changes within the volume in terms of $L W C$ or $V W C$. Other input parameters are the static pressure and air density to simulate the desired altitude. 
The numerical experiment that was performed includes the following steps. A sudden temperature change is imposed isentropically on the control volume, which also changes the pressure. The change in pressure alters the saturation level of the system, which was set to be $100 \%$ (saturated) initially. What is expected is that due to the condensation/evaporation of the droplets within the volume, the system is going to come to a new equilibrium condition where relative humidity is again $100 \%$ with respect to liquid water at the imposed pressure level. During this process, the droplet radius, $L W C$, and $V W C$ are recorded as a function of time until a specified convergence criterion is met.

In order to calculate the value of the pressure that is impulsively imposed on the control volume, a flight speed and altitude must be specified. Then for a given pressure coefficient $\left(C_{p}\right)$, one can calculate the real value of the corresponding static pressure. New thermodynamic values for this imposed state are calculated using the isentropic relation. As the system restores equilibrium, its pressure is going to stay constant. However, the density and temperature, hence the saturation vapor pressure, $e^{*}$, are going to vary due to latent heating.

For this numerical experiment, the following methodology was used ${ }^{28}$ :

1. Specify a time step for integration (e.g. 0.1 second).

2. For the given initial temperature (before the impulsive start), get the saturation vapor pressure, $e^{*}$.

The air is at $100 \%$ relative humidity with respect to liquid water initially. So, $e=e^{*}$, which is the initial vapor pressure.

$$
e=e_{\text {initial }}^{*}=6.112 \exp \left(\frac{17.67 T}{T+243.5}\right)
$$

where $e^{*}$ is in millibars and temperature in Celsius.

3. Calculate the imposed temperature using the ideal gas relation.

$$
\left(\frac{T}{T_{\text {initial }}}\right)=\left(\frac{P}{P_{\text {initial }}}\right)^{\frac{\gamma-1}{\gamma}}
$$

4. Calculate the saturation vapor pressure, $e^{*}{ }_{n e w}$, for new conditions.

5. Update the new relative humidity value by

$$
R H_{w}=e / e^{*}
$$

6. Update the radii $(r)$ of the droplets using the equation below. 


$$
\frac{d r}{d t}=\frac{c}{r}, \text { where } c=\frac{R H_{w}-1}{\left[\left(\frac{L}{R_{v} T}-1\right) \frac{L \rho_{\text {water }}}{K T}+\frac{\rho_{\text {water }} R_{v} T}{D e^{*}}\right]}
$$

7. To find the change in the $L W C$, multiply the volume change of a droplet by the density of water and by the number of droplets in the control volume.

$$
\Delta L W C=\Delta V_{\text {droplet }} \rho_{\text {water }} N=\frac{4}{3} \pi\left(r^{3}-r_{o}^{3}\right) \rho_{\text {water }} N
$$

8. Then update the $L W C$ and the $V W C$

$$
L W C_{n e w}=L W C+\Delta L W C \quad V W C_{n e w}=V W C-\Delta L W C
$$

9. Update the temperature using the latent heat release:

$$
\Delta T=\frac{L \Delta L W C}{\left(m C_{p}\right)_{a i r}}
$$

10. Update the volume using the ideal gas equation:

$$
\Delta V=\frac{m_{\text {air }} R_{\text {air }}}{p} \Delta T
$$

11. $V W C_{\text {new }}=\rho_{v}$, so using this vapor density, calculate the new value of the vapor pressure, $e$.

$$
e=\frac{w p}{0.622+w}, \text { where } w=\rho_{v} / \rho_{\text {air }}
$$

12. Continue the process by marching in time by following the steps 4-11 until the $L W C$ and $V W C$ do not change significantly and a new equilibrium is reached.

\section{Particle Tracing}

The Lagrangian particle tracing in this study is a post process that inputs a flow field solution from the 2D-NS flow solver ${ }^{14,19}$. The advantage of this approach is that the effects of the local flow dynamics, such as steady separation bubble or unsteady vortex shedding, are revealed and the subsequent impact on droplet collection efficiency $(\beta)$ can be investigated. The forces acting on a particle were calculated according to the subsections below:

\section{$\underline{\text { Drag Force }}$}

The drag coefficient acting on the spherical droplet is calculated by the following formula ${ }^{36}$ :

$$
C_{d_{\text {sphere }}}=\frac{24}{\operatorname{Re}}+\frac{6}{1+\sqrt{\operatorname{Re}}}+0.4 \text { valid for } 0 \leq \operatorname{Re} \leq 2 \times 10^{5}, \text { where } \operatorname{Re}=\frac{2 U r}{v_{\text {air }}}
$$


In the definition of the Reynolds number, the speed of the flow is relative to the sphere. In this case, both the particle and the flow have speeds as a function of time and space. Therefore, the relative speed of the wind with respect to the sphere needs to be calculated:

$$
U=|\vec{U}|=\left|\vec{U}_{\text {local flow }}-\vec{U}_{\text {droplet }}\right|
$$

Based on this speed, the Reynolds number and the corresponding drag coefficient can be calculated. The drag force acting on the sphere was calculated from the standard relation:

$$
\operatorname{Drag}_{\text {sphere }}=\frac{1}{2} \rho_{\text {air }} U^{2} S C_{d_{\text {sphere }}} \text {, where } S=\pi r^{2}
$$

Gravity and Buoyancy

Gravitational force exhibits itself in the negative y direction and is constant in the computational field. Due to the incompressible character of the flow solver, the buoyancy force is also constant in the computational field. The following formula is used for the force due to gravity and buoyancy, which are purely in $y$-direction:

$$
F_{\text {gravity and buoyancy }}=-m_{\text {droplet }} \frac{g\left(\rho_{\text {water }}-\rho_{\text {air }}\right)}{\rho_{\text {water }}}
$$

Calculation of the Accelerations

Calculation of the mass is the product of the sphere volume and the water density.

$$
\text { mass }=\frac{4}{3} \pi r^{3} \rho_{\text {water }}
$$

The acceleration has the units of velocity divided by time. The velocity is non-dimensionalized by the far upstream flow speed $\left(V_{\infty}\right)$, and the time is non-dimensionalized by using the ratio of the leading-edge radius $(l e r)$ and $V_{\infty}$. Therefore, physical acceleration is non-dimensionalized with the term:

$$
a^{*}=\frac{V^{*}}{t^{*}}=\frac{V_{\infty}}{\left(l e r / V_{\infty}\right)}=\frac{V_{\infty}^{2}}{l e r}
$$

Numerical Approach for the Local Values

The calculation of the velocity difference requires an interpolation to determine the local wind speed, since the particle can be located at a point within the grid cell. This interpolation assumes a rectangular cell and determines the values as follows: 


$$
\begin{aligned}
u_{\text {local }}= & u_{\text {lower left }}+\frac{\partial u}{\partial \xi} \Delta \xi+\frac{\partial u}{\partial \eta} \Delta \eta= \\
& u_{\text {lower left }}+\frac{u_{\text {lower right }}-u_{\text {lower left }}}{\xi_{\text {lower right }}-\xi_{\text {lower left }}}\left(\xi_{\text {droplet }}-\xi_{\text {lower left }}\right)+ \\
& \frac{u_{\text {upper left }}-u_{\text {lower left }}}{\eta_{\text {upper left }}-\eta_{\text {lower left }}}\left(\eta_{\text {droplet }}-\eta_{\text {lower left }}\right)
\end{aligned}
$$

where $\xi$ and $\eta$ are the coordinates in the transformed plane that were used in the flow field calculations. The $v_{\text {local }}$ was found in a similar fashion.

\section{Calculation of $L W C$ for the Simulated Unsteady Flow}

In order to calculate the potential for an increase in ice thickness due to additional droplet impingements, it is useful to go over the formula that is used for $L W C$ in the simulated unsteady flow. The two assumptions for the calculation of $L W C$ are uniform droplet distribution in the given volume and constant droplet diameter at all locations. The temporal frames that were used in the simulation of the unsteady vortex field were 32.2 microseconds apart from each other. For a continuous droplet tracing in the unsteady process, droplets must be released at integer multiples of this time gap. Therefore, the separation between two consecutive droplets, $s_{d}$, is given by:

$$
s_{d}=V_{\infty} \Delta t=V_{\infty}\left(32.2 \times 10^{-6} n\right)
$$

where $n$ is an integer prescribed by the researcher (see Table 1). The only criteria when selecting the value of $n$ is that the calculated $L W C$ must be in the range that is used in the icing tunnel experiments. This range is roughly between 0.3 and $1.6 \mathrm{~g} / \mathrm{m}^{3}$.

Table 1. The number of frames used to determine the separation between the neighboring droplets and the corresponding $L W C$.

\begin{tabular}{|c|c|c|}
\hline $\begin{array}{c}\text { MVD } \\
\text { (micron) }\end{array}$ & $n$ & $\begin{array}{c}\text { LWC } \\
(\mathrm{g} / \mathrm{m} 3)\end{array}$ \\
\hline 20 & 3 & 0.50 \\
\hline 30 & 4 & 0.71 \\
\hline 40 & 6 & 0.50 \\
\hline 50 & 7 & 0.62 \\
\hline
\end{tabular}

If there is a uniform droplet distribution, then this separation must be the same in all three dimensions of space, which forms a cube of volume $s_{d}^{3}$. This means that one droplet is contained in every $s_{d}^{3}$ amount of volume. Then the total number of droplets in a unit volume is given by: 


$$
N=\frac{1}{s_{d}^{3}}
$$

The amount of liquid water in a unit volume of air, which is $L W C$, can be calculated by multiplying the number of droplets with the mass of a single droplet, as shown in Equation 63. The calculated values of LWC for the cases considered in the present study are given in Table 1.

$$
L W C=N m_{\text {droplet }}=N\left(\frac{4}{3} \pi\left(\frac{M V D}{2}\right)^{3} \rho_{\text {water }}\right)
$$

\section{RESULTS}

The results in this study include two parts, each addressing a different aspect of downstream ice growth.

\section{Relative Humidity}

In terms of the relative humidity, there are two main issues: the severity of the saturation levels and the time scales involved in the relative humidity induced processes.

\section{$\underline{\text { Relative humidity field }}$}

For the investigation of the relative humidity field, the flow solver and the pressure solver were used as explained in References 14, 19 and 28. The relative humidity was then post-processed using the calculated pressure field. As an example, one rime ice case and one glaze ice case were chosen, both experimentally obtained on a NACA 0012 airfoil. The relative humidity variations were calculated with respect to both liquid water and ice.

The case with the rime ice was run at a chord Reynolds number of $0.82 \times 10^{6}$. The experimental rime ice from the NASA Glenn IRT along with the surrounding relative humidity fields at a snapshot of the unsteady vortex shedding process are shown in Figure 23 and Figure 24. The relative humidity levels within the vortex cores were as high as $118 \%$ with respect to liquid water (RHW), and $135 \%$ with respect to ice (RHI). These vortices also impose a low pressure region on the airfoil surface, which in turn increases the local supersaturation level. This produces a strong potential for the accumulation of additional liquid water or ice on the surface through condensation or deposition.

The relative humidity fields for the glaze ice ${ }^{13}$ case $\left(\operatorname{Re}_{c}=0.5 \times 10^{6}\right)$ are shown in Figure 26 . Due to the highly rough nature of the existing ice surface, the vortex shedding was much more violent and the variations in pressure and relative humidity were much more severe. The relative humidity levels within 


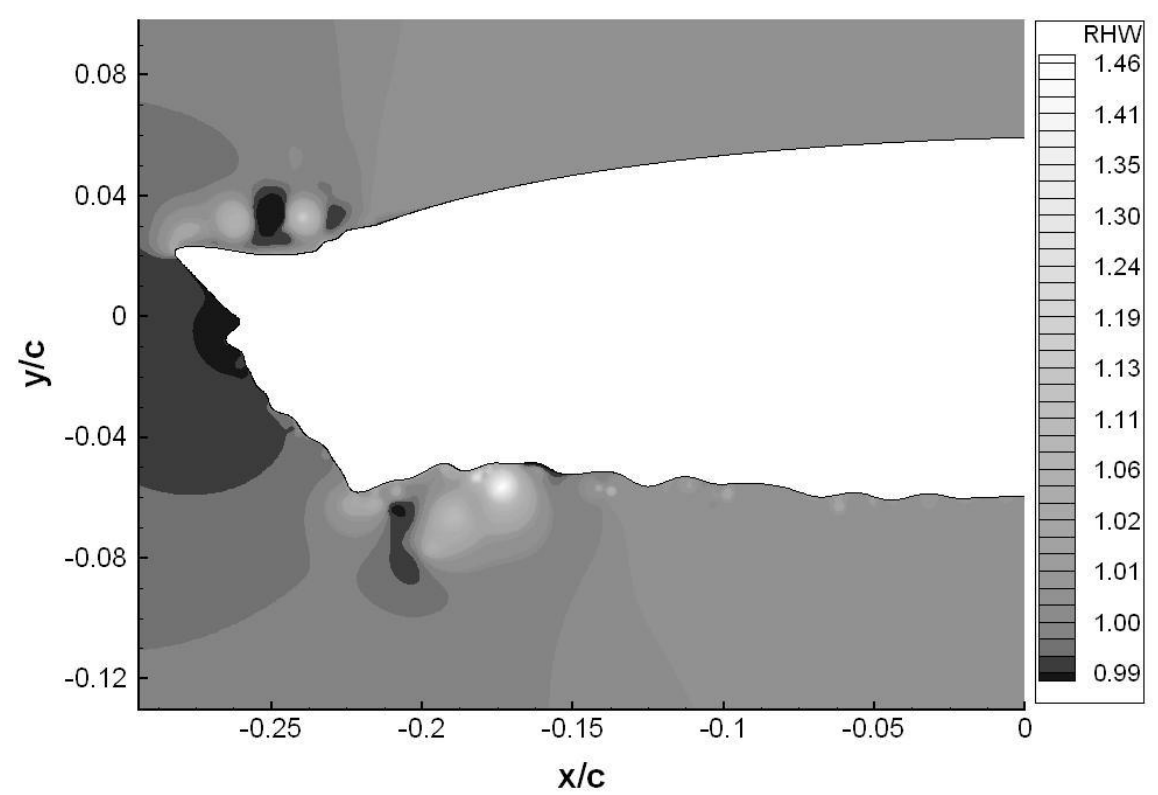

a) Relative humidity with respect to liquid water

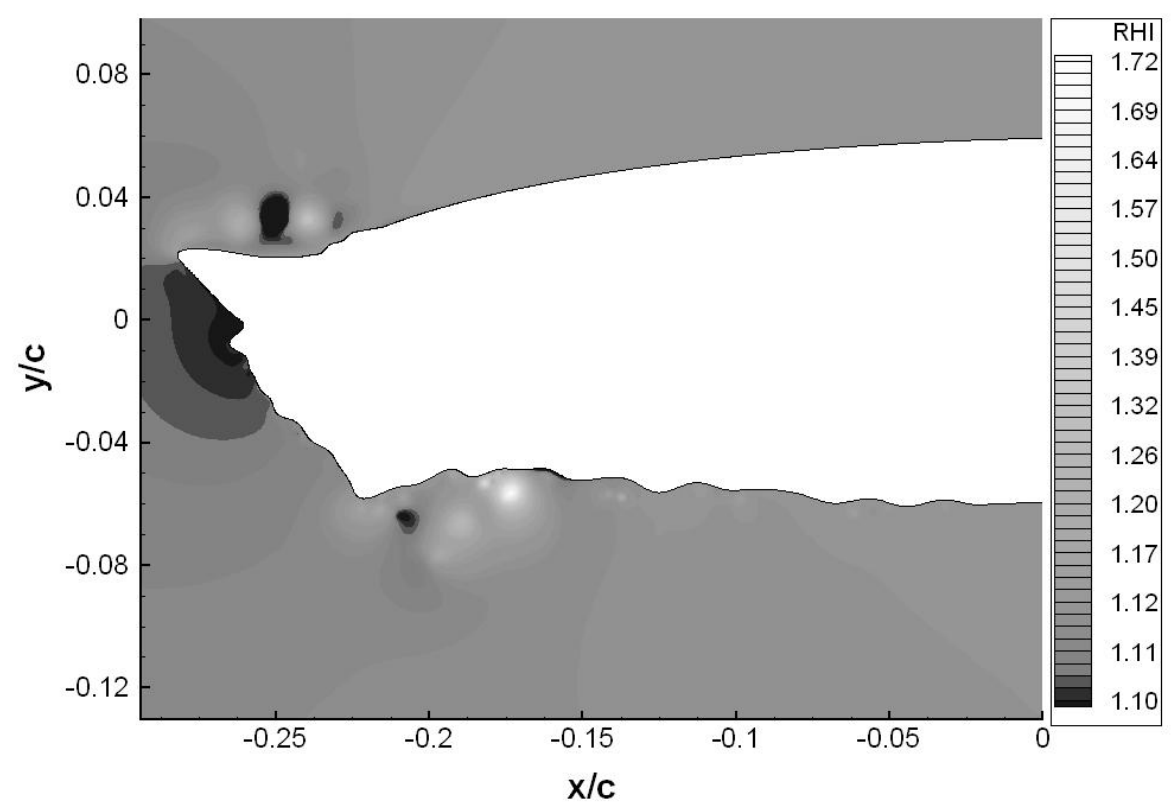

a) Relative humidity with respect to ice

Figure 26. Relative humidity field around NACA 0012 leading-edge with ice shape (AUG 1991 3-6$36)^{13}$. 
the vortex cores were as high as $147 \%$ with respect to liquid water (RHW) and $173 \%$ with respect to ice (RHI). The existence of these supersaturated regions is due, in part, to the roughness of the primary ice shape from direct droplet impingement. A prime example is the glaze horn present in Figure 26. So, once large ice perturbations such as this develop on the leading edge, aft regions can become quite susceptible to highly supersaturated air at or near the surface, which promotes additional accretion through condensation and deposition.

One important point to note in Figure 23, Figure 24, and Figure 26 is the extremely high values of supersaturation in the flow field. In the free atmosphere, the relative motion of the air is slow enough to allow any supersaturation to be cleared through either condensation or deposition. For the cases presented here, the water condensation and deposition due to supersaturation are avoided per the methods followed in the existing ice accretion codes. The reason for taking such an avenue is that the contribution from the local changes in relative humidity is neglected in the existing ice accretion codes. So, it is desired in this study that the consequences of such an assumption are shown. Another reason for these high numbers is that the fields shown in these figures represent a transient state due to the strong forcing effect of the iced wing surface at this high Reynolds number. The excess humidity does not have enough time to completely condense within the given small time scale. Once the air is past the wing surface, the transient state quickly disintegrates into normal states in the atmosphere.

\section{$\underline{\text { Available Water }}$}

In order to see the upper limit of relative humidity effects, a simple calculation was made in order to see the amount of excess water in case of supersaturation at different levels. The amount of water in $1 \mathrm{~m}^{3}$ of air is plotted against the relative humidity with respect to liquid water in Figure 27. Although not shown, as higher levels of supersaturation are achieved, this curve asymptotically approaches to 1.32 $\mathrm{g} / \mathrm{m}^{3}$, which is the total amount of water vapor in the $100 \%$ saturated air of unit volume at $5000 \mathrm{~m}$ altitude for standard atmosphere conditions.

The non-dimensional pressure $\left(C_{p}\right)$ distribution around an airfoil is almost constant for varying Reynolds number. However the actual pressure distribution indeed varies wit the Reynolds number. Higher Reynolds numbers cause higher drops in pressure, and therefore, they more abruptly achieve the high relative humidity values as compared to the lower Reynolds numbers. This phenomenon is depicted in Figure 28 for a $C_{p}$ value of -2 at $5000 \mathrm{~m}$ altitude in standard atmosphere. As the Reynolds number increases, the excess amount of water vapor in the air exponentially increases. Although not shown here, this curve eventually assumes an S-shape and asymptotically approaches to the same $1.32 \mathrm{~g} / \mathrm{m}^{3}$ value as higher Reynolds numbers are achieved for the same non-dimensional pressure. 


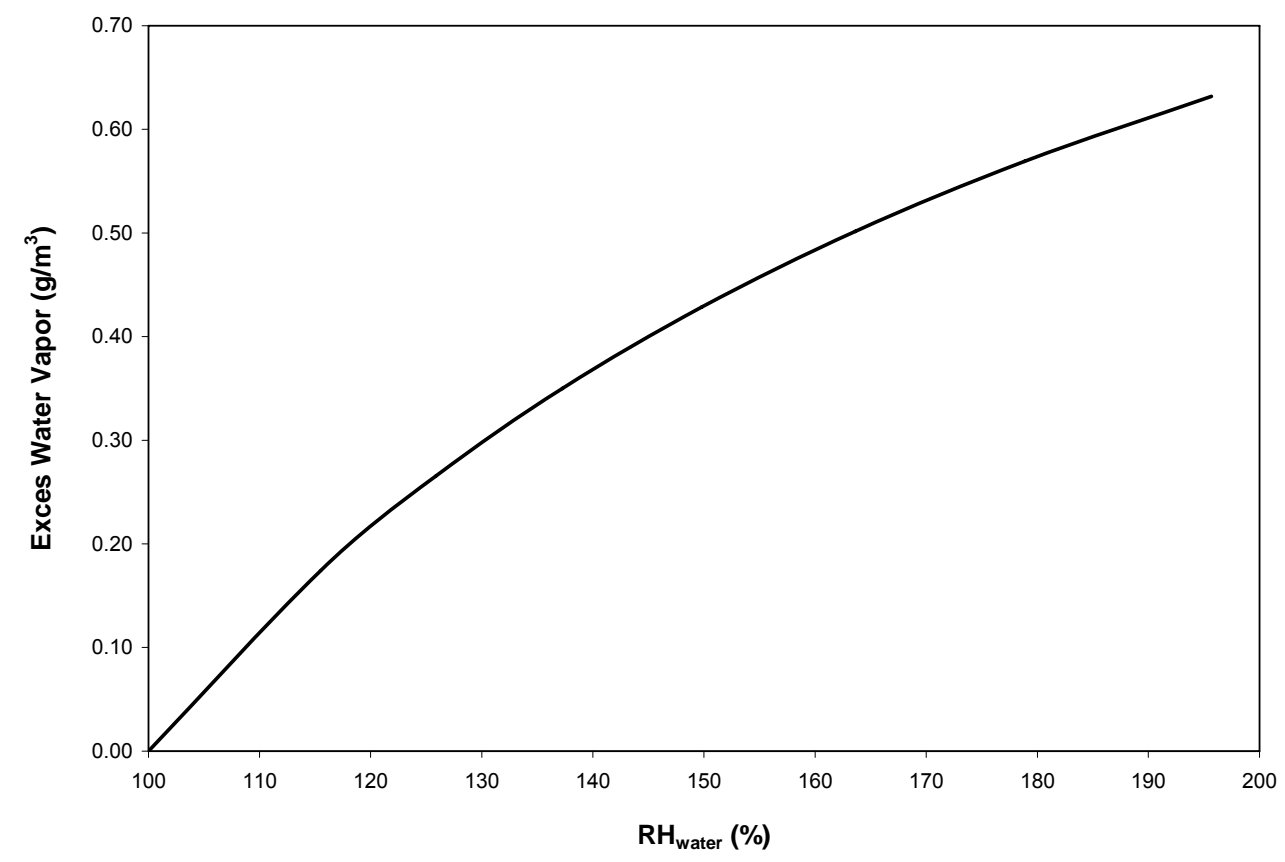

Figure 27. Variation of excess water vapor mass as a function of relative humidity with respect to liquid water; values are calculated for $5000 \mathrm{~m}$ altitude in standard atmosphere.

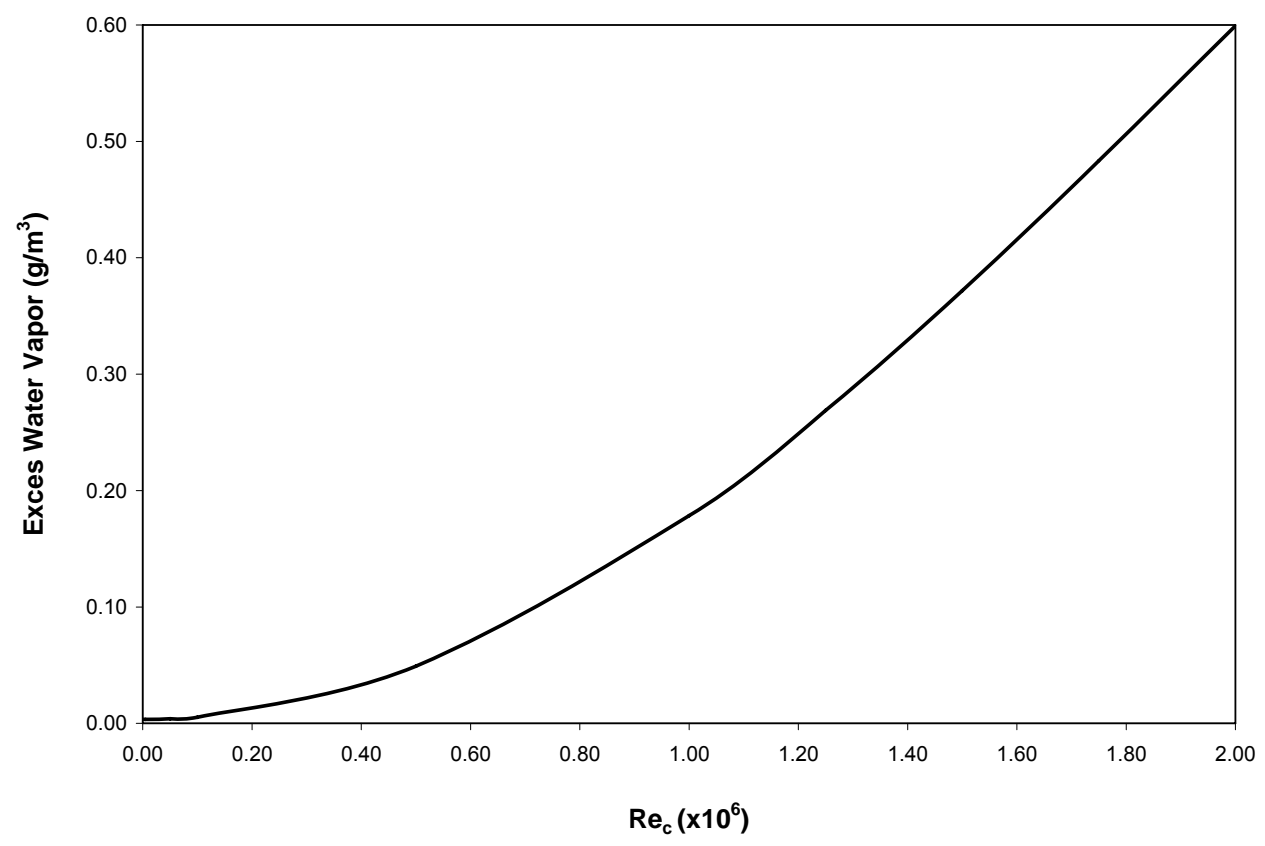

Figure 28. Variation of excess water vapor mass as a function of Reynolds number; values are calculated at $C_{p}=-2$ for a chord length of 21 inches at $5000 \mathrm{~m}$ altitude in standard altitude. 
For the icing experiments in wind tunnels, the $L W C$ values are usually between $0.3-1.6 \mathrm{~g} / \mathrm{m}^{3}$. The excess water vapor amount values shown in Figure 27 and Figure 28 are comparable to the amount of liquid water used in the experiments. This shows that, if the mechanisms involved in the ice accretion process are interacting with this excess water vapor, it can be expected that the resultant ice accretions are going to be different than the predicted ones, since relative humidity interactions are not considered in the current aircraft icing models.

\section{$\underline{\text { Time scales }}$}

The objective of this part of the study was to establish whether the residence time of the vapor-air mixture within the supersaturated regions was sufficient for any relative humidity induced effect to occur. The box experiment was performed for the investigation of the time scales in a stationary case. Figure 29 shows the $L W C$ history for different initial relative humidity levels with respect to liquid water. The initial diameter of the droplets was 10 microns for this case. As seen in this figure, the imposed level of relative humidity at the start of the experiment did not affect the time required to re-establish the equilibrium conditions. For all cases, the equilibrium recovery time was 1.6 seconds. However, it was seen that the initial relative humidity level determines the final $L W C$ at the equilibrium conditions, where relative humidity is again $100 \%$. Given sufficient time, the relative humidity has the potential to increase the $L W C$ in the approaching flow as much as $100 \%$, or decrease it $30 \%$. However, it was seen that the fime for this process can be too long in order to achieve completion over the leading-edge of a wing in an actual flight condition.

Further investigation of the time scales was done by keeping the initial relative humidity constant at $140 \%$ and varying the initial diameter of the droplets present in the air. It was seen that the size of the droplets has a large impact on the time required for the relative humidity induced condensation process (Figure 30). By reducing the size of the droplets from 20 microns to 1 micron, the duration of the process was reduced from 5 seconds to 12 milliseconds. This means that a portion of the droplets that are seen in actual icing scenarios, which are as small as 6 microns, are much more vulnerable to the relative humidity effects as compared to larger droplets. As a result of this variation in vulnerability, the droplet spectrum is further smoothed such that the smaller droplets grow in size.

Regardless of the total time requirements of droplets with different radii, for every chunk of air passing through the supersaturated regions, there will be some level of condensation. This can accrete to considerable amounts when integrated over a long exposure. In order to make a rough estimate of this effect, the numerical box experiment approach was used with some modifications for flight at $5000 \mathrm{~m}$ standard altitude with $R e_{c}=1 \times 10^{6}$, and 21 inches of chord length. For reference length, the portion of the 


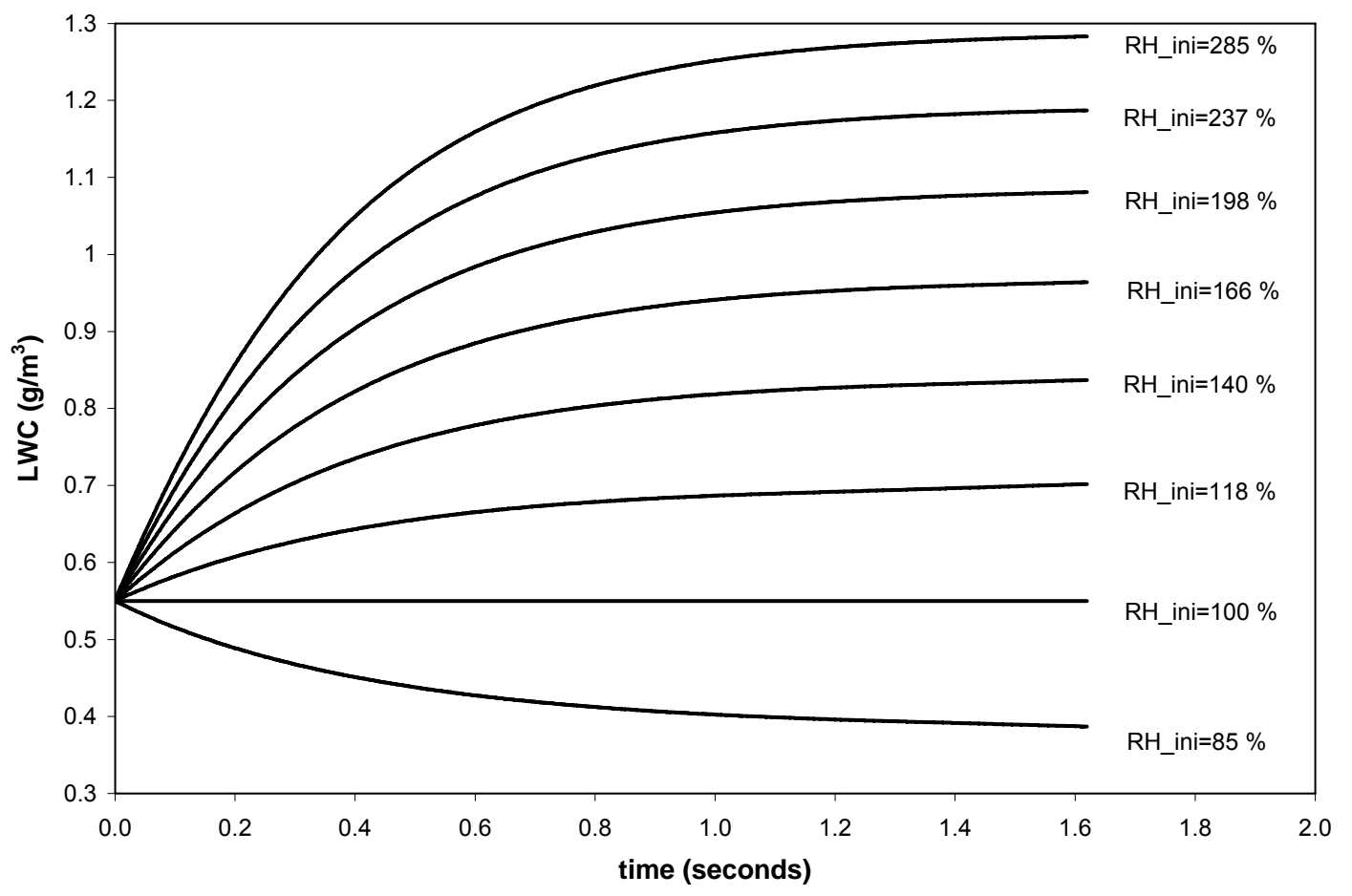

Figure 29. $L W C$ history for different initial relative humidity levels.

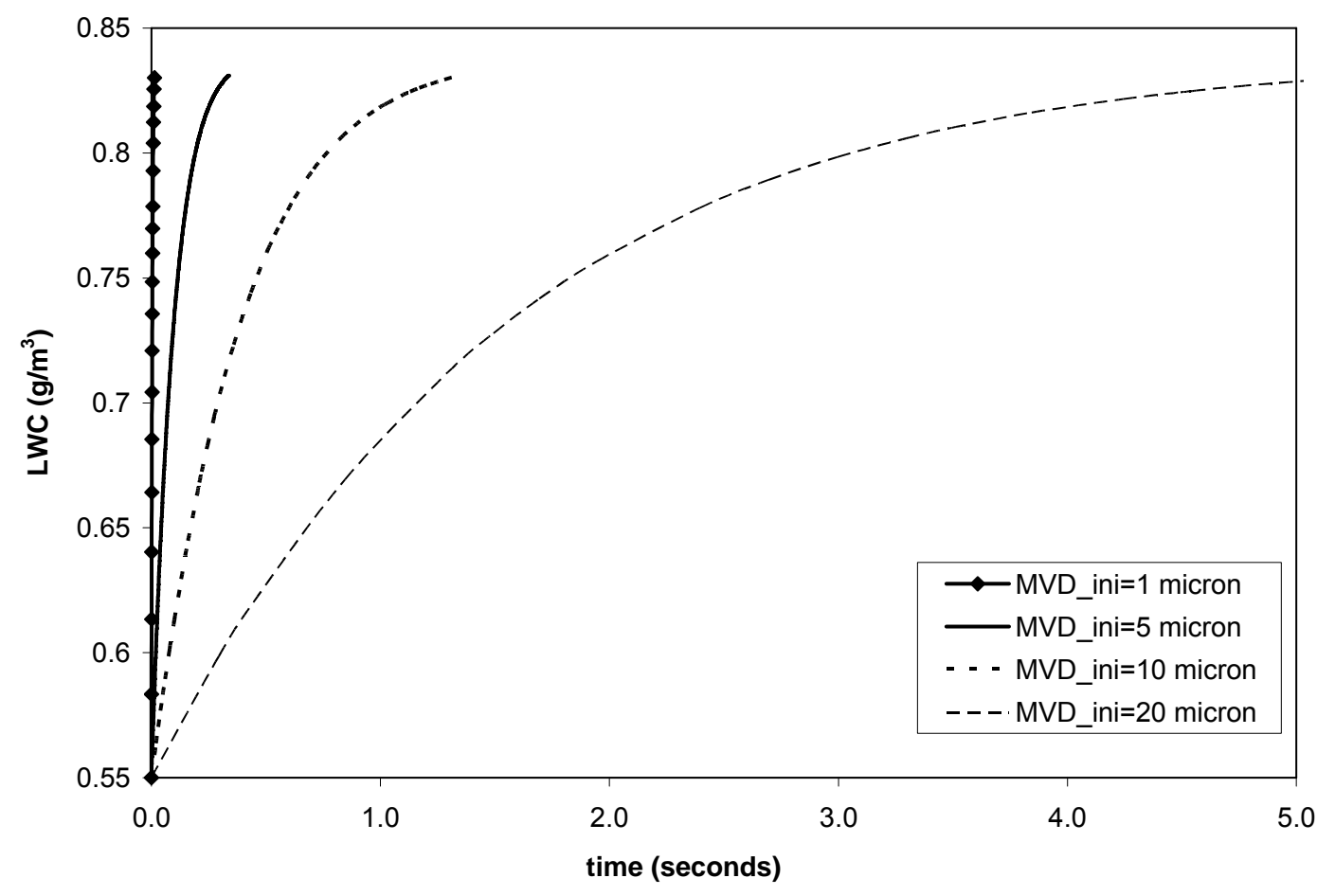

Figure 30. $L W C$ history for different initial $M V D$ 's; imposed initial $R H_{w}=140 \%$. 
chord between $10 \%$ and $30 \%$ chord locations was chosen, simply because this area is typically under very low pressure. XFOIL program was used to calculate the pressure distribution around a NACA 0012 airfoil at $4^{\circ}$ angle-of-attack. Then, the average pressure was calculated in the mentioned portion of the airfoil both on the upper and on the lower surface, separately. A rough estimate of velocity for this average pressure was calculated using the Bernoulli equation. This velocity along with the reference length of $20 \%$ chord gives an estimate of the residence time for the droplets in this supersaturated region. Within the given residence time, the change of $L W C$ was determined by checking the $L W C$ history in the box. Once the residence time is complete, the region is refreshed by the next volume of air, which again stays for the same amount of residence time. This procedure continues until a prescribed accretion time is over. The condensation is calculated for surface roughness elements that have the initial radius of the droplets in the air. This means that there is an initial level of roughness on the surface caused by, say, frozen droplets that impinged the surface earlier. Although the air is refreshed, the height of the roughness elements continuously increases due to the continuing condensation. The results of this numerical experiment are presented in Figure 31 for the upper surface $\left(C_{p, \text { average }}=-0.72\right)$, and in Figure 32 for the lower surface $\left(C_{p, \text { average }}=-0.05\right)$.

Both in Figure 31 and Figure 32, it is seen that the initial rate of growth is higher for the roughness elements with smaller radii. However, as they grow in size, the growth rate of all roughness elements reach about the same value. Nevertheless, initially the larger roughness elements continue to be the largest. Over a 30 minute period, the roughness elements on the upper side, which were initially 50 microns in diameter, grew to a diameter of 90 microns. This level of roughness is sufficient to cause an increase in drag; for further extended exposures, it is likely to form new impingement surfaces beyond the direct impingement region on the leading-edge. On the lower side, due to a lower degree of supersaturation, the condensed amount was less, as reflected by the 5 to 10 micron increase in the diameters of the roughness elements located therein. In the previous literature, roughness at this level $\left(k / c=10^{-4}\right)$ is associated with decrease in maximum lift as much as $13 \%$ and increase in drag up to $40 \%$ 37,38 .

As an interim conclusion, it can be said that within the direct impingement region, the effect of the relative humidity is not likely to be seen, because the high amount of water coming with the large droplets will suppress the effects manifested due to relative humidity. However, the areas that are out of direct impingement, e.g. rear side of large roughness elements and suction surfaces beyond the direct impingement limits, are still subject to supersaturation for prolonged periods. So, it is likely that condensation and frost growth are going to be induced due to relative humidity in these regions. 


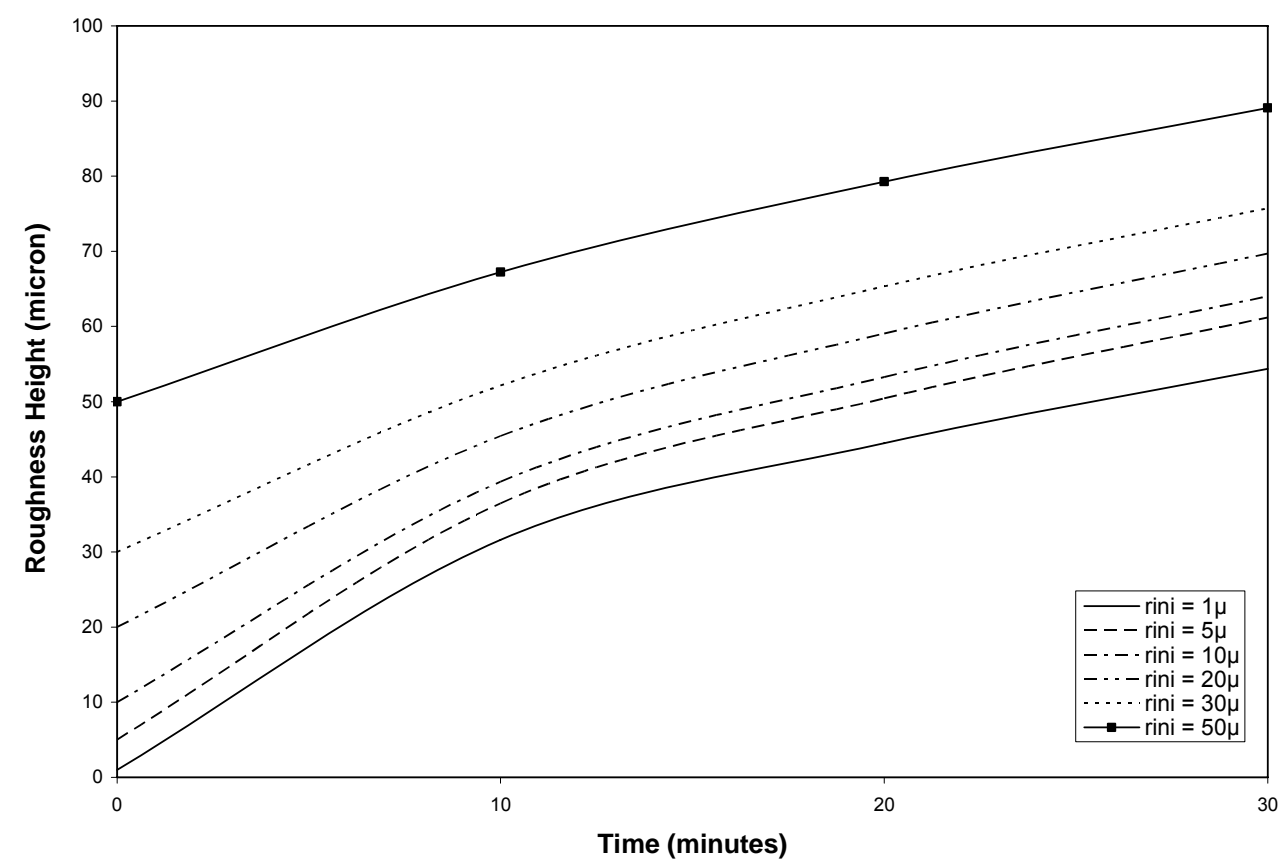

Figure 31. Change in roughness height due to relative humidity on the upper side of a NACA 0012 airfoil in flight with $R e_{c}=1 \times 10^{6}$ at $5000 \mathrm{~m}$ standard altitude.

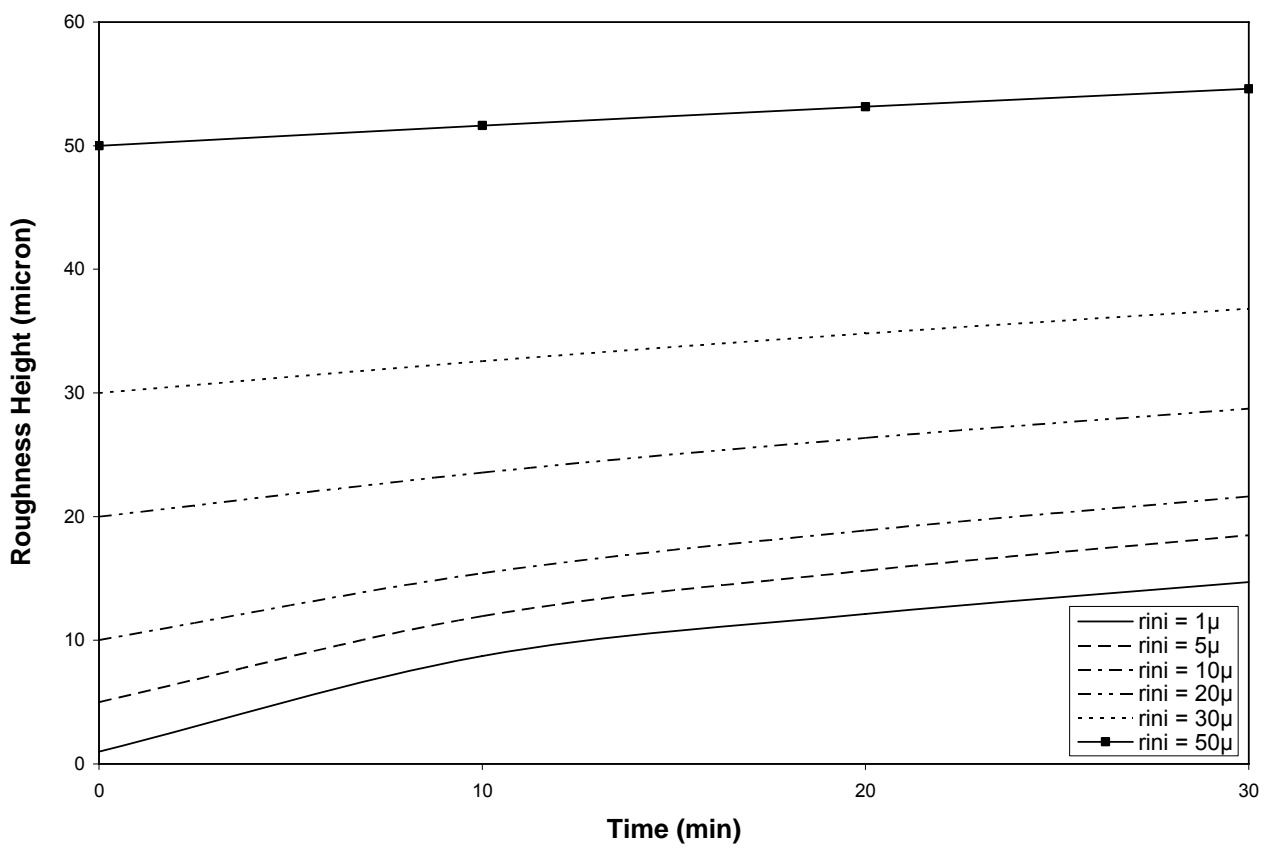

Figure 32. Change in roughness height due to relative humidity on the lower side of a NACA 0012 airfoil in flight with $R_{c}=1 \times 10^{6}$ at $5000 \mathrm{~m}$ standard altitude. 


\section{Vortex Interaction}

The vortices shed from existing leading-edge ice roughness are expected to have two modes of impact on the downstream ice growth as described below.

\section{Droplet paths}

In typical ice accretion codes, droplets are subject to the flow induced by the potential flow coupled with a boundary layer solver. This type of solver is not capable of capturing the unsteady separation and vortex dynamics, and the resulting interaction with the flow. The unsteady Navier-Stokes solver used in the present study is capable of capturing large scale and small scale flow features, including separation bubbles and vortex shedding. The impact of the vortex dynamics is that they may alter the path of the droplet; a droplet may impinge on a location other than the one predicted by the potential flow solver, or a droplet that is calculated to miss the surface may impinge. An example of this phenomenon was investigated for an unsteady flow over a NACA 0012 airfoil with glaze ice (Figure 14). Although in the droplet tracing process, the unsteady flow field is used, the stream function contours at a snapshot in time from the vortex shedding process is given in Figure 33 to give an idea about the level of activity in the aft region of the glaze ice.

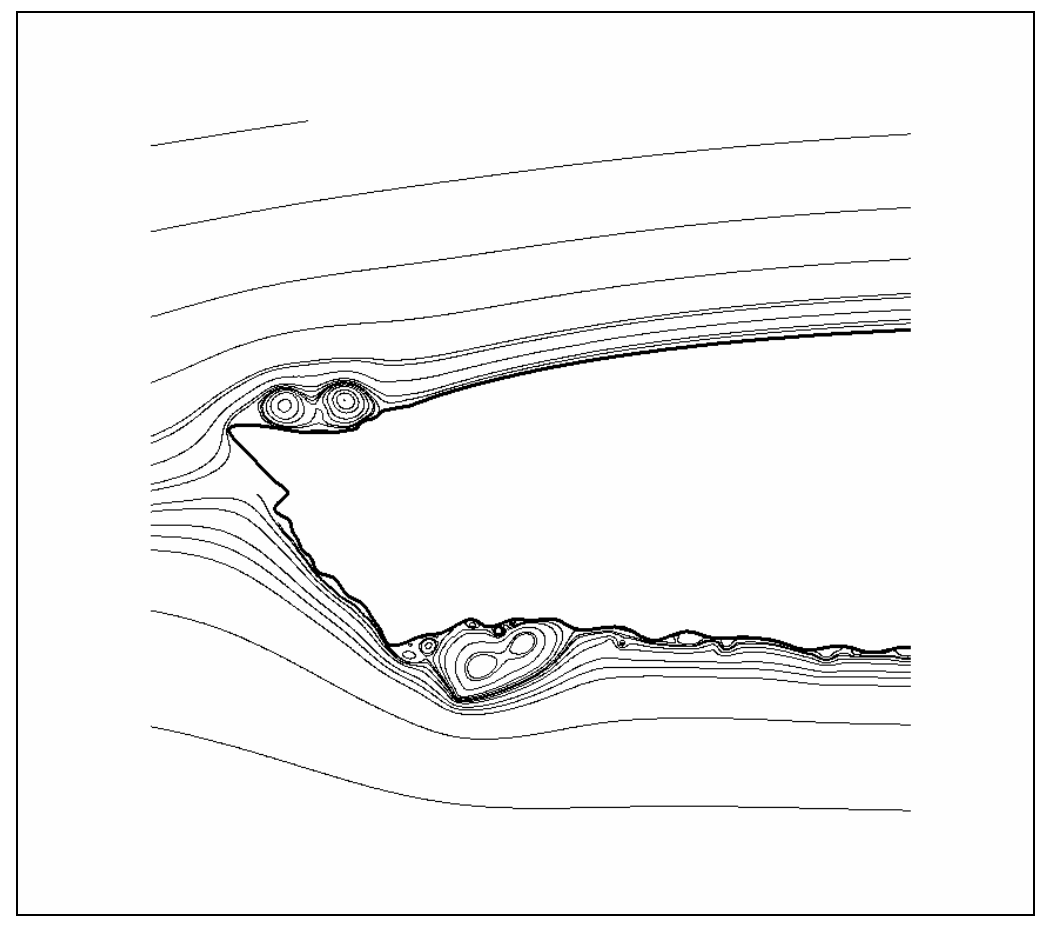

Figure 33. Stream function contours around a NACA 0012 airfoil with glaze ice showing the shed vortices. 
A parametric study of the effect of droplet size was made using this unsteady flow. The droplets with varying diameter were traced in a flow at $5 \times 10^{5}$ Reynolds number for a 21 inch chord length. The smallest droplets had 5 micron diameter. The first set of droplets released into this unsteady flow followed the streamlines very closely so that none of them impinged on the surface (see Figure 34). Even the droplets that were heading for the frontal area of the glaze ice slowed down and followed the local air, no matter how curved the resultant paths were. Especially, the droplet that was approaching the stagnation region exhibited a very interesting behavior. It slowed down with the local air so much so that within the given time it could not get out of this region. This observation gives a clue about the possibility of relatively small droplets being captured by some local flow features such as entrapped vortices.

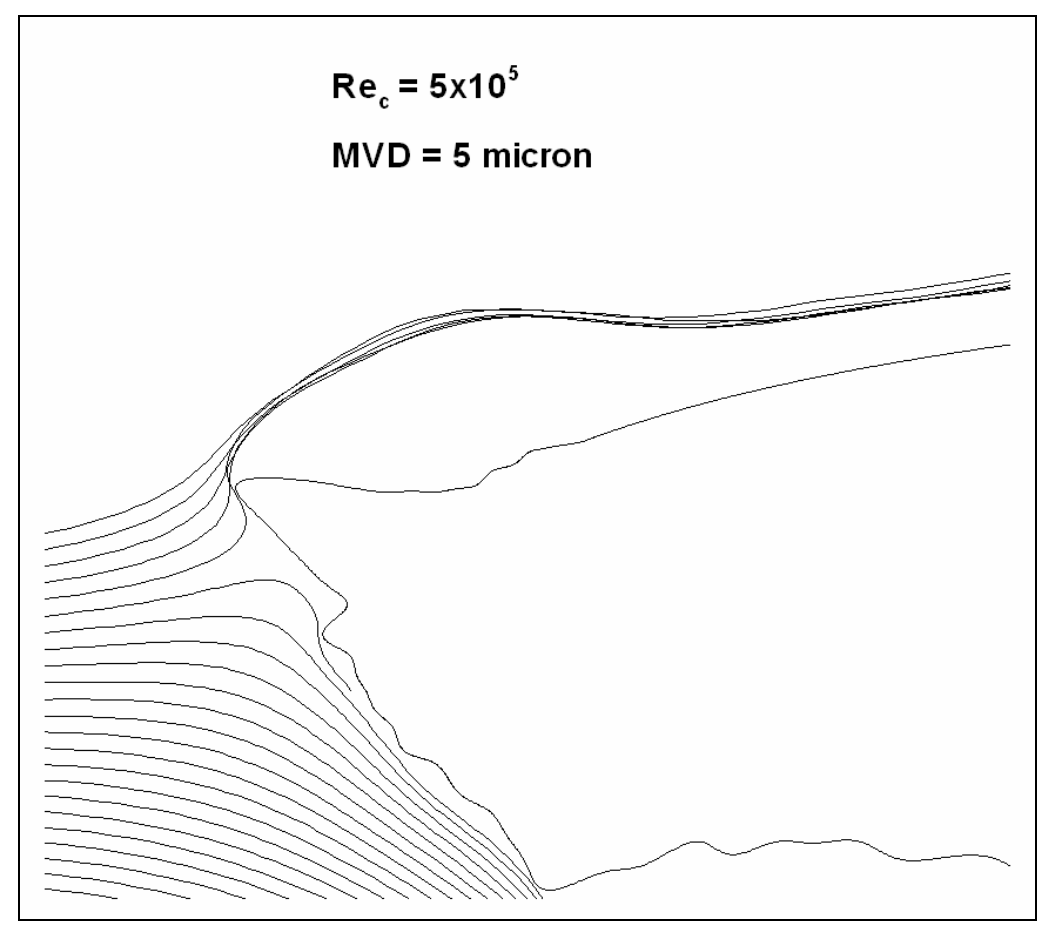

Figure 34. Paths of the droplets ( $M V D=5$ micron) that are released simultaneously in an unsteady flow around a NACA 0012 airfoil with glaze ice.

The paths of all of the impinging droplets during the tracing period are superposed and are provided in Figure 35 through Figure 38. For the 20 micron case (Figure 35), the vortex behind the glaze horn causes strong divergence from the regular path, because the inertia of the droplet is low enough that its path is easily manipulated. The same trends were observed for 30 micron (Figure 36) and 40 micron (Figure 37) droplets at different phases of the vortex shedding. However, for the 50 micron case, the shed vortices were never strong enough to cause severe change in path, though they still caused slight deviation leading to impingement. 


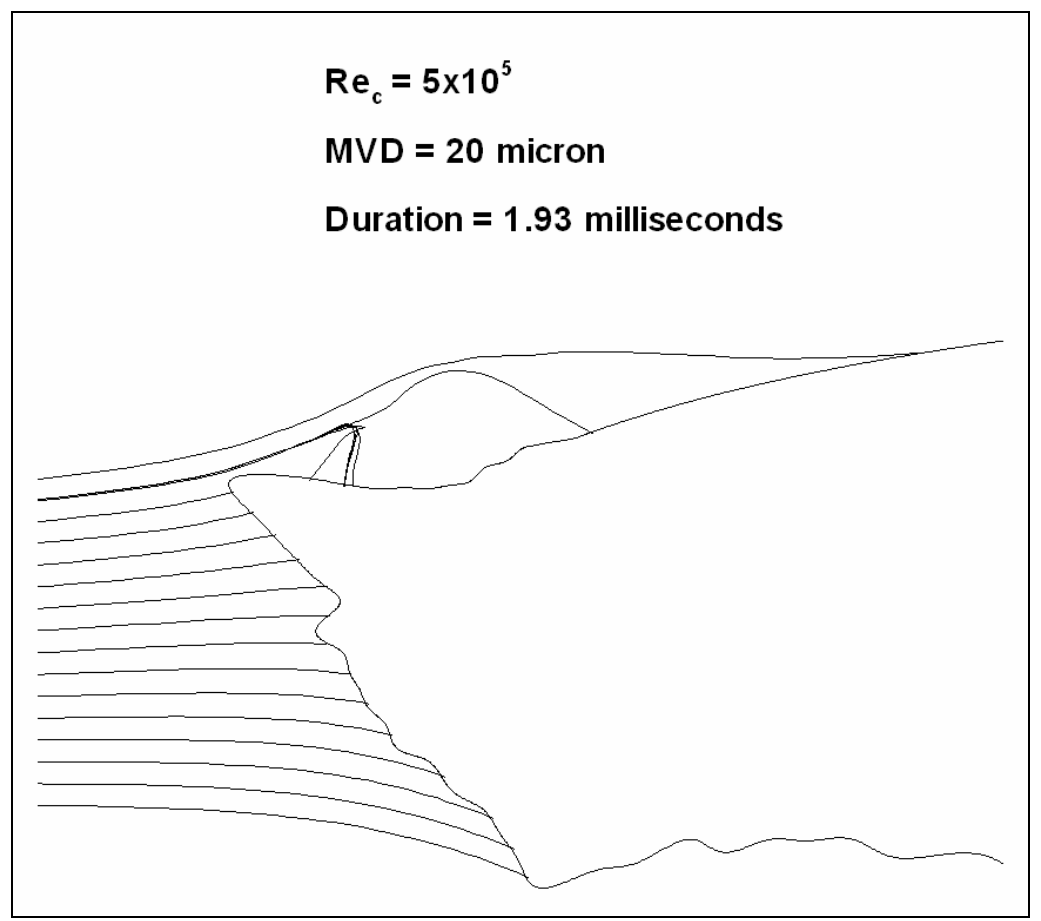

Figure 35. All droplet impingement paths (MVD=20 micron) in an unsteady flow around a NACA 0012 airfoil with glaze ice.

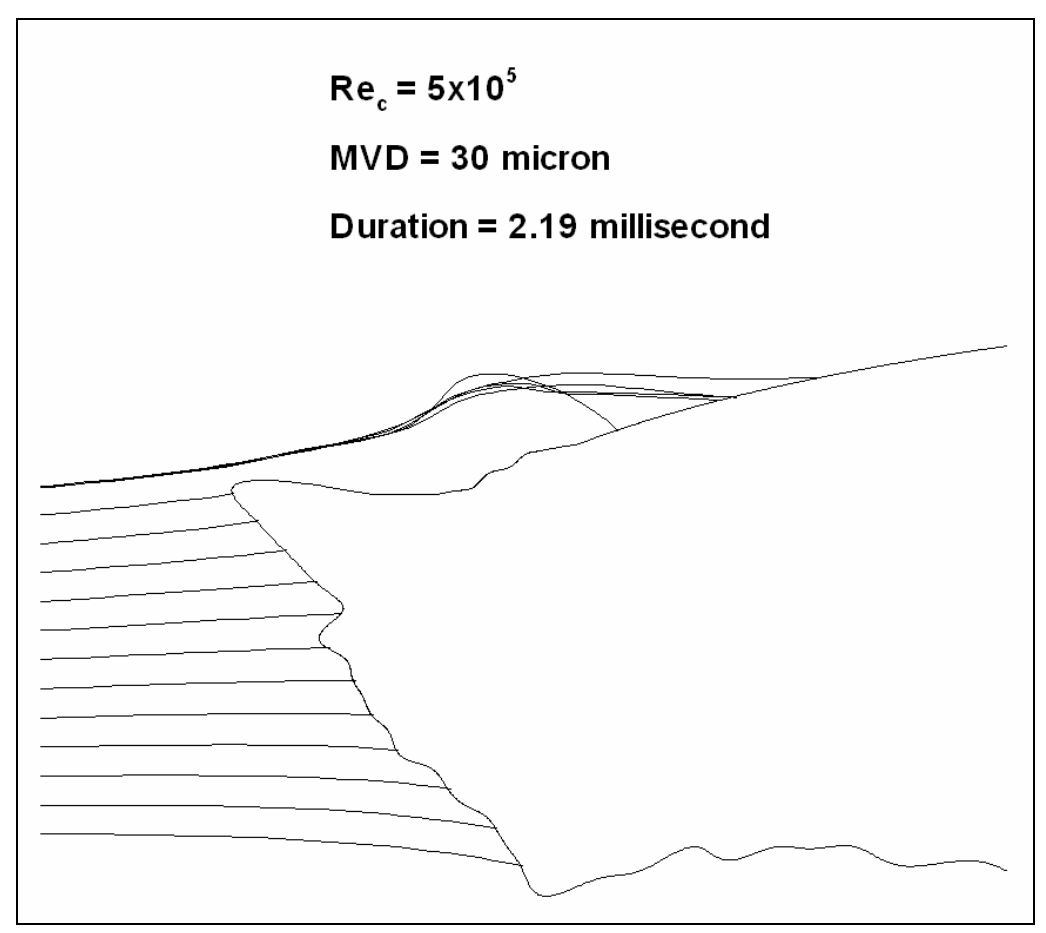

Figure 36. All droplet impingement paths (MVD=30 micron) in an unsteady flow around a NACA 0012 airfoil with glaze ice. 


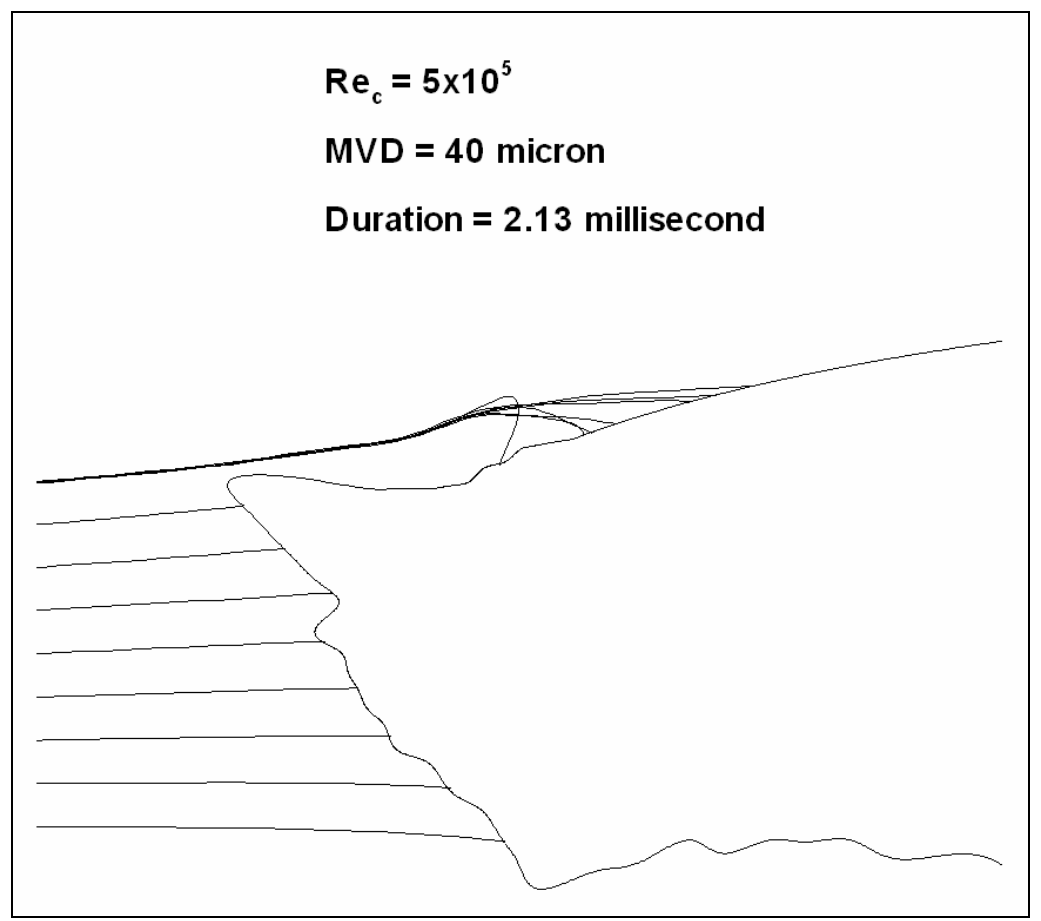

Figure 37. All droplet impingement paths (MVD=40 micron) in an unsteady flow around a NACA 0012 airfoil with glaze ice.

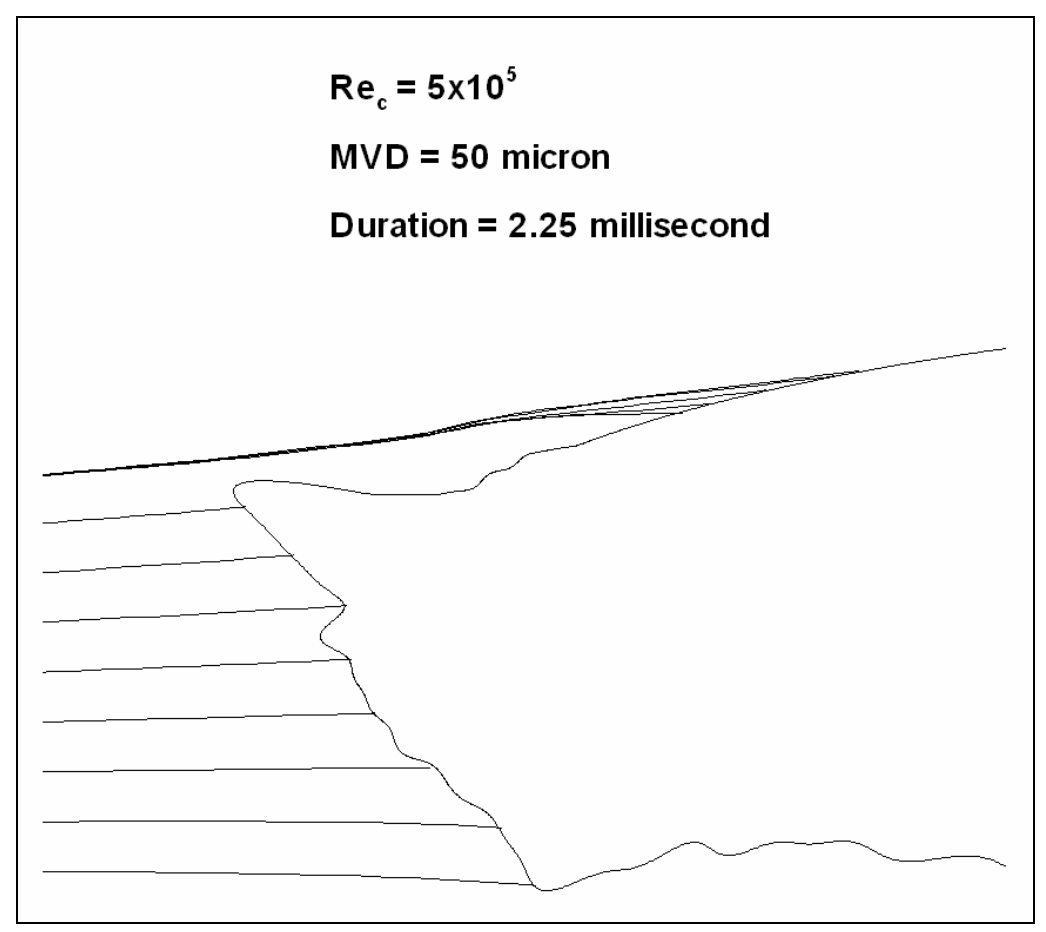

Figure 38. All droplet impingement paths (MVD=50 micron) in an unsteady flow around a NACA 0012 airfoil with glaze ice. 
At each designated time step, certain number of droplets were released from the same initial locations. The designated time steps were chosen such that the distance between the neighboring droplets are the same both vertically and horizontally in the approaching flow. Although the consecutive set of droplets were released from the same initial locations, the paths of the droplets that are released from the same point at different time steps were not necessarily the same if they interacted with the unsteady features; otherwise the paths of the droplets with the same original location remained the same due to localized steady flow. Below is a further discussion on this matter.

For the droplets that impinge the frontal area of the ice shape, the paths due to successive droplets from the same original point overlap; because they are not interacting with the unsteady features of the flow. For this reason, in the tracing pictures, the paths of several consecutive droplets heading the front area overlap and are not distinguishable. It is important to note here that in an actual case, the droplets would be distributed haphazardly with some distance between them, rather than aligning themselves on a grid. Although unsteady features are not coming in to the picture in this frontal area, due to the haphazard droplet distribution in an actual case, the droplet impingements thereon would have a distribution along the front surface. As for the droplets that do not impinge the front area and penetrate into the rear regions of the glaze ice, they do interact with the unsteady features of the flow. Therefore, as far as their path to their end impingement locations in the rear region, the droplets released from the same location do vary due to unsteady effects. The essential point in these tracing summaries is that the rear impingements would not be seen by current icing codes that rely on potential flow for droplet tracing, hence the extra ice growth beyond impingement limits.

One conclusion that can be drawn by comparing these observations is that the inertia of the droplet must be relatively low compared to the strength of the vortices so that their effects can impact the subsequent paths of the droplets. The two extremes of the droplet inertia-vortex strength comparison are droplets with very high inertia and the droplets with very low inertia. If the droplet's inertia is extremely low, then it is going to follow the streamlines at all times; therefore it not going to impinge the surface regardless of the curvature of the streamlines. The other extreme is the droplets with extremely high inertia. For this extreme case, the result is that the droplets are not affected by the flow features at all; a result that can be deduced from the comparison of the tracing summaries. Therefore, for a droplet to exhibit the vortex interaction effects, it must have low enough inertia to get into the interaction with the vortices, but at the same time high enough inertia to end up with an impingement with the surface.

The statistics of the interaction of the droplet paths with the unsteady vortex shedding is presented in Table 2. The table shows the $M V D$ of the droplets that were used in the simulation along with the 
corresponding $L W C$ in the air. The number of impingements recorded on the frontal area of the main ice shape and on the rear side of it are also presented in the same table. The frontal impingements are the ones that can also be captured by the particle tracing programs that are based on potential flow calculations, because they do not involve interaction with the induced vortices. However, the rear impingement events cannot be captured by typical ice accretion codes, since there is a clear interference due to the vortices.

\begin{tabular}{|c|c|c|c|c|}
\hline MVD & Duration & \multicolumn{2}{|c|}{ Number of Impingements } & Extra Thickness in 7 minutes \\
\hline (micron) & (milliseconds) & Front & Rear & (mm of water for 0.1c, unit span) \\
\hline 20 & 1.93 & 294 & 7 & 0.06 \\
\hline 30 & 2.19 & 216 & 5 & 0.09 \\
\hline 40 & 2.13 & 108 & 7 & 0.21 \\
\hline 50 & 2.25 & 88 & 5 & 0.24 \\
\hline
\end{tabular}

Table 2. Statistics of the interaction of the unsteady vortex field with the droplet paths.

In order to make a rough estimate of the corresponding extra thickness for the accumulated water, an exposed surface area was chosen with dimensions $1 \mathrm{~m}$ along wing span and $10 \%$ chord length $(c=21$ inches) along the chord. The reason for choosing $10 \%$ chord length is that in icing experiments, the minor ice roughness extends around 10-15\% chord length behind the main ice shape. Using the number of extra impingements in Table 2, total number of impingements over a 7 minute period was calculated, with the assumption that the acquired statistics provides a good enough sample for the actual physics. The reason for choosing 7 minutes as the exposure time is that in most ice accretion experiments, this duration is used as the total accretion time. The amount of water accreted due to the extra impingements in this period was obtained by multiplying the total number of impingements with the volume of a single droplet. Then this total volume of water was assumed to be spread over the surface area mentioned above.

The case with 20 micron $M V D$ had the least impact with $0.06 \mathrm{~mm}$ of extra water layer. This was mainly caused by two reasons: the relatively smaller volume of the droplets resulting in considerably less mass per droplet, and their relatively low inertia that helps the droplets follow the streamlines rather than impinge the surface. As bigger droplets were introduced to the flow field, both the water content and the inertia of the droplets increased dramatically; since both are related to mass which increases with the cube of the diameter. The result was that the larger droplets were influenced less by the flow, which decreased the number of impingements. But, their contribution per impingement increased significantly. The highest level of impact came with the 50 micron droplets with an extra water layer of $0.24 \mathrm{~mm}$. 


\section{Frost growth}

The suction surfaces of the wings have low pressure, which make these surfaces subject to supersaturated air. The vortices shed from the ice roughness impose a low pressure on the surface, which enhances the level of exposure to supersaturation. The excess humidity in the air can condense onto the surface as liquid water or direct deposition to frost. However, this effect is only significant in the regions out of direct impingement or regions downstream of existing primary ice roughness. A long exposure time is also needed to see a significant effect.

Another factor affecting the frost growth is streamwise vortices that have the potential to change the pattern of frost formation on the surface ${ }^{39}$. At the locations where the vortex induces a flow onto the surface, the frost formation is faster, whereas at the locations where the vortex induces a flow away from the surface, the frost growth is slower. This can also be traced back to localized changes in the residence time of supersaturated air over the exposed surface. The location, where a flow towards the surface is induced, simulates a stagnation flow, and the speeds are lower in such locations. This leads to increased time for the deposition to take place. On the other hand, the opposite effects are observed at locations where a flow from the surface is induced by the vortex, leading to decreased time for the deposition to occur.

Aside from the local variations in the residence time of supersaturated air over the exposed surface, the character of the surface is also important for the time required by the deposition process. A rough surface is more susceptible to frost growth by virtue of its surface characteristics that provide more and better nucleation sites compared to a clean, smooth surface. Therefore, frost growth is an induced phenomenon by the primary parts of the ice accretion over the wing.

\section{DISCUSSION}

The flow field and the pressure calculations around airfoils with ice accretions have shown that the local air flow can become highly supersaturated. However, due to the small time scales involved in the flow, the impact of relative humidity is not a first order effect in the areas of direct impingement, where high amounts of water are collected due to the impinging droplets that are as big as 30 microns in diameter. For the areas out of the direct impingement region, given a sufficient amount of exposure time, relative humidity can result in significant level of small-scale roughness, which will increase the drag. It was also seen that the vortices shed from the ice surface interact with the droplets and can alter the trajectory. This allows for a potential droplet impingement in locations that are out of direct impingement region. The existence of these strong vortices depend on the height of the roughness preset on the airfoil 
surface. Therefore, as the main ice shape grows, the vortices that are formed are going to be relatively weak and so, are going to affect droplets with small diameters. As the ice shape grows further, the vortices induced by it are going to be stronger and will be able to modify the paths of larger droplets.

It is important to note here that in a fully 3D case, there will be a more complex vortex structure that is going to impose a different impingement distribution for the droplets in the mentioned aft regions. A possible speculation at this point is that in the regions of supersaturation, the condensed water can be swept by the shed vortices towards the leading-edge. Considering that ice feathers grow outside of the impingement regions and they tend to extend towards the leading-edge as they grow, it is possible that the vortex-sweeping is a contributing factor to the feather growth, an effect that is not addressed in current ice accretion modeling. 


\section{CHAPTER V}

\section{FURTHER DISCUSSION ON FROST GROWTH}

\section{INTRODUCTION}

In general, frost growth is mainly an issue for the designers of cooling or air conditioning devices. The reason for such concern is the fact that a frost formation over the heat transfer surfaces degrades the rate of heat transfer between the air passage and the cooling unit. As a result, the air is not cooled as effectively, and due to the increased level of drag generated by the rough surface of the frost, the power requirement of the unit increases ${ }^{40}$. Many researchers are trying to come up with an accurate frost formation model that would computationally predict any frost growth on a given surface under the specified conditions. However, this task is not as easy as it sounds. The complicated character of the frost geometry (both at its surface and within), the phase change issues and the unsteady three dimensional flow features result in a hard-to-model phenomenon.

The easiest way of modeling the frost growth is done by an unsteady one-dimensional formulation with some assumptions ${ }^{41}$. This method of modeling assumes a uniform frost distribution along the given surface, but it tries to predict the growth and the densification of the frost by time. There are several aspects of heat transfer in this analysis: the heat transfer due to phase change from vapor to ice, heat transfer between the air flow and the frost, and the heat transfer between the base surface and the frost. Along with the heat transfer issues, the distribution of the temperature within the frost is another factor that needs to be addressed.

Aside from the heat transfer aspects of the frost growth, there is also the mass transfer aspect, which causes the growth of the frost by phase change from vapor in the air to ice on the frost layer. In a previous experimental study, the Reynolds number, which is a very common factor in mass transfer, was not found to be significant for the frost growth ${ }^{42}$. Since this process is taking place at the molecular level, the very beginning of the frost growth process and the later parts of it do not have the same characteristics in terms of the microstructure. The difference in the microstructure causes a difference in the growth rate. The very beginning parts of the process involve a period that is modeled by crystal growth; but, in the later parts of the process, the crystals emerging from the surface become large enough to form surfaces to pick up more moisture and to catch the extremely small droplets in the air, resulting in a faster growth rate. Both periods of the process have been experimentally analyzed, and semi-empirical models were developed that predict the growth rate of frost under suitable conditions ${ }^{40}$. 
Until recent years, it was thought that the moist air becomes saturated just above the frost layer ${ }^{43}$. Many models have been developed and were utilized in the design of many cooling devices. These models, with good performance for small periods of exposure, experienced inaccuracies in terms of the long-time exposures. One recent study showed that this assumption of saturation was not correct and that there is actually a supersaturation in the moist air just above the frost layer ${ }^{44}$. This conclusion comes from the fact that the water molecules have to overcome the Gibbs energy (surface energy) barrier in order to join the frost that is in solid phase. This requires an extra accumulation of energy, which is obtained by having supersaturation conditions over the frost surface. The model developed from this method gave better results. However, the three-dimensional, unsteady frost growth process is still a phenomenon that needs further research.

In the present study, the main frost characteristics such as temperature distribution within the frost and the growth rate of the frost under extremely high supersaturated conditions are investigated. This investigation was motivated by the findings from the previous studies that showed an extremely high level of supersaturation on the parts of the wing where there is suction (see Chapter IV). Another motive was to compare the growth rate of the free droplets in the air to the frost growth on the wing surface. As shown in the chapter IV, the time scales for the relative humidity effects required by very small droplets that are on the order of 10 microns or less are much smaller than the time scales required by the droplets that are on the order of 100 microns. Since the droplet growth and the frost growth are both fed by the moisture in the air, there must be congruence between the two.

The key findings showed that the time scales required by the frost growth under extremely high supersaturation conditions are still on the order of hours. This means that the wing surface must be exposed to the moist air for extended hours in order to accrete a significant mass of frost. However, it must be noted that this result is based on a growth model that solely depends on vapor deposition on a surface that is exposed to moist air flow at low speeds. Therefore, if the growth process is coupled with some other growth mechanism such as droplet catch, then the required time scales would likely drop. The second part of the study, which compares the droplet growth in air to the frost growth on solid surface, showed that as the initial droplet radius is increased to the order of 100 microns, the time required by the droplet for a significant growth in its mass achieves the same level as the frost layer on a solid surface.

\section{GOVERNING EQUATIONS}

Chapter IV includes the formulation that is used to calculate the time required for the relative humidity effects on the droplets in case of a supersaturation. So, here only the frost growth equations are going to be explained. The first step is to determine the temperature distribution within the frost and 
especially the temperature at the frost surface. For this purpose, a 1-D and a 2-D heat transfer problem was solved numerically over a frost layer with a constant height of $2 \mathrm{~mm}$.

$$
\begin{gathered}
\frac{\partial}{\partial x}\left(k_{f r} \frac{\partial T_{f r}}{\partial x}\right)=\rho_{f r} C_{p, f r} \frac{\partial T_{f r}}{\partial t} \\
\text { where } \rho_{f r}=\varepsilon_{f r} \rho_{a i r}+\left(1-\varepsilon_{f r}\right) \rho_{i c e} \text { and } C_{p, f r}=C_{p, a i r} \varepsilon_{f r} \rho_{a i r}+C_{p, i c e}\left(1-\varepsilon_{f r}\right) \rho_{i c e}
\end{gathered}
$$

In these equations, the variation of porosity was selected to be a linear one from the base value of 0.0 to the frost surface value of 0.8 . The frost properties are then calculated by using the equations given above and the physical properties of air and ice. The presented results are at the sea level, because the frost growth is faster compared to higher altitudes. Therefore, the growth rates are going to give an idea about the upper bound for the frost growth on the exposed surfaces.

$$
\begin{array}{ll}
\rho_{\text {air }}=1.225 \mathrm{~kg} / \mathrm{m}^{3} & \rho_{\text {ice }}=917 \mathrm{~kg} / \mathrm{m}^{3} \\
C_{p, a i r}=1005 \mathrm{~J} / \mathrm{kgK} & C_{p, \text { ice }}=1960 \mathrm{~J} / \mathrm{kgK} \\
k_{\text {air }}=0.024 \mathrm{~W} / \mathrm{mK} & k_{\text {ice }}=2.4 \mathrm{~W} / \mathrm{mK}
\end{array}
$$

As far as the boundary condition, the frost layer is assumed to be subject to a tangential flow at 10 $\mathrm{m} / \mathrm{s}$. The convective heat transfer coefficient for the heat transfer between the frost and the air are 10 $\mathrm{W} / \mathrm{m}^{2} \mathrm{~K}$ for the laminar case, and $40 \mathrm{~W} / \mathrm{m}^{2} \mathrm{~K}$ for the turbulent case.

Having determined the temperature behavior within the frost, the frost growth process was modeled with the equations as given in chapter IV and below ${ }^{5}$. This model is based on a one-dimensional unsteady formulation. Its superiority compared to other models is that it uses the supersaturation approach at the frost surface, which is a closer model of the actual frost growth physics ${ }^{44}$.

$$
\frac{d x_{f s}}{d t}=\frac{K_{w}}{\rho_{f r}}\left(w_{a m b}-w_{f s}\right)
$$

In this equation, the $x$ coordinate represents the frost height, $w$, the mixing ratio and $K$, the diffusion coefficient of vapor in air. $K$ value that is used in the current calculations is $0.14 \mathrm{~m} / \mathrm{s}$. The mixing ratio, $w$, is calculated by use of the vapor pressure, $e$, and the local air pressure, $p$, as shown below.

$$
w=0.622 \frac{e}{p-e}
$$


The vapor pressure at the frost surface is obtained with the use of the supersaturation approach given in Ref. 43. This approach first determines the level of the relative humidity at the frost surface, as given in Eq. 67. With the help of the definition of relative humidity (Eq. 68) and the saturation vapor pressure at the frost surface in the approaching flow (Eq. 69), one can obtain the actual vapor pressure at the frost surface.

$$
\begin{gathered}
R H_{f s}=0.808\left(\frac{P_{v, \infty}}{P_{v s, \infty}}\right)\left(\frac{P_{v s, \infty}}{P_{v s, f s}}\right)^{0.657}=0.808\left(R H_{i c e, \infty}\right)\left(\frac{e_{\infty}^{\#}}{e_{f s}^{\#}}\right)^{0.657} \\
R H=\frac{e}{e_{f s}^{\#}} \\
e^{\#}=\exp \left[23.33086-\frac{6111.72784}{T}+0.15215 \ln T\right], \text { where } T \text { is in Kelvin. }
\end{gathered}
$$

\section{RESULTS}

The temperature distribution calculations within the frost involved the air flow at $250 \mathrm{~K}$. Two flow regimes were considered: a laminar case and a turbulent case. For either case, the solutions did not show a significant temperature variation within the frost (Figure 39). The temperature always stays around the same temperature as the base surface, which was set to be constant at $0{ }^{\circ} \mathrm{C}$ for all cases. It is important to note here that this calculation did not involve the effect of phase change at the surface, which would cause a heat release into the frost surface. The main concern was to see the relative importance of the convective and conductive heat transfer. With the result obtained from this first step, it can be said that the heat release due to phase change will not be significant either, because the amount of vapor that is changing phase is very small. Therefore, it can be assumed that with a constant temperature base surface, the frost stays at the same temperature as the base, as long as it is under the freezing point.

The constant temperature at the frost surface was then used in the frost growth calculations. The first step in the growth calculations is to obtain the value of the supersaturation at the frost surface. This is shown in Figure 40 as a function of the frost surface temperature at different ambient pressures. It is clearly seen that lower frost surface temperatures lead to much higher supersaturation levels in the vicinity of the frost surface. It is interesting to see that supersaturation levels as high as $500 \%$ can be obtained at the frost-air interface, if the approaching flow is at $100 \%$ relative humidity (saturated) with respect to ice initially. This level of supersaturation cannot be achieved in the free atmosphere; similar trends were shown in case of a temperature drop at the core of a vortex shed from the ice roughness in 
Chapter III. Both results show that in case of a forced flow, the transient features of the relative humidity field can manifest very different properties compared to free atmosphere conditions. Figure 40 also shows the variation of the supersaturation level at different ambient pressure values. If everything else is kept constant, then an increase in pressure leads to an increase in the partial pressure of the vapor in the air, hence higher supersaturation values.

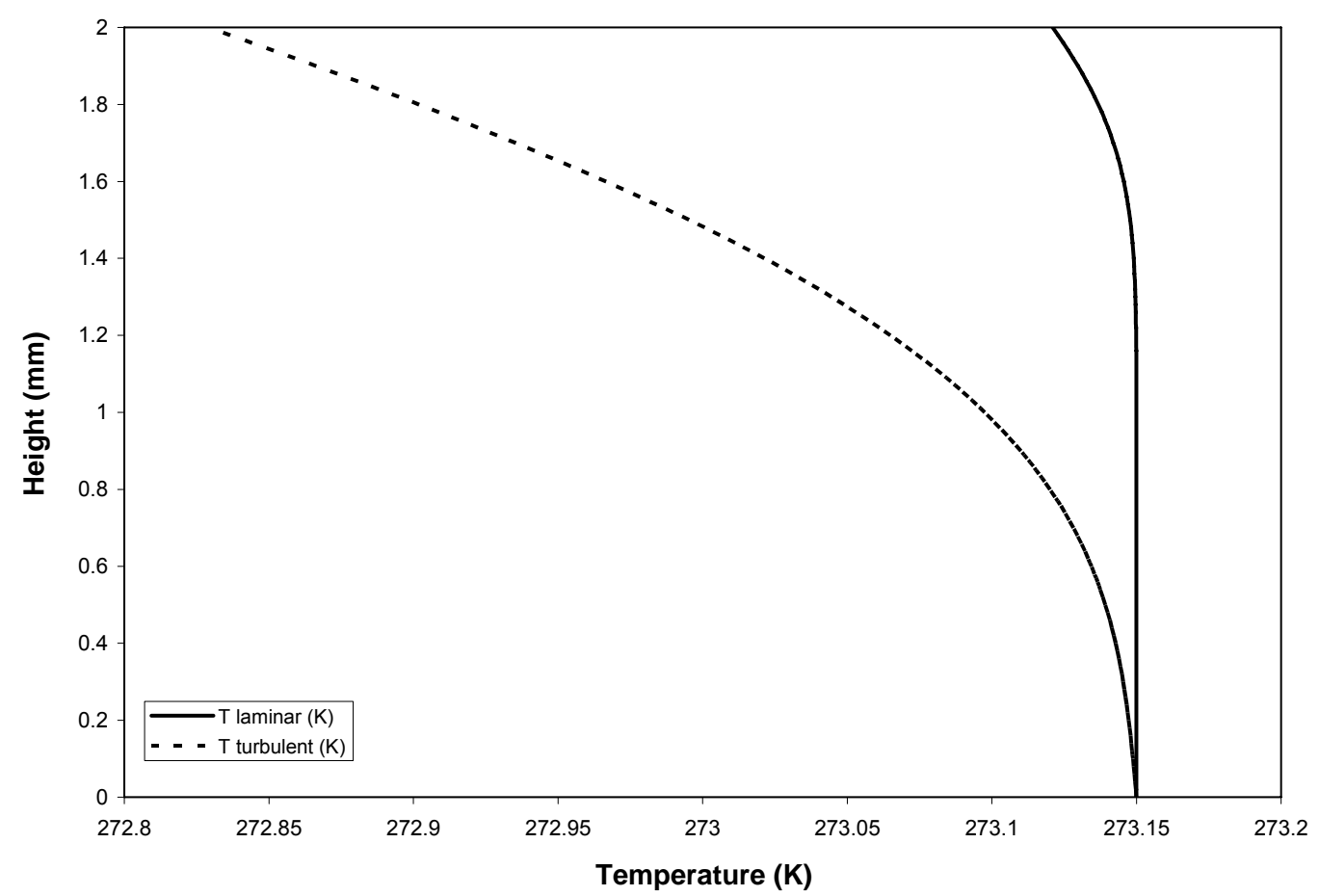

Figure 39. The variation of the temperature within the frost subject to convective and conductive heat transfer.

The same trends are also shown in Figure 41, where the growth rate is shown as a function of the ambient pressure. Different curves were obtained for different porosity level in the frost. Higher porosity leads to less dense frost and a higher frost layer due to enhanced growth rate.

The effect of the frost surface temperature on the growth rate of the frost is shown in Figure 42 with different curves obtained for different porosity levels. As expected, colder surface temperatures lead to higher growth rates. However, the effect of the porosity on the growth rate is more pronounced compared to a drop in temperature, which is also seen in Figure 41. Therefore, the conditions that lead to higher porosity in the frost can severely drop the time required to obtain a significant frost on the airframe surface. 


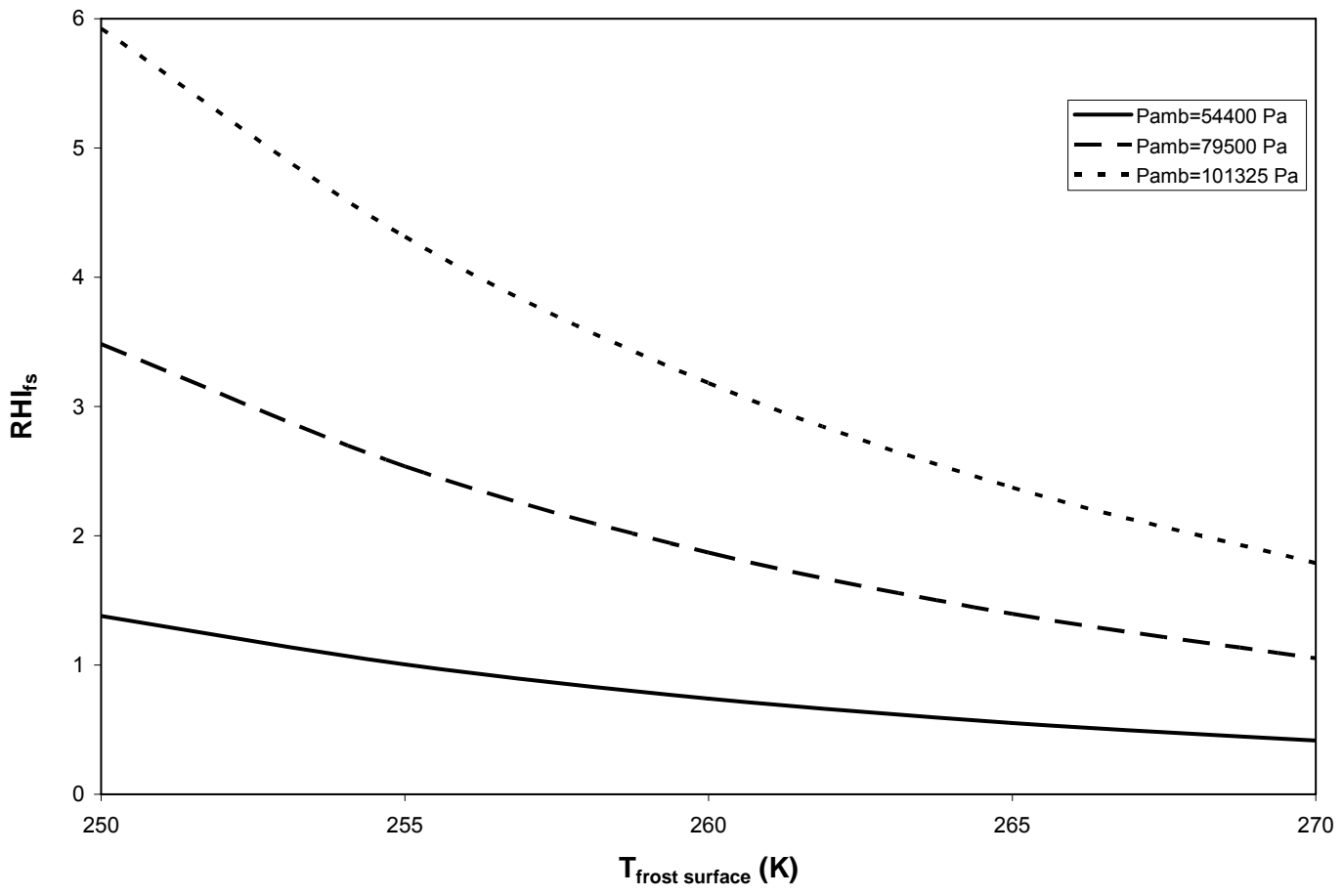

Figure 40. The variation of the relative humidity at the frost surface at different ambient pressures.

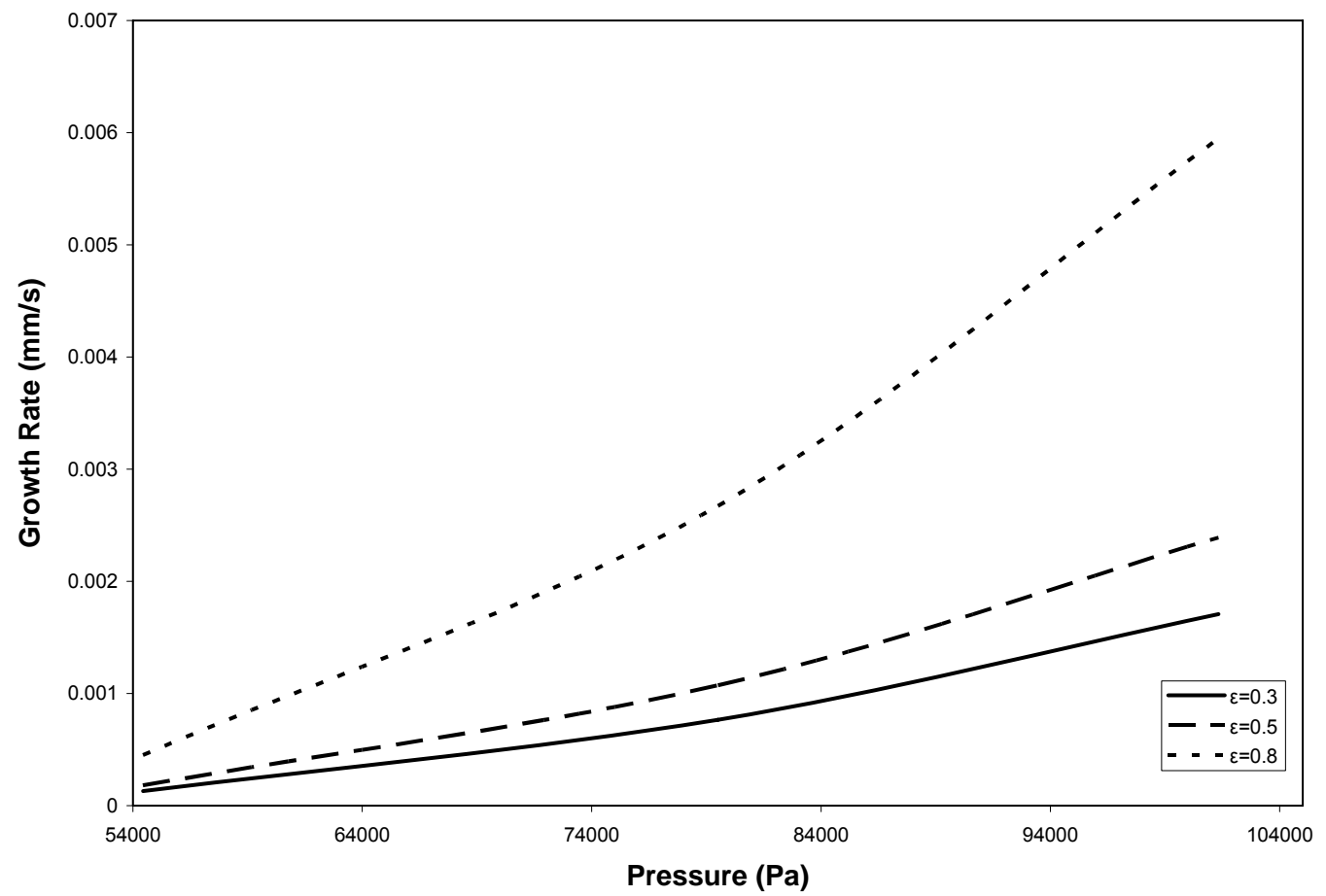

Figure 41. Effect of ambient pressure on the frost growth with various porosity levels at the surface that is kept at $0{ }^{\circ} \mathrm{C}$. 


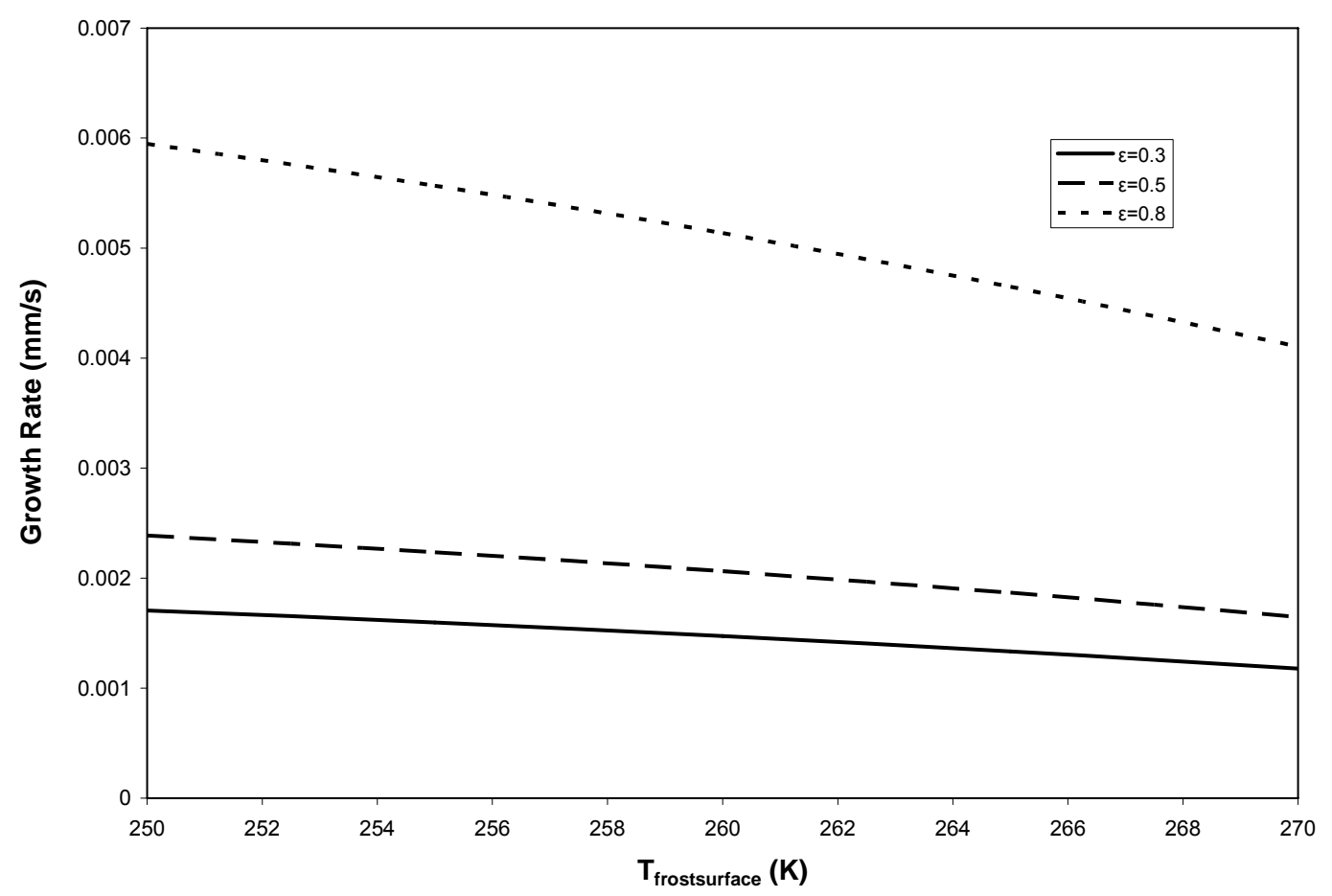

Figure 42. Effect of frost surface temperature at sea level conditions with different porosity levels.

It is important to note at this point that the required time for the formation of frost that has a thickness on the order of millimeters has an order of thousands of seconds. Therefore, several hours of exposure is required for a significant frost layer to form on a surface. However, as far as the droplet growth in case of a supersaturation, the time scales are much less than what is seen for the frost case. Considering that both processes are fueled by supersaturation, should not the time scales be close? The resolution of this discrepancy is the area of exposure to the supersaturated air. In case of droplets, due to their small radii, the net area in touch with air per unit volume of water is considerably large compared to the case of frost, where a flat plate with frost roughness is exposed to the supersaturated air. To show the convergence between the two cases, droplets with much higher surface area, hence larger diameter, were used in the box experiment described in Chapter IV. The results are shown in Figure 43 for several initial droplet diameters. The case with 1000 microns has an equilibrium time requirement about 3 hours, which is on the same order of the frost case. Therefore, it can be concluded that the average effective radius of curvature for the small-scale roughness features present on the frost surface is on the order of millimeters.

\section{DISCUSSION}

This study includes a parametric study on frost growth over surfaces that are subject to extremely high supersaturation values. The results from this study showed that the frost growth is favored by a 
lower surface temperature and a higher ambient pressure. Also the time required to get a significant mass of frost was found to be on the order of hours. This long period is thought to be due to the reduced area of contact between the moist air and the frost surface, which is a conclusion that can also be obtained from very large droplet growth scenarios in free atmosphere. The velocity chosen for the air flow was much lower compared to an actual flight velocity. The reason for this is that the frost growth formulae were given for air conditioning devices that are subject to low velocities. Therefore, frost growth in case of flight velocities were out of range. Therefore, frost growth under flight conditions still needs an in-depth experimental and theoretical analysis.

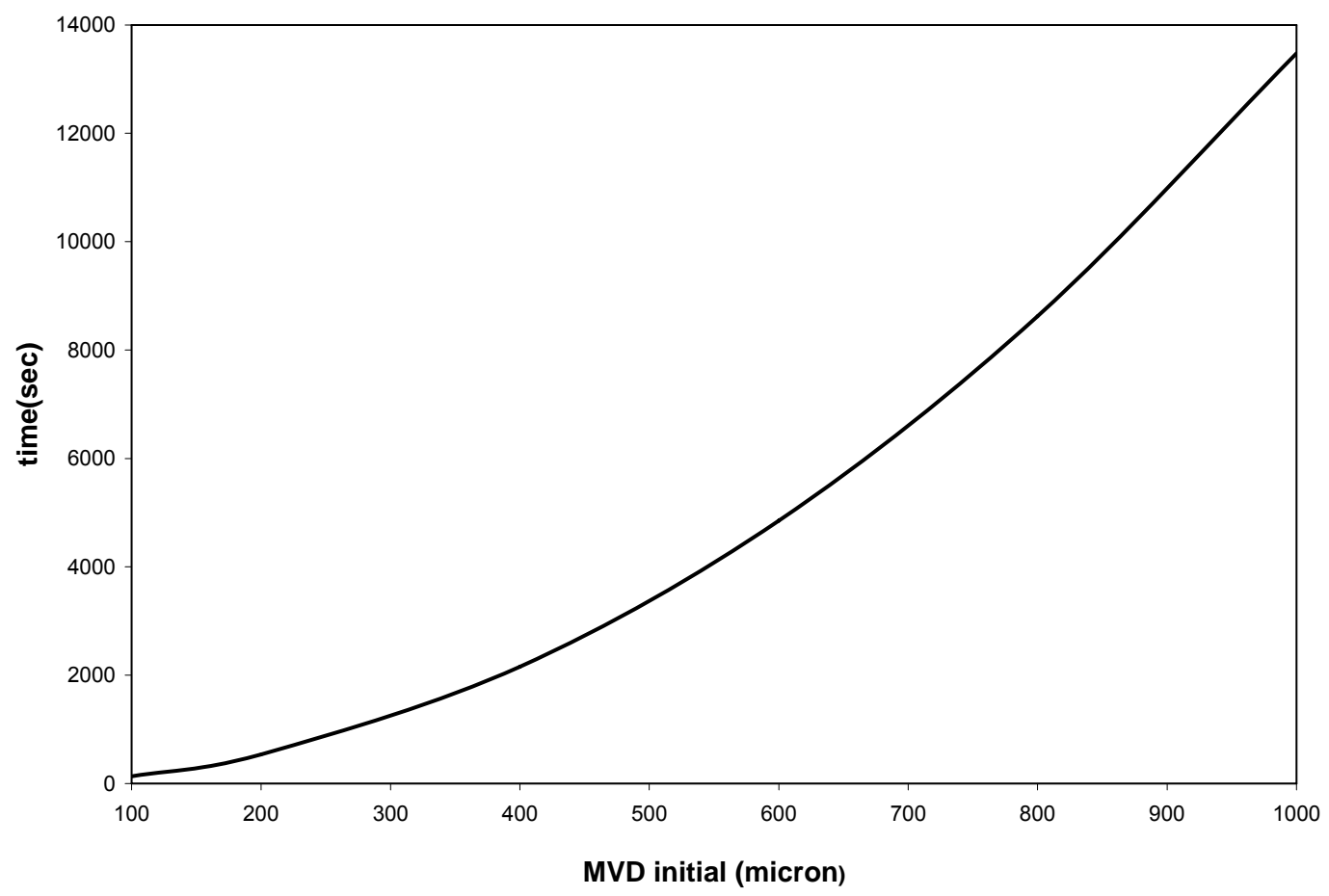

Figure 43. Change of time required to return to the saturation conditions as a function of initial droplet diameter. 


\section{CHAPTER VI}

\section{CONCLUSIONS}

This Ph.D. study focused on two main alternative mechanisms for downstream growth of ice shapes over the critical aircraft surfaces. These mechanisms are the relative humidity induced ice/frost growth, and the droplet path change due to interaction with the vortices induced by the existing ice shape. The relative humidity field in the airflow over the ice shape is affected by the vortices that are shed thereof, which have low-pressure cores and which induce low pressure on the airframe surface. Therefore, both the relative humidity related mechanism and the vortex interaction mechanism are consistent with the idea that the ice shape has an impact on its own growth.

It was shown that the suction areas around the airfoil with or without ice accretion are subject to very high levels of supersaturation. This high level of supersaturation is due to the forced and transient response of the relative humidity field to the sudden appearance of the conditions around the wing. So, the conditions around the wing are suitable for a frost growth and/or changing the droplet diameter as they pass through these highly supersaturated regions. For a particular case considered in this study, given enough time, relative humidity could increase the $L W C$ as much as $100 \%$, or decrease it $30 \%$. However, the time scales over a wing leading-edge is much smaller than the scales required by relative humidity effects. Therefore, a portion of the full effect is going to be seen on the exposed surfaces. For a particular case, a rough estimate of $0.4 \mathrm{~mm}$ of increase in the thickness of the surface roughness was calculated. The non-impingement surfaces of the airframe are thus subjected to the supersaturated air for extended periods and are likely to grow some ice layer or frost, which can degrade the performance and which have the potential to make the substrate for further ice growth by working in concert with other mechanisms.

As far as the interaction between roughness induced vortices and the droplets, it was shown that the unsteady vortex field can severely impact the paths followed by the droplets and cause impingements beyond the direct impingement region. However, only a certain range of droplets can end up with an impingement with the airframe surface. This is due to their relative inertia; they must have low enough inertia to be able to get into interaction with the vortices, and high enough inertia that helps them penetrate through the high momentum regions of a shed vortex and end up with an impingement. This

interaction results in impingements on areas that are beyond the direct impingement limits. For a particular case, up to $6.5 \%$ increase in the number of impingements was determined from the particle tracing in the unsteady flow. For the same particular case, the extra impingements led to $0.24 \mathrm{~mm}$ thick extra water accumulation in the non-impingement areas. 


\section{CHAPTER VII}

\section{RECOMMENDATIONS}

The droplet tracing in this study was done for a 2D flow over a NACA 0012 airfoil with glaze ice. However, it is obvious that a true picture of the flow physics over such geometry involves several 3D aspects, such as other two components of the vortices, and vortex stretching. In addition, to enhance the statistical accuracy, the considered period of unsteady flow can be extended. It is also desirable to see how these induced vortices interact with the surface water that forms due to the droplets that impinge on the airframe as a result of their interaction with the induced vortices. Considering a wide range of Reynolds numbers and roughness features will help determine the significance of this mechanisms in terms of ice accretion at different speeds, hence shed-vortex strength. In addition, there is a need for experimental verification of this mechanism by utilizing high-speed video camera recordings of the ice accretion experiments with a focus on the aft region of the main ice shape. These will help better understand the behavior of the droplets in this region and the motion of the water on the airfoil surface. Also, a fully 3D unsteady simulation along with the required experiments can help understand how the rime feathers and other roughness features beyond the glaze horn form.

As far as the frost growth, there is need for both theoretical and experimental studies. The existing frost growth models are valid for low speed flows over plain surfaces. An actual aircraft wing at the flight speed will require improvements on the existing frost growth models. Experimental support and evidence is also required to understand the contribution of frost to aircraft icing. 


\section{REFERENCES}

1. Bragg, M.B., Khodadoust, A., and Spring, S.A., "Measurements in a Leading-Edge Separation Bubble due to a Simulated Airfoil Ice Accretion,” AIAA Journal, Vol. 30, No. 6, pp.1462-1467, June 1992.

2. Messinger, B.L., "Equilibrium Temperature of an Unheated Icing Surface as a Function of Airspeed," Journal of the Aeronautical Sciences, Vol. 20, No. 1, pp. 29-42, 1953.

3. Broeren, A., Addy, H. E. Jr., and Bragg, M. B., "Effect of intercycle ice accretions on airfoil performance," AIAA-2002-240, AIAA Aerospace Sciences Meeting and Exhibit, 40th, Reno, NV, Jan. 14-17, 2002.

4. Whalen, E., Broeren, A., Bragg, M., and Lee, S., "Characteristics of Runback Ice Accretions on Airfoils and their Aerodynamics Effects," AIAA-2005-1065, AIAA Aerospace Sciences Meeting and Exhibit, 43 ${ }^{\text {rd }}$, Reno, Nevada, Jan. 10-13, 2005.

5. Cole, J.A., and Sand, W.R., "Statistical study of aircraft icing accidents," AIAA-1991-558, Aerospace Sciences Meeting, 29th, Reno, NV, Jan. 7-10, 1991.

6. Irvine, T.B., Kevdzija, S.L., Sheldon, D.W., and Spera, D.A., "Overview of the icing and flow quality improvements program for the NASA-Glenn Icing Research Tunnel," AIAA-2001-229, Aerospace Sciences Meeting and Exhibit, 39th, Reno, NV, Jan. 8-11, 2001.

7. Wright, B.W., "User Manual for the NASA Glenn Ice Accretion Code LEWICE,” NASA C.R.-1999209409, Brook Park, OH, Sept. 1999.

8. Morency, F., Beaugendre, H., Baruzzi, G.S., and Habashi, W.G., "FENSAP-ICE - A comprehensive 3D simulation system for in-flight icing," AIAA-2001-2566, AIAA Computational Fluid Dynamics Conference, 15th, Anaheim, CA, June 11-14, 2001.

9. http://www.airlinesafety.com/

10. Olsen, W. and Walker, E., "Experimental Evidence for Modifying the Current Physical Model for Ice Accretion on Aircraft Surfaces,” NASA-TM-87184, 1986.

11. Potapczuk, M.G., “A Review of NASA Lewis’ Development Plans for Computational Simulation of Aircraft Icing,” AIAA paper 99-0243, January 1999.

12. Rothmayer, A.P.and Krakos, J.A. "Residual droplet impacts and thin film dynamics," AIAA $42^{\text {nd }}$ Aerospace Sciences Meeting and Exhibit, 2004-0057, Reno, NV, January 2004.

13. NASA Lewis Research Center, "Validation report for LEWICE 2.0," NASA CR 208690 [CD-ROM], January 1999.

14. Huebsch, W.W. and Rothmayer, A.P., "Effects of surface ice roughness on dynamic stall," Journal of Aircraft, Vol. 39, No. 6, pp 945-953, 2002. 
15. Lee, S., Dunn, T., Gurbacki, H.M., Bragg, M.B., and Loth, E., “An Experimental and Computational Investigation of Spanwise-Step-Ice Shapes on Airfoil Aerodynamics" AIAA paper 98-0490, Aerospace Sciences Meeting and Exhibit, 36 ${ }^{\text {th }}$, Reno, NV, January 1998.

16. Dunn, T.A. and Loth, E., "Effects of Simulated-Spanwise-Ice Shapes on Airfoils: Computational Investigation" AIAA paper 99:-0093, Aerospace Sciences Meeting and Exhibit, 37 $7^{\text {th }}$, Reno, NV, January 1999.

17. Chung, J.J. and Addy, H.E. Jr., “A Numerical Evaluation of Icing Effects on a Natural Laminar Flow Airfoil" AIAA paper 2000-0096, Aerospace Sciences Meeting and Exhibit, 38 ${ }^{\text {th }}$, Reno, NV, January 2000.

18. Pan, J., Loth, E. and Bragg, M., "RANS Simulations of Airfoils with Ice Shapes" AIAA paper 2003729, Aerospace Sciences Meeting and Exhibit, 41 ${ }^{\text {st }}$, Reno, NV, January 2003.

19. Ogretim, E. and Huebsch, W., "A Novel Method for Automated Grid Generation of Ice Shapes for Local-Flow Analysis" International Journal for Numerical Methods in Fluids, Volume 44, pp. 579$597,2004$.

20. Bhaskaran, R., "Unsteady Incompressible Viscous Flow Past Stationary, Pitching or Oscillating Airfoil Leading-Edges” dissertation study, Iowa State University, Ames, Iowa, 1996.

21. Davis, R.T., Ghia, U., and Ghia, K.H., "Laminar Incompressible Flow past a Class of Blunted Wedges Using the Navier-Stokes Equations” Computers and Fluids, Volume 2, pp. 211-223, 1974.

22. Beierle, M.T., Anderson, Jr. J.D., and Ranzenbach, R.C., "Computational Investigation of Roughness Related Lift Enhancement to Symmetric Airfoils" AIAA paper 1999-3135, AIAA Applied Aerodynamics Conference, 17th, Norfolk, VA, June 28-July 1, 1999.

23. Raghunathan, S., Harrison, J. R., and Hawkins, B. D., "Thick Airfoil at low Reynolds Number and High Incidence" Journal of Aircraft, Vol. 25, No. 7, pp. 669-671, 1988.

24. Bragg, M.B. and Khodadoust, A., "Experimental Measurements in a Large Separation Bubble Due to a Simulated Glaze Ice Accretion," AIAA paper 88-0116, Aerospace Sciences Meeting, 26th, Reno, NV, Jan. 11-14, January 1988.

25. Gurbacki, H.M. and Bragg, M.B., "Unsteady Aerodynamic Measurements on an Iced Airfoil," AIAA paper 2002-0241, January, 2002.

26. Bencic, T.J., “Application of Pressure-Sensitive Paint to Ice-Accreted Wind Tunnel Models," AIAA paper 2000-0834, January 2000.

27. Ferrigno, F., Guille, M., Auletta, A. and Palazzo, S., "Low speed PSP Measurements on an Ice Accretion Shaped Model," AIAA paper 2003-744, January 2003.

28. Ogretim, E., Huebsch, W., and Narramore, J., "The effects of leading-edge ice roughness on downstream frost formation," AIAA paper 2004-0059, January 2004. 
29. Emanuel, K.A., "Moist Thermodynamic Processes," Atmospheric Convection, Oxford University Press, New York, 1994, pp. 107-117.

30. Rogers, R.R., A Short Course in Cloud Physics, $2^{\text {nd }}$ ed., Pergamon Press, New York, 1979, pp. 74-75, 55.

31. Santos, L.C.C., Neto, L.T., Papa, R., Oliveira, G.L., Jesus, A.B., and Wirogo, S., "Grid sensitivity effects in collection efficiency computation," AIAA paper 2004-0566, 42 ${ }^{\text {nd }}$ Aerospace Sciences Meeting \& Exhibit, Reno, NV, January 2004.

32. Tan, C, "A tentative mass loss model for simulating water droplet splash," AIAA paper 2004-0410, $42^{\text {nd }}$ Aerospace Sciences Meeting \& Exhibit, Reno, NV, January 2004.

33. Tan, S.C. and Papadakis, M., "General effects of large droplet dynamics on ice accretion modeling," AIAA paper 2003-0392, $41^{\text {st }}$ Aerospace Sciences Meeting \& Exhibit, Reno, NV, January 2003.

34. Rutkowski, A., Wright, W.B., and Potapczuk, M.G., "Numerical study of droplet splashing and reimpingement," AIAA paper 2003-0388, 41 ${ }^{\text {st }}$ Aerospace Sciences Meeting \& Exhibit, Reno, NV, January 2003.

35. Reehorst, A.L., Ratvasky, T.P., and Sims, J., "Close-up analysis of inflight ice accretion,” NASA TM-106457, January 2004.

36. White, F.M., Viscous Fluid Flow. McGraw-Hill, Inc, $2^{\text {nd }}$ edition, pp. 182.

37. Bragg, M.B., "Rime Ice Accretion and Its Effect on Airfoil Performance," NACA CR-165599, March 1982.

38. Dompierre, J., Cronin, D.J., Bourgault, Y., Baruzzi, G., Habashi, W.G., and Wagner, G.A., "Numerical Simulation of Performance Degradation of Ice Contaminated Airfoils," AIAA paper 1997-2235, $15^{\text {th }}$ AIAA Applied Aerodynamics Conference, Atlanta, GA, June, 1997.

39. Storey, B.D. and Jacobi, A.M., "The effect of streamwise vortices on the frost growth rate in developing laminar channel flows," International Journal of Heat and Mass Transfer, Volume 42, No. 20, 1999, pp. 3787-3802.

40. Ismail, K.A.R., Salinas, C. And Gonçalves, M.M., "Frost growth around a cylinder in a wet air stream”, International Journal of Refrigeration, Vol. 20, No. 2, pp. 106-119, 1997.

41. Tao, Y.-X., Besant, R.W. and Rezkallah, K.S., "A mathematical model for predicting the densification and growth of frost on a flat plate", International Journal of Heat and Mass Transfer, Vol. 36, No. 2, pp. 353-363, 1993.

42. Schneider, H.W., "Equation of the growth rate of frost forming on cooled surfaces", International Journal of Heat and Mass Transfer, Vol. 21, pp. 1019-1024, 1978.

43. Na, B. and Webb, R.L., "New model for frost growth rate", International Journal of Heat and Mass Transfer, Vol. 47, pp. 925-936, 2004. 
44. Na, B. and Webb, R.L., "Mass transfer on and within a Frost Layer", International Journal of Heat and Mass Transfer, Vol. 47, pp. 899-911, 2004.

45. Yamaguchi, K. and Hansman, R. J., JR., "Heat transfer on accreting ice surfaces", Journal of Aircraft, Vol. 29, no.1, pp. 108-113, 1992. 


\section{APPENDICES}

\section{APPENDIX A: AIRFOIL SHAPE}

\section{Airfoil Coordinates}

In the non-dimensional form, all NACA 00XX airfoils' coordinates are given by the formula below.

$$
\frac{y}{c}=\frac{t / c}{0.20}\left[0.2969 \sqrt{(x / c)}-0.1260(x / c)-0.3516(x / c)^{2}+0.2843(x / c)^{3}-0.1015(x / c)^{4}\right]
$$

where $t / c$ is the thickness ratio of the relevant airfoil (NASA-TM-4741). Due to the nature of the formulations in this study, the coordinates need to be non-dimensionalized by the leading-edge radius of the airfoil. Also, a shift in the $x$-coordinates is necessary to approach the parabola leading-edge and express the airfoil geometry and the ice roughness on it as perturbations on the parabola leading-edge. So the final non-dimensionalization for the $x$ and $y$ coordinates become:

$$
x^{\prime}=\frac{x}{l e r}=\frac{x / c}{l e r / c}-\frac{1}{2}, y^{\prime}=\frac{y}{l e r}=\frac{y / c}{l e r / c}
$$

where $l e r / c$ is the non-dimensional leading-edge radius of the airfoil and $\mathrm{d}$ is the denominator of the transformation for $x$ as explained below.

The airfoil coordinates are transformed to the parabolic coordinates following this nondimensionalization. This transformation is done by using the following formulae:

$$
x^{\prime}=\frac{\xi^{2}-\eta^{2}}{2}, y^{\prime}=\xi^{\prime} \eta^{\prime}
$$

Using the Prandtl transposition to separate the perturbations from the base geometry and embed them into the governing equations, the transformation to parabolic coordinates can be updated as follows:

$$
\begin{gathered}
\xi=\xi^{\prime}, \eta=\eta^{\prime}-f \\
x^{\prime}=\frac{\xi^{2}-(\eta+f)^{2}}{2}, y^{\prime}=\xi(\eta+f)
\end{gathered}
$$

where $f$ is a single-valued function describing the perturbation geometry as a function of $\xi$. Based on these, the non-dimensional $\mathrm{x}-\mathrm{y}$ values based on the chord length can be written as: 


$$
\frac{x}{c}=\left[\frac{\xi^{2}-(\eta+f)^{2}}{2}+\frac{\eta_{d}^{2}}{2}\right](l e r / c), \frac{y}{c}=\xi(\eta+f)(\text { ler } / c)
$$

where $\eta_{d}$ is the $\eta$ value at the surface of the base parabola. The $f$ values are the perturbations for the airfoil coordinates from the base parabola surface. Therefore, the $\eta$ value for the airfoil coordinates is $\eta_{d}$. So, inserting these into the above transformation functions, we can update the airfoil coordinate equation and get an equation for the $f$ values in terms of $\xi$.

$$
\begin{gathered}
\frac{x}{c}=\left[\xi^{2}-2 f-f^{2}\right] \frac{(\text { ler } / c)}{2}, \frac{y}{c}=\xi(1+f)(\text { ler } / c) \\
f=\frac{t / c}{0.20(\text { ler } / c) \xi}\left[0.2969 \sqrt{\beta}-0.1260 \beta-0.3516 \beta^{2}+0.2843 \beta^{3}-0.1015 \beta^{4}\right]-\eta_{d}, \text { where } \\
\beta=\frac{(\text { ler } / c)}{2}\left[\xi^{2}-2 f-f^{2}\right] .
\end{gathered}
$$

Based on the above formula, the derivatives of the perturbation geometry (i.e. airfoil shape in the transformed plane) can also be obtained. For the purposes of the present study, the first and the second derivatives are of concern and they are given below.

$$
\begin{gathered}
f_{\xi}=\frac{(t / c)}{\left[0.20+\frac{\theta(t / c)(1+f)}{\xi}\right]}\left(\theta-\frac{\phi}{(\text { ler } / c) \xi^{2}}\right) \\
f_{\xi \xi}=-\left[\frac{\xi(t / c)}{0.20(\text { term } 1)}\right]^{2}(\text { term } 2)\left[\theta-\frac{\phi}{(\text { ler } / c) \xi^{2}}\right]+(\text { term } 3)
\end{gathered}
$$

where, $\phi=0.2969 \sqrt{\beta}-0.1260 \beta-0.3516 \beta^{2}+0.2843 \beta^{3}-0.1015 \beta^{4}$

$$
\begin{gathered}
\theta=\phi_{\beta}=\frac{0.2969}{2 \sqrt{\beta}}-0.1260-0.7032 \beta+0.8529 \beta^{2}-0.4060 \beta^{3} \\
\operatorname{term} 1=\xi+\frac{\theta(t / c)(1+f)}{0.20} \\
\operatorname{term} 2=\frac{\left[\mu \beta_{\xi}(1+f)+f_{\xi} \theta\right]}{\xi}-\frac{\theta(1+f)}{\xi^{2}}
\end{gathered}
$$




$$
\begin{gathered}
\text { term } 3=\frac{\xi(t / c)}{0.20 \text { term } 1}\left(-\frac{\theta \beta_{\xi}}{(\text { ler } / c) \xi^{2}}+\frac{2 \xi \phi}{(\text { ler } / c) \xi^{4}}+\mu \beta_{\xi}\right) \\
\mu=\phi_{\beta \beta}=\theta_{\beta}=-\frac{0.2969}{4 \beta^{1.5}}-0.7032+1.7058 \beta-1.218 \beta^{2} \\
\beta_{\xi}=(\text { ler } / c)\left[\xi-f_{\xi}(1+f)\right]
\end{gathered}
$$

The equation for $f$ is an implicit one which requires an iterative method to solve for. For the iterations, the Newton method is used. This method requires the first derivative of the function, which is given as:

\section{The Newton Method}

The Newton method is an easy-to-apply iterative method that has first order accuracy. Let $g$ be a

function of $f$. We are looking for the zeros of the function $g$ near a given point, which is the first guess for the iterations. Then the recursion formula is:

$$
f_{n+1}=f_{n}-\frac{g\left(f_{n}\right)}{g^{\prime}\left(f_{n}\right)}, \text { where } g^{\prime}\left(f_{n}\right) \text { is the derivative of } g \text { with respect to } f \text { at the most recent value of } f \text {. }
$$

For the present study:

$$
\begin{gathered}
g=\frac{t / c}{0.20(\operatorname{ler} / c) \xi}\left[0.2969 \sqrt{\beta}-0.1260 \beta-0.3516 \beta^{2}+0.2843 \beta^{3}-0.1015 \beta^{4}\right]-\eta_{d}-f \\
g^{\prime}=\frac{-(t / c)(1+f)}{0.20 \xi} \theta-1
\end{gathered}
$$




\section{APPENDIX B: DIFFERENTIAL GEOMETRY}

\section{Unit vectors}

$$
\begin{gathered}
x=\frac{\xi^{\prime 2}-\eta^{\prime 2}}{2} \quad y=\xi^{\prime} \eta^{\prime} \\
h_{1}=\sqrt{x_{\xi^{\prime}}^{2}+y_{\xi^{\prime}}^{2}} \quad h_{1}=\sqrt{\xi^{\prime 2}+\eta^{\prime 2}} \\
h_{2}=\sqrt{x_{\eta^{\prime}}^{2}+y_{\eta^{\prime}}^{2}} \quad h_{2}=\sqrt{\left(-\eta^{\prime}\right)^{2}+\xi^{\prime 2}} \\
h=h_{1}=h_{2}=\sqrt{\xi^{\prime 2}+\eta^{\prime 2}}
\end{gathered}
$$

Let $\vec{r}$ be the position vector. Then:

$$
\begin{gathered}
\vec{r}=x \hat{i}+y \hat{j} \\
\vec{r}_{\xi^{\prime}}=h_{1} \hat{e}_{\xi^{\prime}} \text { and } \vec{r}_{\eta^{\prime}}=h_{2} \hat{e}_{\eta^{\prime}}
\end{gathered}
$$

From taking the dot product of each reference unit vector with itself, we begin the derivation.

$$
\hat{e}_{\xi^{\prime}} \cdot \hat{e}_{\xi^{\prime}}=1 \text { and } \hat{e}_{\eta^{\prime}} \cdot \hat{e}_{\eta^{\prime}}=1
$$

We can differentiate these equations with respect to either $\xi^{\prime}$ or $\eta^{\prime}$.

$$
2 \hat{e}_{\xi^{\prime}} \cdot \frac{\partial \hat{e}_{\xi^{\prime}}}{\partial \xi^{\prime}}=0 \text { and } 2 \hat{e}_{\eta^{\prime}} \cdot \frac{\partial \hat{e}_{\eta^{\prime}}}{\partial \eta^{\prime}}=0
$$

Let's work on one of them; the other cases can be worked out accordingly. For the first equation, we know that the $\xi^{\prime}$ reference unit vector is purely in $\xi^{\prime}$ direction. In order to have the dot product as zero, the derivative term must be purely in $\eta^{\prime}$ direction. Mathematically speaking:

$$
\begin{gathered}
\frac{\partial \hat{e}_{\xi^{\prime}}}{\partial \xi^{\prime}}=A_{1} \hat{e}_{\xi^{\prime}}+A_{2} \hat{e}_{\eta^{\prime}} \\
2 \hat{e}_{\xi^{\prime}} \cdot \frac{\partial \hat{e}_{\xi^{\prime}}}{\partial \xi^{\prime}}=0, \text { so } 2 \hat{e}_{\xi^{\prime}} \cdot\left(A_{1} \hat{e}_{\xi^{\prime}}+A_{2} \hat{e}_{\eta^{\prime}}\right)=0 \text { then } A_{1}=0
\end{gathered}
$$




$$
\begin{aligned}
& \vec{r}_{\xi^{\prime} \xi^{\prime}}=h_{1_{\xi^{\prime}}} \hat{e}_{\xi^{\prime}}+h_{1} \frac{\partial \hat{e}_{\xi^{\prime}}}{\partial \xi^{\prime}}=h_{1_{\xi^{\prime}}} \hat{e}_{\xi^{\prime}}+h_{1} A_{2} \hat{e}_{\eta^{\prime}} \\
& \vec{r}_{\xi^{\prime} \xi^{\prime}} \cdot \hat{e}_{\eta^{\prime}}=h_{1} A_{2}
\end{aligned}
$$

Then

$$
\begin{gathered}
A_{2}=\frac{1}{h_{1}}\left(\vec{r}_{\xi^{\prime} \xi^{\prime}}, \hat{e}_{\eta^{\prime}}\right)=\frac{1}{h_{1} h_{2}}\left(\vec{r}_{\xi^{\prime} \xi^{\prime}} \cdot \vec{r}_{\eta^{\prime}}\right) \text { hence } \frac{\partial \hat{e}_{\xi^{\prime}}}{\partial \xi^{\prime}}=\frac{1}{h_{1} h_{2}}\left(\vec{r}_{\xi^{\prime} \xi^{\prime}}, \vec{r}_{\eta^{\prime}}\right) \hat{e}_{\eta^{\prime}} \\
\vec{r}_{\xi^{\prime} \xi^{\prime}}=x_{\xi^{\prime} \xi^{\prime}} \hat{i}+y_{\xi^{\prime} \xi^{\prime}} \hat{j}=\hat{i} \text { and } \vec{r}_{\eta^{\prime}}=x_{\eta^{\prime}} \hat{i}+y_{\eta^{\prime}} \hat{j}=-\eta \hat{i}+\hat{j}
\end{gathered}
$$

Finally the derivatives of the unit vectors along the coordinate axes are determined to be:

$$
\begin{array}{ll}
\frac{\partial \hat{e}_{\xi^{\prime}}}{\partial \xi^{\prime}}=-\left(\frac{\eta^{\prime}}{\xi^{\prime 2}+\eta^{\prime 2}}\right) \hat{e}_{\eta^{\prime}} & \frac{\partial \hat{e}_{\eta^{\prime}}}{\partial \xi^{\prime}}=\left(\frac{\eta^{\prime}}{\xi^{\prime 2}+\eta^{\prime 2}}\right) \hat{e}_{\xi^{\prime}} \\
\frac{\partial \hat{e}_{\xi^{\prime}}}{\partial \eta^{\prime}}=\left(\frac{\xi^{\prime}}{\xi^{\prime 2}+\eta^{\prime 2}}\right) \hat{e}_{\eta^{\prime}} & \frac{\partial \hat{e}_{\eta^{\prime}}}{\partial \eta^{\prime}}=-\left(\frac{\xi^{\prime}}{\xi^{\prime 2}+\eta^{\prime 2}}\right) \hat{e}_{\xi^{\prime}}
\end{array}
$$

\section{Gradient operator}

Apply the transformation by using the following formula

$$
\nabla=\frac{\hat{e}_{\xi^{\prime}}}{h_{1}} \frac{\partial}{\partial \xi^{\prime}}+\frac{\hat{e}_{\eta^{\prime}}}{h_{2}} \frac{\partial}{\partial \eta^{\prime}}
$$

Gradient of a scalar function in the new coordinate system

$$
\nabla \phi=\frac{\hat{e}_{\xi^{\prime}}}{h_{1}} \phi_{\xi^{\prime}}+\frac{\hat{e}_{\eta^{\prime}}}{h_{2}} \phi_{\eta^{\prime}} \text { hence } \nabla \phi=\frac{1}{\sqrt{\xi^{\prime 2}+\eta^{\prime 2}}}\left(\phi_{\xi^{\prime}} \hat{e}_{\xi^{\prime}}+\phi_{\eta^{\prime}} \hat{e}_{\eta^{\prime}}\right)
$$

\section{Divergence operator}

Divergence of a vectorial function in the new coordinate system

$$
\begin{gathered}
\nabla \cdot \vec{A}=\frac{1}{h_{1} h_{2}}\left[\left(h_{2} A_{1}\right)_{\xi^{\prime}}+\left(h_{1} A_{2}\right)_{\eta^{\prime}}\right] \text { where } \vec{A}=A_{1} \hat{e}_{\xi^{\prime}}+A_{2} \hat{e}_{\eta^{\prime}} \\
\text { hence } \nabla \cdot \vec{A}=\frac{1}{\xi^{\prime 2}+\eta^{\prime 2}}\left[\left(\left(\sqrt{\xi^{\prime 2}+\eta^{\prime 2}}\right) A_{1}\right)_{\xi^{\prime}}+\left(\left(\sqrt{\xi^{\prime 2}+\eta^{\prime 2}}\right) A_{2}\right)_{\eta^{\prime}}\right]
\end{gathered}
$$




\section{Laplacian operator}

$$
\nabla \cdot \vec{A}=\frac{1}{\xi^{\prime 2}+\eta^{\prime 2}}\left[\left(\left(\sqrt{\xi^{\prime 2}+\eta^{\prime 2}}\right) A_{1}\right)_{\xi^{\prime}}+\left(\left(\sqrt{\xi^{\prime 2}+\eta^{\prime 2}}\right) A_{2}\right)_{\eta^{\prime}}\right]
$$

From this:

$$
\nabla . \nabla=\frac{1}{h^{2}}\left(\frac{\partial^{2}}{\partial \xi^{\prime 2}}+\frac{\partial^{2}}{\partial \eta^{\prime 2}}\right)
$$

Laplacian of a scalar function in the new coordinate system

$$
\nabla^{2} \phi=\frac{1}{h_{1} h_{2}}\left[\left(\frac{h_{2}}{h_{1}} \phi_{\xi^{\prime}}\right)_{\xi^{\prime}}+\left(\frac{h_{1}}{h_{2}} \phi_{\eta^{\prime}}\right)_{\eta^{\prime}}\right] \text { hence } \nabla^{2} \phi=\frac{1}{\xi^{\prime 2}+\eta^{\prime 2}}\left[\phi_{\xi^{\prime} \xi^{\prime}}+\phi_{\eta^{\prime} \eta^{\prime}}\right]
$$

and Laplacian of a vectorial function $\vec{A}$ becomes:

$$
\begin{aligned}
& \nabla^{2} \vec{A}=\frac{1}{h^{2}}\left(\frac{\partial}{\partial \xi^{\prime 2}}+\frac{\partial}{\partial \eta^{\prime 2}}\right)\left(A_{1} \hat{e}_{\xi^{\prime}}+A_{2} \hat{e}_{\eta^{\prime}}\right)
\end{aligned}
$$

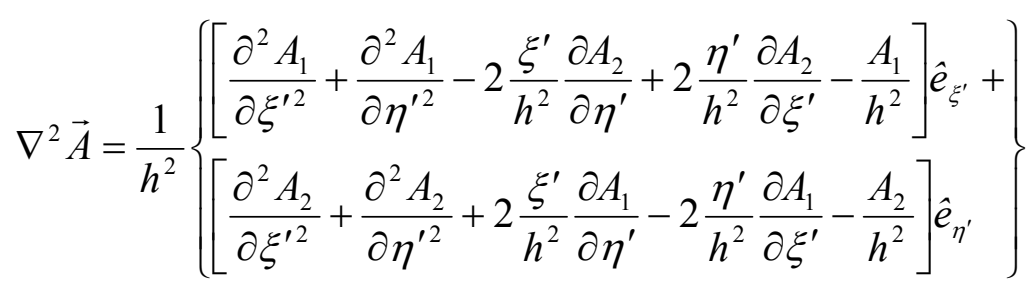

\section{Prandtl transposition}

Prandtl transposition (shearing transformation) is applied in order to suppress the perturbations on the parabola surface. As a result of this, the perturbation geometry is embedded in the wall boundary condition equations. The chain rules that are used for the application of the Prandtl transposition are:

$$
\begin{aligned}
\frac{\partial}{\partial \xi^{\prime}} & =\frac{\partial}{\partial \xi}-f_{\xi} \frac{\partial}{\partial \eta} & \frac{\partial^{2}}{\partial \xi^{\prime 2}} & =\frac{\partial^{2}}{\partial \xi^{2}}-f_{\xi \xi} \frac{\partial}{\partial \eta}-2 f_{\xi} \frac{\partial^{2}}{\partial \xi \partial \eta}+f_{\xi}^{2} \frac{\partial^{2}}{\partial \eta^{2}} \\
\frac{\partial}{\partial \eta^{\prime}} & =\frac{\partial}{\partial \eta} & \frac{\partial^{2}}{\partial \eta^{\prime 2}} & =\frac{\partial^{2}}{\partial \eta^{2}}
\end{aligned}
$$




\section{Fourier expansion}

For the cases in which complex roughness shapes are involved (i.e. ice roughness) the Fourier analysis is used to obtain the analytical expression of the roughness geometry. The Fourier analysis requires the original roughness geometry data as well as the transformed version into the $\xi^{\prime}-\eta^{\prime}$ plane. After this is completed, the following equations are used in order to obtain the analytical expression for the roughness shape:

$$
\begin{aligned}
& a_{i}=\frac{2}{\left(\xi_{N}-\xi_{1}\right)} \int_{\xi_{1}}^{\xi_{N}} f(\xi) * \cos \left(\frac{2 \pi i \xi}{\left(\xi_{N}-\xi_{1}\right)}\right) d \xi \mathrm{i}=0,1,2, \ldots \\
& b_{i}=\frac{2}{\left(\xi_{N}-\xi_{1}\right)} \int_{\xi_{1}}^{\xi_{N}} f(\xi) * \sin \left(\frac{2 \pi i \xi}{\left(\xi_{N}-\xi_{1}\right)}\right) d \xi \mathrm{i}=1,2,3, \ldots
\end{aligned}
$$

Then,

$$
\tilde{f}(\xi)=\frac{1}{2} a_{0}+\sum_{i=1}^{i=N}\left[\begin{array}{l}
a_{i} \cos \left(\frac{2 \pi i \xi}{\left(\xi_{N}-\xi_{1}\right)}\right) \\
+b_{i} \sin \left(\frac{2 \pi i \xi}{\left(\xi_{N}-\xi_{1}\right)}\right)
\end{array}\right]
$$

where $\xi_{1}$ and $\xi_{\mathrm{N}}$ are the first and the last points, respectively, $\mathrm{a}_{\mathrm{i}}$ 's are the coefficients of the cosine terms, $\mathrm{b}_{\mathrm{i}}$ 's are the coefficients of the sine terms, $f(\xi)$ is the transformed roughness data and $\widetilde{f}(\xi)$ is the analytic roughness expression in terms of the sine and cosine functions in the $\xi-\eta$ plane. 


\section{APPENDIX C: DERIVATION OF THE PRESSURE POISSON EQUATION}

\section{Laplacian of the pressure}

Using the gradient operator as defined before, the closed form of the pressure Poisson equation can be expanded as follows:

$$
\begin{gathered}
\nabla^{2} C_{p}=-2 \nabla \cdot[(\vec{V} \cdot \nabla) \vec{V}] \\
C_{p_{\xi^{\prime} \xi^{\prime}}}+C_{p_{\eta^{\prime} \eta^{\prime}}}=R \text { where } R=-2 \nabla \cdot[(\vec{V} \cdot \nabla) \vec{V}] .
\end{gathered}
$$

Then, using the Prandtl transposition, the surface perturbation geometry can be embedded into the pressure Poisson equation. This introduces terms related to the surface derivatives. However, the character equation still remains the same; it is still an elliptic equation.

$$
C_{p_{\xi \xi}}+\left(1+f_{\xi}^{2}\right) C_{p_{\eta \eta}}-f_{\xi \xi} C_{p_{\eta}}-2 f_{\xi} C_{p_{\xi \eta}}=R
$$

\section{Right hand side of the Poisson equation}

$$
\begin{gathered}
\nabla^{2} C_{p}=-2 \nabla \cdot[(\vec{V} \cdot \nabla) \vec{V}] \\
\vec{V} . \nabla=\frac{u}{\sqrt{\xi^{\prime 2}+\eta^{\prime 2}}} \frac{\partial}{\partial \xi^{\prime}}+\frac{v}{\sqrt{\xi^{\prime 2}+\eta^{\prime 2}}} \frac{\partial}{\partial \eta^{\prime}} \\
(\vec{V} . \nabla) \vec{V}=\frac{1}{\sqrt{\xi^{\prime 2}+\eta^{\prime 2}}}\left\{\left[u u_{\xi^{\prime}}+\frac{u v \eta^{\prime}}{\xi^{\prime 2}+\eta^{\prime 2}}+v u_{\eta^{\prime}}-\frac{v^{2} \xi^{\prime}}{\xi^{\prime 2}+\eta^{\prime 2}}\right] \hat{e}_{\xi^{\prime}}+\left[u v_{\xi^{\prime}}-\frac{u^{2} \eta^{\prime}}{\xi^{\prime 2}+\eta^{\prime 2}}+v v_{\eta^{\prime}}+\frac{v u \xi^{\prime}}{\xi^{\prime 2}+\eta^{\prime 2}}\right] \hat{e}_{\eta^{\prime}}\right\} \\
\nabla .[(\vec{V} . \nabla) \vec{V}]=\frac{1}{\xi^{\prime 2}+\eta^{\prime 2}}\left\{\frac{\partial}{\partial \xi^{\prime}}\left[u u_{\xi^{\prime}}+v u_{\eta^{\prime}}+\frac{\left(u \eta^{\prime}-v \xi^{\prime}\right) v}{\xi^{\prime 2}+\eta^{\prime 2}}\right]+\frac{\partial}{\partial \eta^{\prime}}\left[u v_{\xi^{\prime}}+v v_{\eta^{\prime}}+\frac{\left(v \xi^{\prime}-u \eta^{\prime}\right) u}{\xi^{\prime 2}+\eta^{\prime 2}}\right]\right\}
\end{gathered}
$$

By regrouping the terms inside the square parenthesis and using the continuity equation

$$
\nabla .[(\vec{V} . \nabla) \vec{V}]=\frac{1}{\xi^{\prime 2}+\eta^{\prime 2}}\left\{\begin{array}{l}
\frac{\partial}{\partial \xi^{\prime}}\left[-u\left(v_{\eta^{\prime}}+\frac{u \xi^{\prime}}{\xi^{\prime 2}+\eta^{\prime 2}}\right)+v\left(u_{\xi^{\prime}}-\frac{v \xi^{\prime}}{\xi^{\prime 2}+\eta^{\prime 2}}\right)\right]+ \\
\frac{\partial}{\partial \eta^{\prime}}\left[u\left(v_{\xi^{\prime}}-\frac{u \eta^{\prime}}{\xi^{\prime 2}+\eta^{\prime 2}}\right)-v\left(u_{\xi^{\prime}}+\frac{v \eta^{\prime}}{\xi^{\prime 2}+\eta^{\prime 2}}\right)\right]
\end{array}\right\}
$$


After taking all the derivatives, it is seen that the double (or cross) derivatives and also some other terms cancel each other. And then by regrouping the parenthesis, the following are obtained,

$$
\nabla .[(\vec{V} . \nabla) \vec{V}]=\frac{-2}{\xi^{\prime 2}+\eta^{\prime 2}}\left[u_{\xi^{\prime}}\left(v_{\xi^{\prime}}+\frac{u \xi^{\prime}}{\xi^{\prime 2}+\eta^{\prime 2}}\right)-v_{\xi^{\prime}}\left(u_{\xi^{\prime}}-\frac{v \xi^{\prime}}{\xi^{\prime 2}+\eta^{\prime 2}}\right)+\frac{u u_{\eta^{\prime}} \eta^{\prime}+v v_{\eta^{\prime}} \eta^{\prime}}{\xi^{\prime 2}+\eta^{\prime 2}}\right]
$$

split the last term and add and subtract $\frac{u v \xi^{\prime} \eta^{\prime}}{\left(\xi^{\prime 2}+\eta^{\prime 2}\right)^{2}}$ and then by regrouping

$$
\begin{gathered}
\nabla .[(\vec{V} . \nabla) \vec{V}]=\frac{-2}{\xi^{\prime 2}+\eta^{\prime 2}}\left[\left(u_{\xi^{\prime}}+\frac{v \eta^{\prime}}{\xi^{\prime 2}+\eta^{\prime 2}}\right)\left(v_{\eta^{\prime}}+\frac{u \xi^{\prime}}{\xi^{\prime 2}+\eta^{\prime 2}}\right)-\left(v_{\xi^{\prime}}-\frac{u \eta^{\prime}}{\xi^{\prime 2}+\eta^{\prime 2}}\right)\left(u_{\eta^{\prime}}-\frac{v \xi^{\prime}}{\xi^{\prime 2}+\eta^{\prime 2}}\right)\right] \\
R=4\left[\left(u_{\xi^{\prime}}+\frac{v \eta^{\prime}}{\xi^{\prime 2}+\eta^{\prime 2}}\right)\left(v_{\eta^{\prime}}+\frac{u \xi^{\prime}}{\xi^{\prime 2}+\eta^{\prime 2}}\right)-\left(v_{\xi^{\prime}}-\frac{u \eta^{\prime}}{\xi^{\prime 2}+\eta^{\prime 2}}\right)\left(u_{\eta^{\prime}}-\frac{v \xi^{\prime}}{\xi^{\prime 2}+\eta^{\prime 2}}\right)\right]
\end{gathered}
$$

Then using the Prandtl transposition, the final version is obtained for the right-hand-side of the pressure Poisson equation.

$$
R=4\left[\begin{array}{l}
\left(u_{\xi}-f_{\xi} u_{\eta}+\frac{v(\eta+f)}{\xi^{2}+(\eta+f)^{2}}\right)\left(v_{\eta}+\frac{u \xi}{\xi^{2}+(\eta+f)^{2}}\right)- \\
\left(v_{\xi}-f_{\xi} v_{\eta}-\frac{u \eta}{\xi^{2}+(\eta+f)^{2}}\right)\left(u_{\eta}-\frac{v \xi}{\xi^{2}+(\eta+f)^{2}}\right)
\end{array}\right]
$$

\section{Wall boundary condition}

The derivation starts with the primitive variables version of the N-S equations, and then the no-slip BC is applied.

$$
\vec{V}_{t}+(\vec{V} \cdot \nabla) \vec{V}=-\nabla C_{p}+R e^{-1} \nabla^{2} \vec{V}
$$

But $\vec{V}=0$ at the wall, so,

$$
\nabla C_{p}=R e^{-1} \nabla^{2} \vec{V}, \text { where } \vec{V}=u \hat{e}_{\xi^{\prime}}+v \hat{e}_{\eta^{\prime}}
$$

The definitions for gradient and Laplacian in the transformed $\left(\xi^{\prime}-\eta^{\prime}\right)$ plane $\operatorname{are}^{20}$ : 


$$
\nabla \phi=\frac{1}{\sqrt{\xi^{\prime 2}+\eta^{\prime 2}}}\left(\phi_{\xi^{\prime}} \hat{e}_{\xi^{\prime}}+\phi_{\eta^{\prime}} \hat{e}_{\eta^{\prime}}\right), \text { hence } \nabla C_{p}=\frac{1}{h}\left(\frac{\partial C_{p}}{\partial \xi^{\prime}} \hat{e}_{\xi^{\prime}}+\frac{\partial C_{p}}{\partial \eta^{\prime}} \hat{e}_{\eta^{\prime}}\right)
$$

For the right-hand-side of the equation, the Laplacian of the velocity vector is required.

$$
\nabla^{2} \vec{A}=\frac{1}{h^{2}}\left(\frac{\partial}{\partial \xi^{\prime 2}}+\frac{\partial}{\partial \eta^{\prime 2}}\right)\left(A_{1} \hat{e}_{\xi^{\prime}}+A_{2} \hat{e}_{\eta^{\prime}}\right), \text { where } h=\sqrt{\xi^{\prime 2}+\eta^{\prime 2}}
$$

The Laplacian expression needs special attention when expanding. This is due to the fact that the normal vectors in the $\xi^{\prime}-\eta^{\prime}$ plane change as a function of the local coordinates. Taking this into account, the following is obtained:

$$
\nabla^{2} \vec{A}=\frac{1}{h^{2}}\left\{\begin{array}{l}
{\left[\frac{\partial^{2} A_{1}}{\partial \xi^{\prime 2}}+\frac{\partial^{2} A_{1}}{\partial \eta^{\prime 2}}-2 \frac{\xi^{\prime}}{h^{2}} \frac{\partial A_{2}}{\partial \eta^{\prime}}+2 \frac{\eta^{\prime}}{h^{2}} \frac{\partial A_{2}}{\partial \xi^{\prime}}-\frac{A_{1}}{h^{2}}\right] \hat{e}_{\xi^{\prime}}+} \\
{\left[\begin{array}{l}
\partial^{2} A_{2} \\
\partial \xi^{\prime 2}
\end{array}+\frac{\partial^{2} A_{2}}{\partial \eta^{\prime 2}}+2 \frac{\xi^{\prime}}{h^{2}} \frac{\partial A_{1}}{\partial \eta^{\prime}}-2 \frac{\eta^{\prime}}{h^{2}} \frac{\partial A_{1}}{\partial \xi^{\prime}}-\frac{A_{2}}{h^{2}}\right] \hat{e}_{\eta^{\prime}}}
\end{array}\right\}
$$

Then, the pressure equation at the wall boundary becomes,

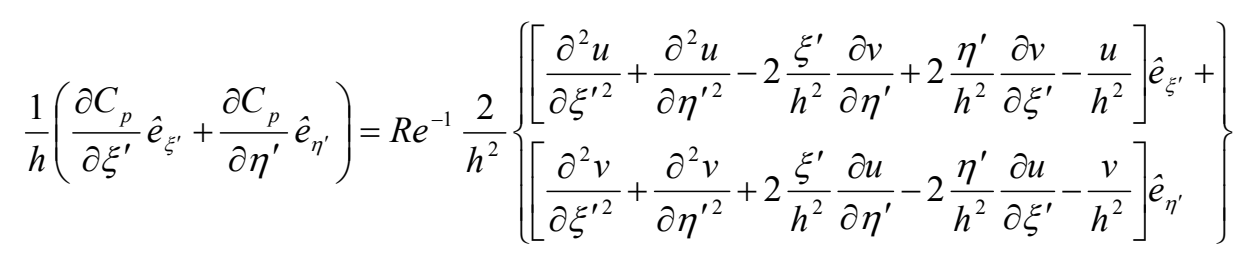

Using the no slip condition, and taking the dot product of both sides of the above equation with $\hat{e}_{\xi^{\prime}}$ and $\hat{e}_{\eta^{\prime}}$ one gets:

$$
\begin{aligned}
& \frac{\partial C_{p}}{\partial \xi^{\prime}}=\frac{2 R e^{-1}}{h}\left[\frac{\partial^{2} u}{\partial \xi^{\prime 2}}+\frac{\partial^{2} u}{\partial \eta^{\prime 2}}-2 \frac{\xi^{\prime}}{h^{2}} \frac{\partial v}{\partial \eta^{\prime}}+2 \frac{\eta^{\prime}}{h^{2}} \frac{\partial v}{\partial \xi^{\prime}}\right] \\
& \frac{\partial C_{p}}{\partial \eta^{\prime}}=\frac{2 R e^{-1}}{h}\left[\frac{\partial^{2} v}{\partial \xi^{\prime 2}}+\frac{\partial^{2} v}{\partial \eta^{\prime 2}}+2 \frac{\xi^{\prime}}{h^{2}} \frac{\partial u}{\partial \eta^{\prime}}-2 \frac{\eta^{\prime}}{h^{2}} \frac{\partial u}{\partial \xi^{\prime}}\right]
\end{aligned}
$$

After this point, the Prandtl transposition (shearing transformation) is applied in order to suppress the perturbations on the parabola surface. As a result of this, the perturbation geometry is embedded in the wall boundary condition equations. The chain rules that are used for the application of the Prandtl transposition are: 


$$
\begin{array}{cc}
\frac{\partial}{\partial \xi^{\prime}}=\frac{\partial}{\partial \xi}-f_{\xi} \frac{\partial}{\partial \eta} & \frac{\partial}{\partial \eta^{\prime}}=\frac{\partial}{\partial \eta} \\
\frac{\partial^{2}}{\partial \xi^{\prime 2}}=\frac{\partial^{2}}{\partial \xi^{2}}-f_{\xi \xi} \frac{\partial}{\partial \eta}-2 f_{\xi} \frac{\partial^{2}}{\partial \xi \partial \eta}+f_{\xi}^{2} \frac{\partial^{2}}{\partial \eta^{2}} & \frac{\partial^{2}}{\partial \eta^{\prime 2}}=\frac{\partial^{2}}{\partial \eta^{2}}
\end{array}
$$

Making the relevant changes due to the Prandtl transposition, the gradient equations become,

$$
\begin{gathered}
\frac{\partial C_{p}}{\partial \xi}-f_{\xi} \frac{\partial C_{p}}{\partial \eta}=\frac{2 R e^{-1}}{h}\left[\frac{\partial^{2} u}{\partial \xi^{2}}-f_{\xi \xi} \frac{\partial u}{\partial \eta}-2 f_{\xi} \frac{\partial^{2} u}{\partial \xi \partial \eta}+\left(1+f_{\xi}^{2}\right) \frac{\partial^{2} u}{\partial \eta^{2}}-\frac{2 \xi}{h^{2}} \frac{\partial v}{\partial \eta}+\frac{2(\eta+f)}{h^{2}}\left(\frac{\partial v}{\partial \xi}-f_{\xi} \frac{\partial v}{\partial \eta}\right)\right] \\
\frac{\partial C_{p}}{\partial \eta}=\frac{2 R e^{-1}}{h}\left[\frac{\partial^{2} v}{\partial \xi^{2}}-f_{\xi \xi} \frac{\partial v}{\partial \eta}-2 f_{\xi} \frac{\partial^{2} v}{\partial \xi \partial \eta}+\left(1+f_{\xi}^{2}\right) \frac{\partial^{2} v}{\partial \eta^{2}}+2 \frac{\xi}{h^{2}} \frac{\partial u}{\partial \eta}-2 \frac{(\eta+f)}{h^{2}}\left(\frac{\partial u}{\partial \xi}-f_{\xi} \frac{\partial u}{\partial \eta}\right)\right]
\end{gathered}
$$

where $h=\sqrt{\xi^{2}+(\eta+f)^{2}}$. But the velocities along the surface are going to be zero due to no slip. Therefore, the corresponding derivatives of the velocities along the surface are also zero. So, $\frac{\partial \vec{V}}{\partial \xi}=0$ and $\frac{\partial^{2} \vec{V}}{\partial \xi^{2}}=0$. Inserting these into the gradient equations, it is seen that:

$$
\begin{gathered}
\frac{\partial C_{p}}{\partial \xi}-f_{\xi} \frac{\partial C_{p}}{\partial \eta}=\frac{2 R e^{-1}}{h}\left[-f_{\xi \xi} \frac{\partial u}{\partial \eta}-2 f_{\xi} \frac{\partial^{2} u}{\partial \xi \partial \eta}+\left(1+f_{\xi}^{2}\right) \frac{\partial^{2} u}{\partial \eta^{2}}-\frac{2 \xi}{h^{2}} \frac{\partial v}{\partial \eta}-\frac{2(\eta+f)}{h^{2}} f_{\xi} \frac{\partial v}{\partial \eta}\right] \\
\frac{\partial C_{p}}{\partial \eta}=\frac{2 R e^{-1}}{h}\left[-f_{\xi \xi} \frac{\partial v}{\partial \eta}-2 f_{\xi} \frac{\partial^{2} v}{\partial \xi \partial \eta}+\left(1+f_{\xi}^{2}\right) \frac{\partial^{2} v}{\partial \eta^{2}}+\frac{2}{h^{2}}\left(\xi+f_{\xi}(\eta+f)\right) \frac{\partial u}{\partial \eta}\right]
\end{gathered}
$$

Further combining the above equations, one gets an equation that only accounts for change of pressure along the wall:

$$
\frac{\partial C_{p}}{\partial \xi}=\frac{2 R e^{-1}}{h}\left\{\begin{array}{l}
\frac{\partial u}{\partial \eta}\left[-f_{\xi \xi}+\frac{2 f_{\xi} \xi}{h^{2}}+\frac{2 f_{\xi}^{2}(\eta+f)}{h^{2}}\right]-\frac{\partial v}{\partial \eta}\left[f_{\xi} f_{\xi \xi}+\frac{2 \xi}{h^{2}}+\frac{2 f_{\xi}(\eta+f)}{h^{2}}\right]+ \\
\left(1+f_{\xi}^{2}\right) \frac{\partial^{2} u}{\partial \eta^{2}}+\left(1+f_{\xi}^{2}\right) f_{\xi} \frac{\partial^{2} v}{\partial \eta^{2}}-2 f_{\xi} \frac{\partial^{2} u}{\partial \xi \partial \eta}-2 f_{\xi}^{2} \frac{\partial^{2} v}{\partial \xi \partial \eta}
\end{array}\right\}
$$

This final equation is a ready to use equation as a boundary condition. However, for the present study, the derivation is further continued to relate the velocity terms to the vorticity/stream function terms. To achieve this, no slip condition is inserted into the definitions of the velocity and vorticity terms. The velocity derivatives along the wall were also set to zero due to no slip condition. Based on these and 
using the definition of the stream function and vorticity the following are obtained. These analytic terms are to be inserted into the equation above which gives the pressure gradient along the wall.

$$
\begin{gathered}
\frac{\partial u}{\partial \eta}=-\frac{h \omega}{\left(1+f_{\xi}^{2}\right)} \\
\frac{\partial v}{\partial \eta}=-\frac{f_{\xi} h \omega}{\left(1+f_{\xi}^{2}\right)} \\
\frac{\partial^{2} u}{\partial \xi \partial \eta}=\frac{\psi_{\xi \eta \eta}}{h}+\frac{\omega\left[\xi+f_{\xi}(\eta+f)\right]}{h\left(1+f_{\xi}^{2}\right)} \\
\frac{\partial^{2} u}{\partial \eta^{2}}=\frac{2 \omega(\eta+f)}{h\left(1+f_{\xi}^{2}\right)}+\frac{\psi_{\eta \eta \eta}}{h} \\
\frac{\partial^{2} v}{\partial \eta^{2}}=-\frac{\left(\psi_{\xi \eta \eta}-f_{\xi} \psi_{\eta \eta \eta}\right)}{h}+\frac{f_{\xi} \omega\left[\xi+f_{\xi}(\eta+f)\right]}{h\left(1+f_{\xi}^{2}\right)}-\frac{f_{\xi \xi} h \omega}{\left(1+f_{\xi}^{2}\right)} \\
\psi_{\eta \eta \eta}=\frac{h^{2}}{\left(1+f_{\xi}^{2}\right)}\left[\frac{2 f_{\xi}}{h^{2}} \psi_{\xi \eta \eta}-\omega_{\eta}-\frac{2 \omega(\eta+f)}{h^{2}}-\frac{\omega f_{\xi \xi}}{\left(1+f_{\xi}^{2}\right)}\right] \\
\psi_{\xi \eta \eta}=\frac{h^{2}}{\left(1+f_{\xi}^{2}\right)}\left[\frac{2 f_{\xi} f_{\xi \xi} \omega}{\left(1+f_{\xi}^{2}\right)}-\omega_{\xi}-\frac{2 \omega\left(\xi+f_{\xi}(\eta+f)\right)}{h^{2}}\right]
\end{gathered}
$$




\section{APPENDIX D: VELOCITY AND ACCELERATION VECTORS}

The velocities are calculated in the transformed $\xi^{\prime}-\eta$ ' plane. Therefore, the velocity difference between the particle and the local flow is already calculated in the transformed plane. This means that the corresponding drag force can easily be decomposed into its components in the transformed plane. However, the force due to gravity and buoyancy are acting in the y direction. Therefore, these forces need to be expressed in terms of the transformed plane unit vectors. This is done as explained below.

The transformation function between the two planes is:

$$
x=\frac{\xi^{\prime 2}-\eta^{\prime 2}}{2} \text { and } y=\xi^{\prime} \eta^{\prime}
$$

Then, by making use of the differential geometry rules, the following are found:

$$
\hat{e}_{\xi^{\prime}}=\frac{x_{\xi^{\prime}}}{h} \hat{i}+\frac{y_{\xi^{\prime}}}{h} \hat{j} \text { and } \hat{e}_{\eta^{\prime}}=\frac{x_{\eta^{\prime}}}{h} \hat{i}+\frac{y_{\eta^{\prime}}}{h} \hat{j}, \text { where } h=\sqrt{\xi^{\prime 2}+\eta^{\prime 2}}
$$

Then,

$$
\hat{e}_{\xi^{\prime}}=\frac{\xi^{\prime}}{h} \hat{i}+\frac{\eta^{\prime}}{h} \hat{j} \text { and } \hat{e}_{\eta^{\prime}}=\frac{-\eta^{\prime}}{h} \hat{i}+\frac{\xi^{\prime}}{h} \hat{j}
$$

Therefore,

$$
v_{\xi^{\prime}}=\frac{\xi^{\prime}}{h^{2}} v_{x}+\frac{\eta^{\prime}}{h^{2}} v_{y} \text { and } v_{\eta^{\prime}}=\frac{-\eta^{\prime}}{h^{2}} v_{x}+\frac{\xi^{\prime}}{h^{2}} v_{y}
$$

where $v_{\xi^{\prime}}$ and $v_{\eta^{\prime}}$ are the velocity components in $\xi^{\prime}$ and $\eta^{\prime}$ directions, respectively. Also,

$$
v_{x}=\xi^{\prime} v_{\xi^{\prime}}-\eta^{\prime} v_{\eta^{\prime}} \text { and } v_{y}=\eta^{\prime} v_{\xi^{\prime}}+\xi^{\prime} v_{\eta^{\prime}}
$$

where $v_{x}$ and $v_{y}$ are the non-dimensional velocity components in $x$ and $y$ directions, respectively.

As far as the acceleration terms, the time derivative of the velocity terms given above are used. Therefore,

$$
a_{\xi^{\prime}}=\left[\xi^{\prime} a_{x}+\eta^{\prime} a_{y}-\xi\left(v_{\xi}^{2}-v_{\eta}^{2}\right)-2 \eta v_{\xi} v_{\eta}\right] / h^{2} \text { and, } a_{\eta^{\prime}}=\left[\xi^{\prime} a_{y}-\eta^{\prime} a_{x}+\eta\left(v_{\xi}^{2}-v_{\eta}^{2}\right)-2 \xi v_{\xi} v_{\eta}\right] / h^{2}
$$


where $a_{\xi^{\prime}}$ and $a_{\eta^{\prime}}$ are the acceleration components in $\xi^{\prime}$ and $\eta^{\prime}$ directions, respectively. Also, solving for the acceleration components in the physical $(x-y)$ plane:

$$
a_{x}=\xi^{\prime} a_{\xi^{\prime}}-\eta^{\prime} a_{\eta^{\prime}}+\left(v_{\xi^{\prime}}^{2}-v_{\eta^{\prime}}^{2}\right) \text { and } a_{y}=\eta^{\prime} a_{\xi^{\prime}}+\xi^{\prime} a_{\eta^{\prime}}+2 v_{\xi^{\prime}} v_{\eta^{\prime}}
$$

where $a_{x}$ and $a_{y}$ are the non-dimensional acceleration components in $x$ and $y$ directions, respectively.

The reference values that are used for non-dimensionalization are $V_{\text {inf }}$ for velocity, ler for length, $\operatorname{ler} / V_{\text {inf }}$ for time, and $V_{\text {inf }}^{2} /$ ler for acceleration. 


\title{
EGEMEN OL OGRETIM
}

\author{
Alley, Morgantown, West Virginia, 26501 \\ Phone: Home: (304) 291-6728 Work: (304) 293-3111x2466 \\ E-mail: eogretim@mix.wvu.edu
}

\section{EDUCATION}

- PhD in Aerospace Engineering, May 2006 (expected), West Virginia University, Morgantown, WV

- M.S. in Aerospace Engineering May 2002, West Virginia University, Morgantown, WV

- B.Sc. in Aeronautical Engineering, January 2000, Middle East Technical University, Ankara, Turkey

\section{RESEARCH EXPERIENCE}

May 2002 - January 2006: PhD research on "Investigation of relative humidity and induced-vortex effects on aircraft icing". The study involved both analytical and CFD investigations on the subject.

June 2001 - May 2002: Master's thesis on "Ice shape modeling enhancement for local flow NavierStokes". Use of the Fourier analysis was introduced as a method to model the ice shapes on the aircraft wing leading-edge.

Spring 2000: Study of an innovative compressor rotor blade. The goal of the study was to obtain a high mass flux and high compression rate by implementing centrifugal compression into an axial compressor.

Summer 1999: Participated as an intern in a Ph.D. research study at Von Karman Institute, Brussels, Belgium. The subject of the study was the CFD analysis of the air flow in turbines for the investigation of heat transfer phenomena related to flow ejection from the stator trailing edge.

Spring 1999: Developed an innovative design in the undergraduate helicopter design course: Replacement of the tail rotor by a vertical rear wing. Also, developed a new computational method and used it in the undergraduate aircraft engine design course project: Innovative use of cylindrical coordinates for potential flow solutions of compressor blades in cascade flow.

\section{TEACHING EXPERIENCE}

- Full instructor of the following courses, which includes the preparation of the syllabi, exams, quizzes, and projects, instruction of the course, and office hours:

- Fluid Mechanics at WVU, fall 2005

- Thermodynamics at WVU, spring 2004 and spring 2005

- Part time teaching assistant of a senior level CFD class at WVU

- Undergraduate research mentoring at WVU

- Graduate research mentoring at WVU

- One-year creativity education for gifted students as a volunteer teacher in Ankara, Turkey

- Curriculum development for the creativity improvement of gifted children in Ankara, Turkey

ACADEMIC JOURNAL PUBLICATIONS

- Ogretim, E., Huebsch, W.W., and Narramore, J. "Investigation of relative humidity and induced-vortex effects on aircraft icing" (will be submitted to J. of Aircraft for review)

- Ogretim, E. and Huebsch, W.W. "Pressure field around ice accreted airfoil leading-edge" (submitted to Int. J. Numer. Meth. Fluids for review)

- Huebsch, W. W., Moeykens, S., Yavuz, I. and Ogretim, E. "Multilevel implementation of FlowLab in Engineering Fluids" (submitted to Computers in Education Journal for review)

- Ogretim, E., Shinn, A. and Huebsch, W.W. "Ice accretion prediction based on neural networks", J. of Aircraft 2006; Vol. 43, No. 1, pp 233-240.

- Ogretim, E. and Huebsch, W.W., Narramore J., and Mullins, B. "Mechanisms for Downstream Ice Growth", 2005 SAE Transactions, Journal of Aerospace, 2006, pp. 1212-1219.

- Ogretim, E. and Huebsch, W.W. "A novel method for automated grid generation of ice shapes for localflow analysis”, Int. J. Numer. Meth. Fluids 2004; 44:579-597. 


\section{OTHER PUBLICATIONS}

- "Eye for an I: An Essay on Modeling of Nature and Life by Humans" (submitted to The Fountain for review)

- "One Day" (submitted to The Fountain for review)

- "Matchless Pearl" (in press; The Fountain)

- "Dynamic Ego", The Fountain, Vol. 53, Jan.-March 2006.

- "Resonant Egos and the Art of Married Life", The Fountain, Vol. 52, Oct.-Dec. 2005.

- "Adab: Education of one's ego - A Model for Gifted Education", The Fountain, Vol. 51, July-Sept. 2005.

\section{EDITORIAL EXPERIENCE}

- Single chapter review for Essentials of Fluid Mechanics, Cengel \& Cimbala, $1^{\text {st }}$ Edition, McGraw-Hill

- Review of articles for The Fountain magazine, 2005-present

\section{CONFERENCE PAPERS}

- Huebsch, W. W., Ogretim, E., Moeykens, S., and Yavuz, I. "Multilevel implementation of FlowLab in Engineering Fluids", AIAA paper 2006-286, Reno, NV, January, 2006.

- Ogretim, E., Huebsch, W.W., Narramore, J. and Mullins, B. "Mechanisms for downstream Ice Growth", SAE-WAC 2005-01-3375, Grapevine, TX, October 2005.

- Ogretim, E., Huebsch, W.W. and Shinn, A. "Ice accretion prediction based on neural networks", AIAA paper 2005-1245, Reno, NV, January, 2005.

- Ogretim, E. and Huebsch, W.W. "Effect of leading-edge ice roughness on downstream frost formation", AIAA paper 2004-0059, Reno, NV, January 2004

- Ogretim, E. and Huebsch, W.W. "Ice shape modeling enhancement for local flow Navier-Stokes", AIAA paper 2003-1072, Reno, NV, January 2003

\section{SEMINARS/PRESENTATIONS}

- Poster presentation on "Ice Accretion Prediction Based on Neural Networks", $1^{\text {st }}$ TASSA Annual Conference, Washington D.C., Feb. 2005

- Gave a seminar on giftedness and creativity at Ankara Science High School, Ankara, Turkey, 1999

\section{TECHNICAL SKILLS}

- Experienced in LINUX/UNIX and WINDOWS environments

- Proficient in FORTRAN

- Experience with TECPLOT, SMAGGICE, NEUROSHELL software

\section{RESEARCH INTERESTS}

- Fluid mechanics and Thermodynamics

- Aircraft icing

- Unsteady low-Reynolds number flows

- Computational Fluid Dynamics (CFD)

- Neural networks

- Theory of Relativity and Quantum Mechanics

\section{MEMBERSHIPS}

- AIAA: American Institute for Aerospace and Aeronautics

- TASSA: Turkish-American Scientists and Scholars Association

\section{SERVICE}

- President of the Turkish-American Dialogue Club, Aug. 2005 - May 2006

- President of the Turkish Student Association, April 2001 - Aug. 2003 


\section{OTHER WORK EXPERIENCES}

- Worked in Evansdale Dining Services, Morgantown, WV, from Oct. 2000 to Apr. 2001

\section{LANGUAGES}

- English at advanced level

- Turkish as native language

- French at intermediate level

\section{VOLUNTEER WORK}

- Carried out the "Turkey Talk" program at The Village at Heritage Point (a senior house in WV) as volunteer, fall 2003

- Visited several primary and secondary schools in West Virginia to make presentations about Turkey, its people and culture

- Represented Turkey at several international fair organizations

\section{REFERENCES}

\section{Dr. Gary J. Morris}

Prof. at West Virginia University

Mech. \& Aerospace Eng. Dept., Evansdale Dr., Morgantown, WV, 26506-6106

Ph: (304) 293-3111 x2342 Fax: (304) 293-8823

E-mail: gary.morris@mail.wvu.edu

\section{Dr. John L. Loth}

Prof. at West Virginia University

Mech. \& Aerospace Eng. Dept., Evansdale Dr., Morgantown, WV, 26506-6106

Ph: (304) 293-4111 x2343 Fax: (304) 293-8823

E-mail: john.loth@mail.wvu.edu

\section{Dr. John M. Kuhlman}

Prof. at West Virginia University

Mech. \& Aerospace Eng. Dept., Evansdale Dr., Morgantown, WV, 26506-6106

Ph: (304) 293-3111 x 2328 Fax: (304) 293-6689

E-mail: john.kuhlman@mail.wvu.edu

\section{Dr. Rakesh K. Gupta}

Berry Chair Prof. at West Virginia University

Chemical Engineering Dept., Evansdale Dr., Morgantown, WV, 26506-6102

Ph: (304) 293-2111 x2427 Fax: (304) 293-4139

E-mail: rakesh.gupta@mail.wvu.edu

\section{Dr. Wade W. Huebsch}

Asst. Prof. at West Virginia University

Mech. \& Aerospace Eng. Dept., Evansdale Dr., Morgantown, WV, 26506-6106

Ph: (304) 293-3111 x $2331 \quad$ Fax: (304) 293-6689

E-mail: wade.huebsch@mail.wvu.edu 